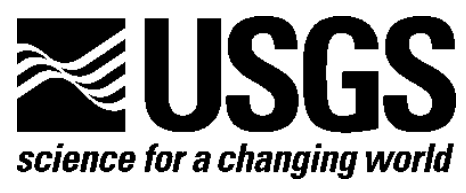

Prepared in cooperation with the Minnesota Pollution Control Agency

\title{
Endocrine Disrupting Chemicals in Minnesota Lakes-Water-Quality and Hydrological Data from 2008 and 2010
}

By Larry B. Barber, Jeffrey H. Writer, Steffanie H. Keefe, Greg K. Brown, Mark L. Ferrey, Nathan D. Jahns, Richard L. Kiesling, James R. Lundy, Beth H. Poganski, Donald O. Rosenberry, Howard E. Taylor, Olivia P. Woodruff, and Heiko L. Schoenfuss

Open-File Report 2012-1124

U.S. Department of the Interior U.S. Geological Survey 


\section{U.S. Department of the Interior \\ KEN SALAZAR, Secretary}

\section{U.S. Geological Survey \\ Marcia K. McNutt, Director}

U.S. Geological Survey, Reston, Virginia: 2012

For product and ordering information:

World Wide Web: http://www.usgs.gov/pubprod

Telephone: 1-888-ASK-USGS

For more information on the USGS-the Federal source for science about the Earth, its natural and living resources, natural hazards, and the environment: World Wide Web: http://www.usgs.gov Telephone: 1-888-ASK-USGS

Suggested citation:

Barber, L.B., Writer, J.H., Keefe, S.H., Brown, G.K., Ferrey, M.L., Jahns, N.D., Kiesling, R.L., Lundy, J.R., Poganski, B.H., Rosenberry, D.O., Taylor, H.E., Woodruff, O.P., and Schoenfuss, H. L., 2012, Endocrine disrupting chemicals in Minnesota lakes-Water-quality and hydrological data from 2008 and 2010: U.S. Geological Survey Open-File Report 2012-1124, 53 p.

Any use of trade, product, or firm names is for descriptive purposes only and does not imply endorsement by the U.S. Government.

Although this report is in the public domain, permission must be secured from the individual copyright owners to reproduce any copyrighted material contained within this report. 


\section{Contents}

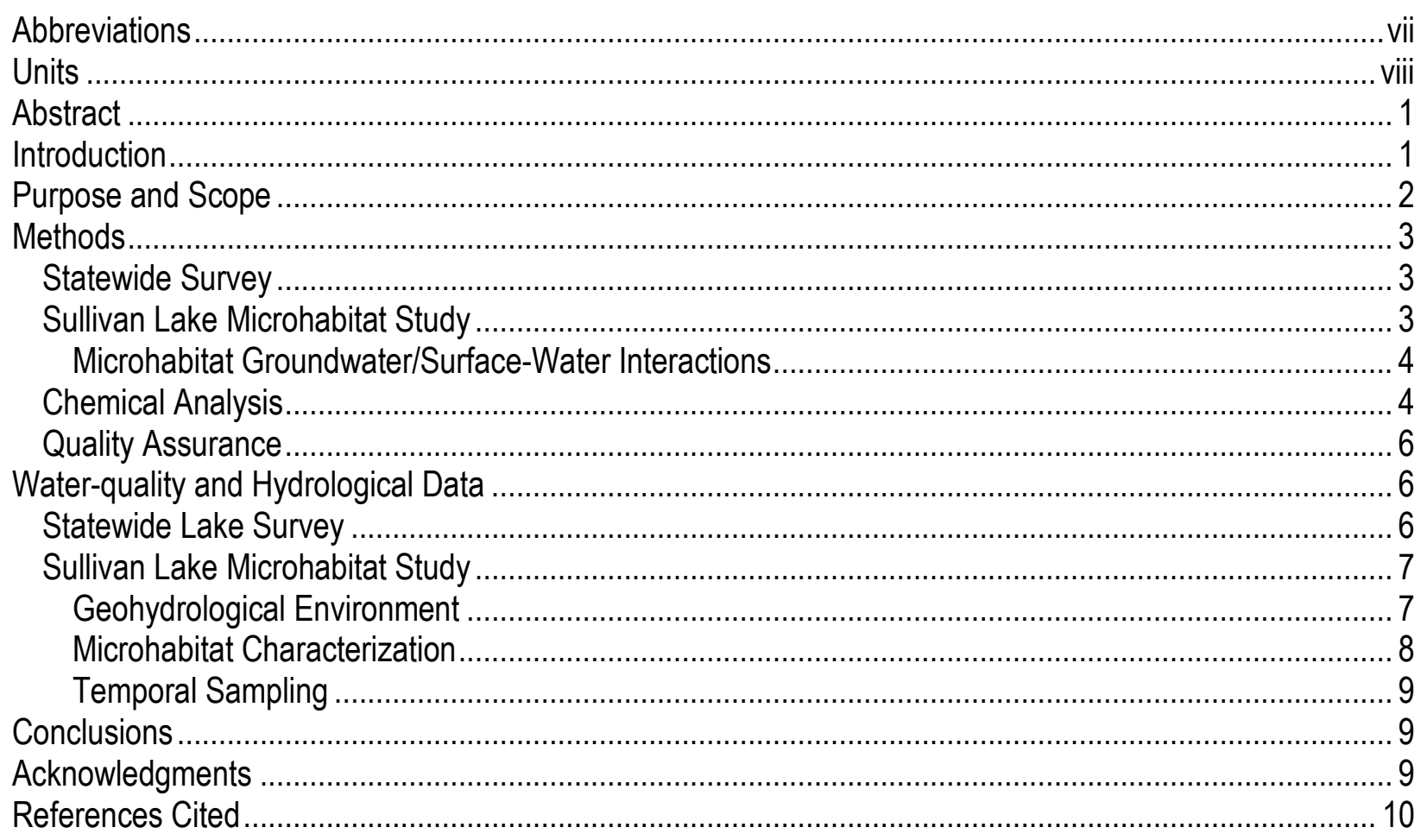

\section{Figures}

Figure 1. Site locations for the 14 Minnesota lakes sampled during 2008 and 2010 ......................... 14

Figure 2. Site locations for the four littoral-zone microhabitats investigated at Sullivan Lake, Minn........ 15

Figure 3. Site topography and preliminary hydraulic analysis of potential zones of groundwater discharge in Sullivan Lake, Minn 


\section{Tables}

Table 1. Site characteristics for the four Minnesota lakes that were sampled in this investigation .........17

Table 2. Trace elements and major elements measured by inductively coupled plasma/mass spectrometry (Garbarino and Taylor, 1995) and inductively coupled plasma/atomic-emission spectrometry (Garbarino and Taylor, 1979) at the U.S. Geological Survey National Research Program Laboratory, and the trace and major elements' approximate method detection limits (MDL)........19

Table 3. Acidic organic compounds measured by evaporation with derivatization and gas chromatography/mass spectrometry (Barber and others, 2000) at the U.S. Geological Survey National Research Program Laboratory

Table 4. Neutral organic compounds measured by continuous liquid-liquid extraction using methylene chloride with gas chromatography/mass spectrometry (Barber and others, 2000) at the U.S. Geological Survey National Research Program Laboratory.....

Table 5. Steroid and steroidal hormone compounds measured by octadecylsilica solid-phase extraction with derivatization and gas chromatography/tandem mass spectrometry (Barber and others, 2003; Foreman and others, 2011) at the U.S. Geological Survey National Research

Program Laboratory..... .22

Table 6. Summary of field measurements made at 11 Minnesota lakes during 2008 ...........................23

Table 7. Summary of major- and trace-element data for water samples collected from

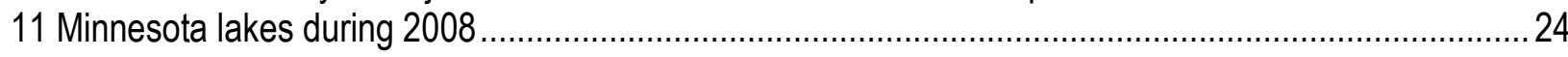

Table 8. Summary of acidic organic compound data for water samples collected from 11 Minnesota lakes during 2008

Table 9. Summary of neutral organic compound data for water samples collected from 11 Minnesota lakes during 2008.

Table 10. Summary of steroid and steroidal hormone compound data for water samples collected from 11 Minnesota lakes during 2008 30

Table 11. Summary of neutral organic compound data for sediment samples collected from 11 Minnesota lakes during 2008

Table 12. Summary of steroid and steroidal hormone compound data for sediment samples collected from 11 Minnesota lakes during 2008..

Table 13. Summary of neutral organic compound data for polar organic compound integrative samplers (POCIS) deployed in 11 Minnesota lakes during 2008.

Table 14. Summary of steroid and steroidal hormone compound data for passive organic compound integrative samplers (POCIS) deployed in 11 Minnesota lakes during 2008.....

Table 15. Summary of field measurements for water samples collected from four Minnesota lakes during 2010

Table 16. Summary of acidic organic compound data for water samples collected from four Minnesota lakes during 2010

Table 17. Summary of neutral organic compound data for water samples collected from four Minnesota lakes during 2010

Table 18. Summary of steroid and steroidal hormone compound data for water samples collected from four Minnesota lakes during 2010. 
Table 19. Summary of field data for preliminary microhabitat selection at Sullivan Lake, Minn., June 1, 2010 .

Table 20. Site characterization of Sullivan Lake microhabitat A (residential/septic influence) and water-quality values for pore-water samples collected July 8,2010

Table 21. Water column water-quality measurements for Sullivan Lake microhabitat A (residential/septic influence) collected from 1:30 to 3:00 PM on July 21, 2010

Table 22. Site characterization of Sullivan Lake microhabitat B (residential/septic influence) and water-quality values for pore-water samples collected July 8, 2010

Table 23. Water column water-quality measurements for Sullivan Lake microhabitat $B$ (residential/septic influence) collected 10:30 AM to 2:00 PM on July 21, 2010

Table 24. Site characterization of Sullivan Lake microhabitat C (stormwater/boat-ramp influence) and water-quality values for pore-water samples collected July 8, 2010

Table 25. Water column water-quality measurements for Sullivan Lake microhabitat $C$ (stormwater/boat ramp influence) collected 12:00 to 1:30 PM on July 21, 2010.

Table 26. Site characterization of Sullivan Lake microhabitat D (agricultural influence) and water-quality values for pore-water samples collected July 9, 2010

Table 27. Water column water-quality measurements for Sullivan Lake microhabitat $D$ (agricultural influence) collected 9:00 to 10:30 AM on July 21, 2010

Table 28. Summary of acidic organic compound data for water samples collected from four microhabitat sites in Sullivan Lake, Minn., on July 8, 2010.

Table 29. Summary of acidic organic compound data for water samples collected from four microhabitat sites in Sullivan Lake, Minn., on October 12, 2010

Table 30. Summary of neutral organic compound data for water samples collected from four microhabitat sites in Sullivan Lake, Minnesota on July 8, 2010

Table 31. Summary of neutral organic compound data for water samples collected from four microhabitat sites in Sullivan Lake, Minn., on October 12, 2010

Table 32. Summary of steroid and steroidal hormone compound data for water samples collected from four microhabitat sites in Sullivan Lake, Minn., on July 8, 2010.

Table 33. Summary of steroid and steroidal hormone compound data for water samples collected from four microhabitat sites in Sullivan Lake, Minn., on October 12, 2010 


\section{Conversion Factors}

Inch/Pound to SI

\begin{tabular}{lcl}
\hline \multicolumn{1}{c}{ Multiply } & By & To obtain \\
\hline centimeter $(\mathrm{cm})$ & Length & \\
meter $(\mathrm{m})$ & 0.3937 & inch (in) \\
kilometer $(\mathrm{km})$ & 3.281 & foot (ft) \\
\hline & 0.6214 & mile (mi) \\
\hline square kilometer $\left(\mathrm{km}^{2}\right)$ & Area & \\
\hline & 0.3861 & square mile (mi $\left.{ }^{2}\right)$ \\
\hline liter $(\mathrm{L})$ & Volume & gallon (gal) \\
\hline & 0.2642 & \\
\hline gram $(\mathrm{g})$ & Mass & ounce, avoirdupois (oz) \\
kilogram $(\mathrm{kg})$ & 0.03527 & pound, avoirdupois (lb) \\
\hline & 2.205 & \\
\hline torr & Pressure & pound per square inch (PSI) \\
\hline
\end{tabular}

Temperature in degrees Celsius $\left({ }^{\circ} \mathrm{C}\right)$ may be converted to degrees Fahrenheit $\left({ }^{\circ} \mathrm{F}\right)$ as follows: ${ }^{\circ} \mathrm{F}=\left(1.8 x^{\circ} \mathrm{C}\right)+32$

Vertical coordinate information is referenced to the North American Vertical Datum of 1988 (NAVD 88). Altitude, as used in this report, refers to distance above the vertical datum.

Specific conductance is given in microsiemens per centimeter at 25 degrees Celsius $\left(\mu \mathrm{S} / \mathrm{cm}\right.$ at $\left.25^{\circ} \mathrm{C}\right)$. Concentrations of chemical constituents in water are given either in milligrams per liter $(\mathrm{mg} / \mathrm{L})$ or micrograms per liter $(\mu \mathrm{g} / \mathrm{L})$. 


\section{Abbreviations}

$\begin{array}{ll}\text { AP, } & \text { alkylphenolethoxylates } \\ \text { SPE, } & \text { octadecylsilica solid-phase extraction } \\ \text { CASRN, } & \text { Chemical Abstract Services Registry Number } \\ \text { CLLE, } & \text { continuous liquid-liquid extraction } \\ \text { EVAP, } & \text { evaporated to dryness } \\ \text { GC/MS, } & \text { gas chromatography/mass spectrometry } \\ \text { GC/MS/MS, } & \text { gas chromatography/tandem mass spectrometry } \\ \text { LAS, } & \text { linear alkylbenzene sulfonate } \\ \text { MDL, } & \text { method detection limits } \\ \text { NA, } & \text { not available } \\ \text { NRPL, } & \text { National Research Program Laboratory } \\ \text { SIM, } & \text { selected ion monitoring } \\ \text { TM, } & \text { trademarked } \\ \text { USGS, } & \text { U.S. Geological Survey } \\ \text {--, } & \text { not determined }\end{array}$




\section{Units}

$\begin{array}{ll}\mathrm{cm}, & \text { centimeter } \\ { }^{\circ} \mathrm{C}, & \text { degree Celsius } \\ \mathrm{hr}, & \text { hour } \\ \mathrm{km}, & \text { kilometer } \\ \mathrm{km}{ }^{2}, & \text { square kilometer } \\ \mathrm{L}, & \text { liter } \\ \mathrm{m}, & \text { meter } \\ \mathrm{mg} / \mathrm{L}, & \text { milligram per liter } \\ \mathrm{mg} / \mathrm{L} \mathrm{C}, & \text { milligram per liter carbon } \\ \mathrm{mL}, & \text { milliliter } \\ \mathrm{mL} / \mathrm{min} / \mathrm{m}^{2}, & \text { milliliters per minute per square meter } \\ \mathrm{mm}, & \text { millimeter } \\ \mathrm{ng} / \mathrm{L}, & \text { nanogram per liter } \\ \mathrm{nm}, & \text { nanometer } \\ \mathrm{Std} \text { units, } & \text { standard pH units } \\ \mu \mathrm{g} / \mathrm{L}, & \text { microgram per liter } \\ \mu \mathrm{m}, & \text { micrometer } \\ \mu \mathrm{S} / \mathrm{cm}, & \text { microsiemens per centimeter } \\ \mathrm{v} / \mathrm{v}, & \text { volume/volume } \\ <, & \text { less than }\end{array}$




\title{
Endocrine Disrupting Chemicals in Minnesota Lakes-Water-Quality and Hydrological Data from 2008 and 2010
}

By Larry B. Barber, ${ }^{1}$ Jeffrey H. Writer, ${ }^{1}$ Steffanie H. Keefe, ${ }^{1}$ Greg K. Brown, ${ }^{1}$ Mark L. Ferrey, ${ }^{2}$

Nathan D. Jahns, ${ }^{3}$ Richard L. Kiesling, ${ }^{4}$ James R. Lundy, ${ }^{5}$ Beth H. Poganski, ${ }^{3}$ Donald O. Rosenberry, ${ }^{6}$

Howard E. Taylor, ${ }^{1}$ Olivia P. Woodruff, ${ }^{1}$ and Heiko L. Schoenfuss ${ }^{3}$

\begin{abstract}
Understanding the sources, fate, and effects of endocrine disrupting chemicals in aquatic ecosystems is important for water-resource management. This study was conducted during 2008 and 2010 to establish a framework for assessing endocrine disrupting chemicals, and involved a statewide survey of their occurrence in 14 Minnesota lakes and a targeted study of different microhabitats on a single lake. The lakes ranged in size from about 0.1 to 100 square kilometers, varied in trophic status from oligotrophic to eutrophic, and spanned a range of land-uses from wetlands and forest to agricultural and urban use. Water and sediment samples were collected from the near-shore littoral environment and analyzed for endocrine disrupting chemicals, including trace elements, acidic organic compounds, neutral organic compounds, and steroidal hormones. In addition, polar organic compound integrative samplers were deployed for 21 days and analyzed for the same organic compounds. One lake was selected for a detailed microhabitat study of multiple near-shore environments. This report compiles the results from the field measurements and laboratory chemical analysis of water, sediment, and polar organic compound integrative sampler samples collected during 2008 and 2010. Most of the organic compounds measured were not detected in any of the water samples, although a few compounds were detected in several of the lakes.
\end{abstract}

\section{Introduction}

Most research on the occurrence and effects of endocrine disrupting chemicals (EDCs) in the aquatic environment has focused on effluents from wastewater treatment plants (WWTPs) and effluent-impacted streams (Folmar and others, 1996; Jobling and others, 1998; Tyler and others, 1998; Barber and others, 2007; Vajda and others, 2008; Lee and others, 2010). However, a variety of natural and synthetic EDCs can enter lake environments via sources such as: runoff

\footnotetext{
${ }^{1}$ U.S. Geological Survey, 3215 Marine Street, Boulder CO 80303

${ }^{2}$ Minnesota Pollution Control Agency, 520 Lafayette Road, St. Paul, MN 55155

${ }^{3}$ St. Cloud State University, WSB-273, 720 Fourth Avenue South, St. Cloud, MN 56301

${ }^{4}$ U.S. Geological Survey, 2280 Woodale Drive, Mounds View, MN 55112

${ }^{5}$ Minnesota Department of Health, St. Paul, MN 55155

${ }^{6}$ U.S. Geological Survey, Denver Federal Center, Denver, CO 80225
} 
from surrounding urban and agricultural land use, septic system discharges from residences along the shoreline, and recreational activity (Swartz and others, 2006; Conn and others, 2006; Conn and others, 2010; Writer and others, 2010; Lee and others, 2011). In general, the most important estrogenic chemicals in the aquatic environment include the natural and synthetic steroidal hormones $17 \beta$-estradiol, estrone, and 17 $\alpha$-ethynylestradiol, and the alkyphenol compounds 4-nonylphenol and 4-tert-octylphenol (Van den Belt and others, 2004; Bistodeau and others, 2006; Sumpter and others, 2006; Barber and others, 2007; Schoenfuss and others, 2008).

Although the occurrence of EDCs in effluents and streams (and associated endocrine disruption in fish) has been documented, there is less information on the occurrence and impacts of EDCs in lakes that do not receive WWTP effluent discharges. For example, Lake Mead (Arizona and Nevada), which receives the discharge from a number of upstream WWTPs, has been shown to have elevated concentrations of synthetic chemicals and endocrine disruption in common carp (Rosen and others, 2006). The potential susceptibility of lake ecosystems to endocrine disruption has been documented by a rapid collapse in fish populations across multiple species after dosing a pristine Canadian lake for several years with approximately 5 nanogram per liter (ng/L) of the synthetic estrogen 17 $\alpha$-ethynylestradiol (Palace and others, 2002; Kidd and others, 2007). However, after the 17 $\alpha$-ethynylestradiol dosing was terminated, the fish populations recovered (Palace and others, 2009). Based partially on data presented in this report, Writer and others (2010) describe the low-level but widespread occurrence of EDCs and fish endocrine disruption in Minnesota lakes that do not have WWTP effluent inputs. Endocrine disruption also has been reported in fish from Lake Thun, Switzerland (Bogdal and others, 2009). Skelly and others (2010) have described endocrine disruption in frogs from urban ponds. These results suggest the widespread occurrence of EDCs and fish endocrine disruption in lakes that do not receive WWTP effluent discharges.

\section{Purpose and Scope}

The purpose of this cooperative study between the Minnesota Pollution Control Agency (MPCA) and the U.S. Geological Survey (USGS), which included collaboration with St.Cloud State University (SCSU), was to determine the occurrence of endocrine disrupting chemicals in water and sediment from lakes across the State of Minnesota. This report compiles data from a multi-tiered investigation into the occurrence of EDCs in Minnesota lakes conducted during 2008 and 2010 that consisted of: (1) a statewide survey of the chemistry of 14 lakes (11 sampled during 2008 and three sampled during 2010; two sampled both years), and (2) a detailed microhabitat study to characterize the hydrology and chemistry of the littoral zone in Sullivan Lake. Sullivan Lake is characteristic of many Minnesota lakes influenced by the surrounding landscape that includes agricultural land-use and residences using septic systems for onsite wastewater disposal. Results from the detailed study can be used to better understand the results from the statewide survey.

Companion investigations into endocrine-disruption in wild-caught fish and controlled field-exposure experiments using caged fathead minnows were conducted in conjunction with the water sampling in the 11 lakes sampled in 2008 (Ferrey and others, 2008) and in Sullivan Lake during 2010 (Poganski, 2011; Schoenfuss and Poganski, 2011). 


\section{Methods}

\section{Statewide Survey}

During 2008 and 2010, a statewide survey of 14 lakes representing a range of physiographic, geological, and land-use characteristics of Minnesota (fig. 1; table 1) was conducted to evaluate the occurrence of endocrine disrupting chemicals. The lakes covered a range of trophic status (oligotrophic to eutrophic) and anthropogenic inputs from agriculture and urban activities. Several sites were in remote locations with minimal development, including Northern Light Lake, Elk Lake, Beast Lake, Ryan Lake, and Bohall Lake. Between May 22 and August 3, 2008, water was collected during single sampling events from 11 lakes, starting in the southern part of the state and moving northward. Sediment samples from the same lakes were collected approximately one month later. Polar organic compound integrative samplers (POCIS; Alvarez and others, 2004) acquired from Environmental Sampling Technologies (St. Joseph, Missouri) were deployed during the summer of 2008 (at the time of water sampling) for 21 days at 11 of the lakes. During 2010, water samples were collected from three additional lakes and two of the lakes sampled in 2008 (Elk Lake and Sullivan Lake) were resampled.

Grab samples from the water surface were collected using a pre-cleaned stainless steel bucket rinsed with lake water and processed following USGS sampling protocols (U.S. Geological Survey, variously dated). Samples were transported on ice to the USGS National Research Program Laboratory (NRPL) in Boulder, Colorado where they were stored at 4 degrees Celsius $\left({ }^{\circ} \mathrm{C}\right)$ until analysis. Approximately 1 month after the water sampling, composite sediment samples were collected from the top 10 centimeters $(\mathrm{cm})$ directly beneath the bed surface in close proximity to the sites where water and POCIS samples were collected. The sediment samples were stored frozen until analysis. The POCIS samplers were attached at a depth of 1 meter $(\mathrm{m})$ to existing docks or to steel posts located approximately $5 \mathrm{~m}$ offshore. Upon retrieval, the POCIS samplers were placed in sealed cans and transported to the NRPL where they were stored at $4{ }^{\circ} \mathrm{C}$ until analysis. Water samplings and POCIS deployments were conducted at the same locations and during the same time periods as companion biological studies conducted by SCSU on endocrine disruption in wild-caught fish and caged fathead minnows (Ferrey and others, 2008).

\section{Sullivan Lake Microhabitat Study}

To provide a better understanding of the spatial and seasonal relationships between contaminant sources and processes occurring in the littoral zone, Sullivan Lake was selected for the detailed microhabitat study. Sullivan Lake has a relatively small watershed (3 square kilometers $\left(\mathrm{km}^{2}\right)$ ) with underlying glacial morphology that is characteristic of many Minnesota lakes. Sullivan Lake (fig. 2) is located near the town of St. Cloud in central Minnesota and is classified as meso-eutrophic (moderate biological productivity), has a surface area of 30 hectares (73 acres), and has a maximum depth of $16.5 \mathrm{~m}$ (Minnesota Department of Natural Resources, 2010). The bottom substrate varies from sand to detritus, but primarily is muck and silt. Predominant land use in the Sullivan Lake watershed was 49 percent cropland, 11 percent deciduous forests, 8 percent developed (residential), 7 percent wetland, and other land-use classifications were 5 percent or less (fig. 2; Minnesota Department of Natural Resources, 2010). The lake may potentially be influenced by residential land use (septic systems), recreational activities such (boating and fishing), and agricultural runoff. 
The near-shore environment of Sullivan Lake was evaluated at four microhabitat sites (fig. 2), including two residential influenced areas (sites A and B) with septic systems, a stormwater runoff and recreational activity influenced area (site $\mathrm{C}$ ), and an agricultural (animal and crop) runoff influenced area (site D) with no residential land use. Site C contained a boat ramp used by lake residents and the general public. Much of the shoreline near site $\mathrm{C}$ was covered by thick emergent wetland vegetation, and the bottom sediments consisted of detritus and organic muck. In contrast, lawns extended to the shorelines of the residential/septic sites (A and B) and the near-shore bottom sediments were generally sandy with little or no emergent vegetation. Surface-water samples were collected at each microhabitat site in pre-cleaned and baked amber glass bottles in the same manner as described for the statewide lake survey and shipped to the NRPL, where they were stored at $4^{\circ} \mathrm{C}$ until analysis. Summer and fall samplings were conducted in order to evaluate seasonal variability.

\section{Microhabitat Groundwater/Surface-Water Interactions}

The potential for groundwater/surface-water interactions was evaluated at the four microhabitat sites using near-shore transects for pore-water sampling (fig. 2) with ideal (site A) cross-section spacing of $1,5,10$, and $15 \mathrm{~m}$ perpendicular from shore and 1,3 , and $5 \mathrm{~m}$ parallel to shore. At site B, dense emergent wetland vegetation prevented sampling at two of the sites closest to shore. Sites $\mathrm{C}$ and $\mathrm{D}$ had dense emergent wetland vegetation that generally limited sampling the near-shore environment: two locations (1 near the shore and the other $15 \mathrm{~m}$ offshore) were sampled at site $\mathrm{C}$, and two transects were sampled at four locations at site D.

Vertical hydraulic-head gradient measurements and pore-water samples were obtained using a hydraulic potentiomanometer (Rosenberry and LaBaugh, 2008) and a retractable well screen inserted to a depth of $30 \mathrm{~cm}$ below the sediment/water interface. A peristaltic pump was used to draw water from the surrounding sediments at a rate of less than 10 milliliters $(\mathrm{mL})$ per minute (min) to minimize the hydrodynamic disturbance and avoid excessive degassing or inadvertent additions of lake water to the sample. A manometer was connected to the tubing from the well screen following collection of $60-\mathrm{mL}$ pore-water samples (the pumping period needed to eliminate air bubbles from the system) and measurements of hydraulic-head differences were made at each location. Sediments at sites $\mathrm{C}$ were too fine for pulling water through the well screen and precluded measurements of hydraulic gradients. Groundwater flux also was determined at site A using the seepage-meter techniques described in Rosenberry and LaBaugh (2008). Two seepage meters were installed at different points along the shoreline and two measurements were made for each meter.

Field measurements (including determination of temperature and lake depth) were made at the time of each sample collection. Water-column, water/sediment interface, and pore-water temperature were determined using a rigid probe with a thermistor thermometer at the end. Temperature was recorded at the water/sediment interface $\left(T_{\text {water/sediment }}\right)$ just above the bed surface and $30 \mathrm{~cm}$ below the water/sediment interface $\left(\mathrm{T}_{30} \mathrm{~cm}\right)$. In situ water-quality measurements (specific conductance, dissolved oxygen, and $\mathrm{pH}$ ) were taken with a field probe at various depths in the water column along a 5-point transverse transect parallel to the shoreline (fig. 2) at each microhabitat site.

\section{Chemical Analysis}

Field measurements, including temperature, $\mathrm{pH}$, specific conductance, and dissolved oxygen were made according to standard procedures (U.S. Geological Survey, variously dated). 
Dissolved (1-micrometer $(\mu \mathrm{m})$ glass fiber filter) organic carbon was analyzed by platinum catalyzed persulfate/ultraviolet light oxidation with infrared detection (Barber and others, 2001; Weishaar and others, 2003). Linear alkylbenzene sulfonate (LAS) and alkylphenolethoxylates (AP) were analyzed in unfiltered water samples by enzyme-linked immunosorbent assay (ELISA) using standard methods (http://www.abraxiskits.com). Trace and major elements (table 2) were determined in $0.45-\mu \mathrm{m}$ filtered water samples by inductively coupled plasma-mass spectrometry (Garbarino and Taylor, 1996; Taylor, 2001) and inductively coupled plasma-atomic emission spectrometry (Garbarino and Taylor, 1979). Because a stainless steel bucket was used in the collection of surface-water grab samples, there is the potential for the introduction of low concentrations of select trace metals and thus potential positive bias.

Unfiltered water samples were analyzed for organic constituents using three different analytical methods described in detail elsewhere (Barber and others, 2011). Acidic organic compounds (table 3) were evaporated to dryness (EVAP) and derivatized with 10 percent (v:v) acetyl chloride:propanol to form the propyl esters (Barber and others, 2000). Neutral organic compounds (table 4) were isolated using continuous liquid-liquid extraction (CLLE) with methylene chloride at $\mathrm{pH} 2$ following ionic strength adjustment (Barber and others, 2000).

Steroid and steroidal hormone compounds (table 5) were isolated by octadecyl silica solid-phase extraction (SPE) followed by elution with methanol, Florisil cleanup, and derivitization with Nmethyl-N-trimethylsilyltrifluoroacetamide (Barber and others, 2011; Foreman and others, 2012).

The CLLE and EVAP extracts were analyzed by electron impact gas chromatography/mass spectrometry (GC/MS) in both the full scan and selected ion monitoring (SIM) modes. The general chromatographic conditions were: Hewlett Packard (HP) 6890 GC; column - HP Ultra II ( 5 percent phenylmethyl silicone), $25 \mathrm{~m} \times 0.2$ millimeter $(\mathrm{mm}), 33-\mu \mathrm{m}$ film thickness; carrier gas, ultra high purity helium with a linear-flow velocity of $27 \mathrm{~cm}$ per second (sec); injection port temperature, $300^{\circ} \mathrm{C}$; initial oven temperature, $50^{\circ} \mathrm{C}$; split vent open, 0.75 min; ramp rate, $6^{\circ} \mathrm{C} / \mathrm{min}$ to $300^{\circ} \mathrm{C}$; hold time, $15 \mathrm{~min}$ at $300^{\circ} \mathrm{C}$. The mass spectrometer conditions were: HP 5973 Mass Selective Detector; tune with perflurotributylamine; ionization energy, $-70 \mathrm{eV}$; source pressure, $1 \times 10^{-5}$ torr; source temperature, $250^{\circ} \mathrm{C}$; interface temperature, $280^{\circ} \mathrm{C}$; full scan, 40 to 550 atomic mass units (amu) at $1 \mathrm{scan} / \mathrm{sec}$. Concentrations were calculated based on SIM data using diagnostic ions for each compound, which was identified based on matching of retention times (plus or minus $0.05 \mathrm{~min}$ ) and ion ratios (plus or minus 20 percent) determined from analysis of authentic standards. External calibration curves and internal standard procedures were used for calculating concentrations.

Gas chromatography/tandem mass spectrometry (GC/MS/MS) analysis of the derivatized SPE samples was conducted using a Waters QuattroMicro QqQ GC/MS/MS with an Agilent 6890 gas chromatograph. Chromatography was on a $30 \mathrm{~m} \times 0.25 \mathrm{~mm} \times 0.25 \mu \mathrm{m}$ Restek RtxXLB capillary column at a He flow rate of $1 \mathrm{~mL} / \mathrm{min}$ with the injection port maintained at $275^{\circ} \mathrm{C}$. The gas chromatograph was programmed on a variable temperature gradient from $100^{\circ} \mathrm{C}$ to $310^{\circ} \mathrm{C}$. For each compound, the most abundant ion in the electron impact spectrum was selected as a precursor, and appropriate conditions were selected to maximize signal for three precursor-product transitions per compound. Quantification was based on external calibration curves and internal standard procedure.

Target compounds in sediment were isolated by accelerated solvent extraction (ASE) with isopropyl alcohol and cleaned up on a Florosil column (Burkhardt and others, 2005). The sediment extracts were analyzed by GC/MS as for CLLE, and then derivitized and analyzed by $\mathrm{GC} / \mathrm{MS} / \mathrm{MS}$ as for SPE. The OASIS ${ }^{\mathrm{TM}}$ hydrophilic-lipophilic-balance SPE sequestration medium 
encased in the POCIS samplers was transferred into a glass column containing silanized glass wool and eluted with methanol. The methanol extracts were evaporated under nitrogen, augmented with dichloromethane:methanol (95:5 v:v), and cleaned up on Florosil column, followed by GC/MS analysis as for CLLE. The extract was then derivitized and analyzed by $\mathrm{GC} / \mathrm{MS} / \mathrm{MS}$.

\section{Quality Assurance}

The quality-assurance program consisted of field duplicates, field samples spiked with target compounds, field blanks, distilled water spiked with target compounds, and distilled water blanks. Target compounds spiked into the distilled water and natural water matrices were processed in the same manner as environmental samples and used to evaluate method recoveries. Recoveries for individual samples also were evaluated using surrogate standards spiked into each sample prior to isolation. No standard reference materials were available for the organic compounds evaluated in this study. For the EVAP and CLLE GC/MS, surrogate standards (tables 3 and 4) were added to the water samples prior to extraction and analysis and results are presented as percent recoveries. Target compound quantitation for the CLLE and EVAP GC/MS analysis used internal standards and multi-point calibration curves. Standards were obtained from Dr. Ehrenstorfer GmbH (Augsburg, Germany), Cambridge Isotope Laboratories (Cambridge, Massachusetts), Sigma-Aldrich (Milwaukee, Wisconsin), and Supelco (Bellefonte, Pennsylvania). Deuterated polynuclear aromatic hydrocarbon internal standards were added to the extracts prior to GC/MS analysis. The SPE GC/MS/MS analysis used 12 deuterated standards (table 5) added following sample collection and isotope dilution quantitation protocols (Foreman and others, 2012). The sediment and POCIS samples used the same surrogate standards and quantitation procedures as the CLLE and SPE methods.

Method detection limits (MDLs) for water samples were defined as the concentration equivalent to three times the mean detection value in method blanks or five times the baseline, whichever was greater. For the sediments, MDLs were determined to be three times the mean detection value in method blanks, corrected for sediment mass extracted. For the POCIS samples, MDLs were set at three times the instrument detection values, corrected for number of POCIS extracted. Spike and recovery studies were not done for the POCIS samplers.

\section{Water-quality and Hydrological Data}

\section{Statewide Lake Survey}

Results for the chemical analysis of water, sediment, and POCIS samples collected from 11 lake sites during 2008 are presented in tables 6 to 14. Results for the four lake sites sampled during 2010 are presented in tables 15 to 18 .

The field measurement (table 6) results for the 2008 sampling indicate that the chemical characteristics differed broadly among the lakes, as would be expected considering they ranged from oligotrophic to eutrophic. For example, specific conductance ranged from 33 microsiemens per centimeter $(\mu \mathrm{S} / \mathrm{cm})$ in oligotrophic Northern Light Lake to $580 \mu \mathrm{S} / \mathrm{cm}$ eutrophic Cedar Lake. Trace element results (table 7) varied. Concentrations of boron, which typically is associated with WWTP effluents (Barber and others, 2011) were relatively constant and ranged from $7 \mu \mathrm{g} / \mathrm{L}$ in Kabetogama Lake (oligotrophic) to $33 \mu \mathrm{g} / \mathrm{L}$ in Budd Lake (eutrophic). 
Organic compounds commonly associated with endocrine disruption (see tables 3 to 5) were detected in the water, sediment, and POCIS samples collected during 2008 (tables 8 to 14). Although only a fraction of the organic compounds measured in the water were detected and concentrations were typically at sub-microgram per liter $(\mu \mathrm{g} / \mathrm{L})$ levels, several compounds (including EDCs) had widespread occurrence. For example, ethylenediaminetetraacetic acid, 4-nonylphenol, and estrone were detected in the water of multiple lakes at concentrations ranging from 0.05 to $0.39,0.11$ to 0.21 , and 0.0006 to $0.0015 \mu \mathrm{g} / \mathrm{L}$, respectively. Most of the compounds detected in the water also were detected in the sediments, but at much higher concentrations. Likewise, most compounds detected in the water also were detected in the POCIS samples.

Table 15 summarizes field measurement data for the four lakes sampled during 2010. Ryan Lake and Beast Lake had similar water temperature, specific conductance, dissolved oxygen, and $\mathrm{pH}$ values. Bohall Lake had lower water temperature $\left(9.6^{\circ} \mathrm{C}\right)$ and higher dissolved oxygen (10.0 milligram per liter, $\mathrm{mg} / \mathrm{L})$ values. Elk Lake had the highest water temperature $\left(11.8^{\circ} \mathrm{C}\right)$ and specific conductance $(294$ micro Siemens per centimeter, $\mu \mathrm{S} / \mathrm{cm})$ values, and the lowest dissolved oxygen $(8.6 \mathrm{mg} / \mathrm{L})$.

Table 16 summarized the acidic organic compounds detected in the lake water samples collected during 2010. Ethylenediaminetetraacetic acid (EDTA) was detected in Elk Lake $(0.53 \mu \mathrm{g} / \mathrm{L})$, Ryan Lake $(0.40 \mu \mathrm{g} / \mathrm{L})$, and Bohall Lake $(0.06 \mu \mathrm{g} / \mathrm{L})$, but nitrilotriacetic acid (NTA) and 4-nonylphenolethoxycarboxylates (4-NPEC) were not detected in any of the lakes. Surrogate standard recoveries ranged from 54 to 67 percent. Neutral organic compounds are summarized in table 17. Trace levels of 4-methylphenol; 2,6-di-tert-butyl-1,4-benzoquinone; and 2,6-di-tertbutyl-4-methylphenol were detected in several lakes. Surrogate standard recoveries ranged from less than 10 to 85 percent. Steroid and steroidal hormone compound data from lake samples collected during 2010 are summarized in table 18. Cholesterol, coprostanol, and estrone were detected in the lakes. Surrogate standard recoveries ranged from 37 to 115 percent.

\section{Sullivan Lake Microhabitat Study}

\section{Geohydrological Environment}

Analyses of topographic maps, aerial photographs, and field surveys of Sullivan Lake were used to provide preliminary information on potential groundwater/surface-water interactions (Winter, 1998). The landscape around Sullivan Lake is characterized by Pleistocene glacial deposits and appears to have been part of a larger lake (perhaps connecting to Maple Lake; fig. 2) that during glacial times was dammed up behind the 50-foot high, esker-like ridge along the eastern shoreline (fig. 3). The lake stage may have continued to rise until the lake eventually eroded through the ridge creating the narrow, deeply-incised canal-like outlet near the southeast corner of Sullivan Lake. The presence of wetlands adjacent to the north, northwest, and southwest shorelines indicates that hydraulically driven groundwater discharge likely is shifted to the margins between the uplands and wetlands rather than occurring at the boundary between the wetlands and the open-water lake or could be controlled by lake level in topographically low areas. The predominant geologic feature, from the perspective of groundwater discharge into the lake, is the esker-like ridge along the eastern and southeastern shoreline. Higher groundwater head beneath the ridge creates a hydraulic gradient that increases the potential for groundwater discharge along the western shoreline, which coincides with the areas of maximum residential density and creates the greatest potential for onsite wastewater-disposal systems to discharge into 
the lake. Storm water and agricultural runoff along the western shoreline is potentially buffered by the wetlands, which may attenuate chemicals before they enter the lake.

\section{Microhabitat Characterization}

A preliminary shoreline site investigation was conducted during the microhabitat siteselection process and the results are presented in table 19. Based on these results and hydrological considerations, four sampling sites were selected. Microhabitat A (residential/septic influence) had several hydraulic head values that were higher than the lake surface (positive values, table 20) and that ranged up to $15 \mathrm{~mm}$. The hydraulic-head values were generally low near the shore and greatest at sample location (point 1,3). The near-shore seepage-meter measurements indicated groundwater influx of -0.94 to 4.3 milliliters per minute per square meter $\left(\mathrm{mL} / \mathrm{min} / \mathrm{m}^{2}\right)$. Dissolved organic carbon values in the pore water samples ranged from 2.1 to $7.4 \mathrm{mg} / \mathrm{L}$. Linear alkylbenzene sulfonate values in pore water samples ranged from less than 0.02 to $0.92 \mathrm{mg} / \mathrm{L}$. There was only a single AP detection $(0.14 \mathrm{mg} / \mathrm{L}) . \mathrm{T}_{30 \mathrm{~cm}}$ measurements were

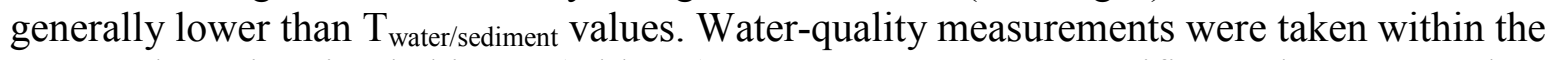
water column in microhabitat A (table 21). Water temperature, specific conductance, and $\mathrm{pH}$ were generally constant with depth with the exception of the 15, 5 point. Dissolved oxygen values were either constant within the water column or decreased with depth.

Microhabitat B (residential/septic influence) had several hydraulic-head values that were slightly lower than the lake surface (negative values, table 22), suggesting the potential for flow from the lake to groundwater at this location. Dissolved organic carbon values in the pore-water samples from microhabitat B $(7.6$ to $11.4 \mathrm{mg} / \mathrm{L})$ were greater than microhabitat A. The LAS values ranged from less than 0.02 to $0.12 \mathrm{mg} / \mathrm{L}$, and there were sporadic detections of AP (up to $0.11 \mathrm{mg} / \mathrm{L})$. No $\mathrm{T}_{30 \mathrm{~cm}}$ or $\mathrm{T}_{\text {water/sediment }}$ measurements were recorded in microhabitat $\mathrm{B}$. The watercolumn temperature, specific conductance, dissolved oxygen, and $\mathrm{pH}$ were relatively constant at microhabitat B (table 23).

Dense emergent vegetation limited access to sampling locations in microhabitat $\mathrm{C}$ (stormwater/boat ramp influence). Dissolved organic carbon values of pore-water samples ranged from 7.3 to $11.2 \mathrm{mg} / \mathrm{L}$ (table 24$)$. Low levels of LAS $(0.03 \mathrm{mg} / \mathrm{L})$ and AP $(0.11 \mathrm{mg} / \mathrm{L})$ were detected in pore-water samples. The $\mathrm{T}_{30 \mathrm{~cm}}$ temperatures $\left(17.7\right.$ to $\left.19.4^{\circ} \mathrm{C}\right)$ were lower than the $\mathrm{T}_{\text {water/sediment }}$ temperatures $\left(23.7\right.$ to $\left.28.6^{\circ} \mathrm{C}\right)$. A thick layer of organic muck at the water/sediment interface prevented hydraulic head measurements. Measurements taken within the water column in microhabitat $\mathrm{C}$ indicated that water temperature and specific conductance were relatively constant (table 25). Dissolved oxygen concentrations decreased with depth in the water column and near-bottom values were as much as 43 percent lower than near-surface values. The 3,5 point was an exception where dissolved oxygen and $\mathrm{pH}$ were fairly constant with depth.

Dense emergent vegetation and deep water limited sampling in microhabitat D (agricultural influence). A thick layer of organic muck near the water/sediment interface limited hydraulic head measurements (table 26). Linear alkylbenzene sulfonates were detected at concentrations ranging from 0.02 to $0.11 \mathrm{mg} / \mathrm{L}$, and no AP were detected. Values for $\mathrm{T}_{30 \mathrm{~cm}}$ $\left(16.7\right.$ to $\left.18.8^{\circ} \mathrm{C}\right)$ were lower than $\mathrm{T}_{\text {water/sediment }}\left(19.9\right.$ to $\left.23.8^{\circ} \mathrm{C}\right)$. Measurements taken within the water column at microhabitat $\mathrm{D}$ (table 27 ) indicated that temperature was relatively constant and dissolved oxygen and $\mathrm{pH}$ decreased with depth (with the exception of 0,5 where they remained relatively constant). Specific conductance values did not follow the same trends. 


\section{Temporal Sampling}

Acidic organic compounds detected in the summer 2010 Sullivan Lake microhabitat sampling are listed in table 28. Ethylenediaminetetraacetic acid, nitrilotriacetic acid, and 4-nonylphenolmonoethoxycarboxylic acid were detected at all of the microhabitat sites. Surrogate standard recoveries ranged from 66 to 98 percent. Acidic organic compounds detected in the fall 2010 sampling are listed in table 29. Ethylenediaminetetraacetic acid was present in all microhabitats, and microhabitat A (residential/septic influence) had the only detection of nitrilotriacetic acid and 4-nonylphenoldiethoxycarboxylic acid. Surrogate standard recoveries ranged from 46 to 70 percent.

Neutral organic compounds detected in the summer 2010 Sullivan Lake microhabitat sampling are listed in table 30. Several compounds were detected at multiple sites, including bisphenol A; 2,6-di-tert-butyl-1,4-benzoquinone; 2,6-di-tert-butyl-4-methylphenol; N,N-diethylmeta-toluamide; 4-methylphenol; and 4-nonylphenol. Microhabitat A had the highest levels of N,N-diethyl-m-toluamide and 4-nonylphenol, and had the only caffeine detection among the four sites. Surrogate standard recoveries ranged from 29 to 99 percent, and matrix spike recoveries ranged from less than 10 to 163 percent. Neutral organic compounds detected in the fall 2010 sampling are listed in table 31. Sites A and B had detections of bisphenol A; 2,6-di-tert-butyl1,4-benzoquinone; 2,6-di-tert-butyl-4-methylphenol; N,N-diethyl-meta-toluamide; and 4-nonylphenol. 4-tert-Octylphenol and caffeine also were detected at sites A and B. Surrogate recoveries ranged from 19 to 57 percent.

Steroid and steroidal hormone analysis for the summer 2010 Sullivan Lake microhabitat sampling are listed in table 32. Cholesterol, coprostanol, and estrone were detected at all microhabitat sites and 4-androstene-3,17-dione was detected at all sites except microhabitat A. Surrogate standard recoveries ranged from less than 10 to 183 percent, and matrix spike recoveries (excluding cholesterol, which had such high background concentrations it was difficult to calculate recoveries) ranged from 15 to 235 percent. Table 33 lists steroid and steroidal hormone analysis of surface-water samples from each microhabitat site collected in the fall of 2010. Cholesterol and coprostanol were detected at all sites, and a single detection of estrone occurred in microhabitat D. Surrogate standard recoveries ranged from 15 to 121 percent.

\section{Conclusions}

This report presents the results from field measurements and laboratory analysis for water, sediment, and POCIS samples collected from 14 Minnesota lakes during 2008 and 2010. These data provide a snapshot of the occurrence of a variety of potential endocrine-disrupting compounds in the near shore environment of 14 geographically distributed Minnesota lakes covering a range of land use and trophic status. One of the lakes, Sullivan Lake, was studied in detail to assess potential contaminant sources and evaluate the spatial and seasonal relationships.

\section{Acknowledgments}

The field assistance of Harold Wiegner (Minnesota Pollution Control Agency) is greatly appreciated. We also thank the homeowners on Sullivan Lake for access to their properties. This study was supported by the U.S. Geological Survey's National Research Program and Toxics Substances Hydrology Program. 


\section{References Cited}

Alvarez, D.A., Petty, J.D., Huckins, J.N., Jones-Lepp, T.L., Getting, D.T., Goddard, J.P., and Manahan, S.E., 2004, Development of a passive, in situ, integrative sampler for hydrophilic organic contaminants in aquatic environments: Environmental Toxicology and Chemistry, v. 23, p. 1640-1648.

Barber, L.B., Brown, G.K., and Zaugg, S.D., 2000, Potential endocrine disrupting organic chemicals in treated municipal wastewater and river water, in Analysis of environmental endocrine disruptors. Keith, L.H., Jones-Lepp, T.L., and Needham, L.L., eds.: Washington D.C., American Chemical Society Symposium Series 747, p. 97-123.

Barber, L.B., Leenheer, J.A., Noyes, T.I., and Stiles, E.A., 2001, Transformation of dissolved organic matter in treatment wetlands: Environmental Science and Technology, v. 35, p. 4805-4816.

Barber, L.B., Lee, K.E., Swackhamer, D.L., and Schoenfuss, H.L., 2007, Reproductive responses of male fathead minnows exposed to wastewater treatment plant effluent, effluent treated with XAD8 resin, and an environmentally relevant mixture of alkylphenol compounds: Aquatic Toxicology, v. 82, p. 36-47.

Barber, L.B., Antweiler, R.C., Flynn, J.L., Keefe, S.H., Kolpin, D.W., Roth, D.A., Schnoebelen, D.J., Taylor, H.E., and Verplanck, P.L., 2011, Lagrangian mass-flow investigations of inorganic contaminants in wastewater-impacted streams: Environmental Science and Technology, v. 45, p. 2575-2583.

Barber, L.B., Keefe, S.H., Kolpin, D.W., Schnoebelen, D.J., Flynn, J.L., Brown, G.K., Furlong, E.T., Gray, J.L., Glassmeyer, S.T., Meyer, M.T., Sandstrom, M.W., Taylor, H.E., and Zaugg, S.D., 2011, Lagrangian sampling of wastewater treatment plant discharges into Boulder Creek, Colorado and Fourmile Creek, Iowa during the summer of 2003 and spring of 2005Hydrological and water-quality data: U.S. Geological Survey Open-File Report 2011-1054. (Also available at http://pubs.usgs.gov/of/2011/1054/.)

Bistodeau, T.J., Barber, L.B., Bartell, S.E., Cediel, R.A., Grove, K.J., Klaustermeier, J., Woodard, J.C., Lee, K.E., and Schoenfuss, H.L., 2006. Larval exposure to environmentally relevant mixtures of alkylphenolethoxylates reduces reproductive competence in male fathead minnows: Aquatic Toxicology, v. 79, p. 268-277.

Bogdal, C., Naef, M., Schmid, P., Kohler, M., Zennegg, B.D., Scheringer, M., and Hungerbuhler, K., 2009, Unexplained gonad alterations in whitefish (Coregonus spp.) from Lake Thun, Switzerland: Levels of persistent organic pollutants in different morphs. Chemosphere, v. 74, p. 434-440.

Burkhardt, M.R., ReVello, R.C., Smith, S.G., and Zaugg, S.D., 2005, Pressurized liquid extraction using water/isopropanol coupled with solid-phase extraction cleanup for industrial and anthropogenic waste-indicator compounds in sediment: Analytica Chimica Acta, v. 534, p. 89-100.

Conn, K.E., Barber, L.B., Brown, G.K., and Siegrist, R.L., 2006, Occurrence and fate of organic contaminants during onsite wastewater treatment: Environmental Science and Technology, v. 40, p. 7358-7366.

Conn, K.E., Siegrist, R.L., Barber, L.B., and Meyer, M.T., 2010, Fate of trace organic compounds during vadose zone soil treatment in an onsite wastewater system: Environmental Toxicology and Chemistry, v. 29, p. 285-293. 
Ferrey, M., Preimesberger, A., Schoenfuss, H.L., Kiesling, R., Barber, L.B., Writer, J., 2008, Statewide endocrine disrupting compound monitoring study, 2007-2008: Minnesota Pollution Control Agency, Legislative Report tdr-g1-08, St. Paul, Minn., 87 p.

Folmar, L.C., Denslow, N.D., Rao, V., Chow, M., Crain, D.A., Enblom, J., Marcino, J., Guillette, L.J., Jr., 1996, Vitellogenin induction and reduced serum testosterone concentrations in feral male carp (Cyprinus carpio) captured near a major metropolitan sewage treatment plant: Environmental Health Perspectives, v. 104, p. 1096-1101.

Foreman, W.T., Gray, J.L., ReVello, R.C., Lindley, C.F., Losche, S.A., and Barber, L.B., 2012, Determination of steroid hormones and related compounds in filtered and unfiltered water by solid-phase-extraction, derivatization and gas chromatography with tandem mass spectrometry: U.S. Geological Survey Techniques and Methods, book 5, sec. B, chap. 9.

Garbarino, J.R. and Taylor, H.E., 1979, An inductively coupled plasma-atomic emission spectrometric method for routine water quality testing: Applied Spectroscopy, v. 33, p. 220-226.

Garbarino, J.R. and Taylor, H.E., 1996, Inductively coupled plasma-mass spectrometric method for the determination of dissolved trace elements in natural water: U.S. Geological Survey Open-File Report 94-358, 49 p.

Jobling, S., Nolan, M., Tyler, C.R., Brighty, G., and Sumpter, J.P., 1998, Widespread sexual disruption in wild fish: Environmental Science and Technology, v. 32, p. 2498-2506.

Kidd, K.A., Blanchfield, P.J., Mills, K.H., Palace, V.P., Evans, R.E., Lazorchak, J.M., and Flick, R.W., 2007, Collapse of a fish population following exposure to a synthetic estrogen:

Proceedings of the National Academy of Sciences of the United States of America, v. 104, p. 8897-8901.

Lee, K.E., Schoenfuss, H.L., Barber, L.B., Writer, J.H., Blazer, V.B., Kiesling, R.L., and Ferrey, M.L., 2010, Endocrine active chemicals and endocrine disruption in Minnesota streams and lakes-Implications for aquatic resources 1994-2009: U.S. Geological Survey Scientific Investigations Report 2010-5107, 47 p. with Appendixes. (Also available at http://pubs.usgs.gov/sir/2010/5107/.)

Lee, K.E., Langer, S.K., Barber, L.B., Writer, J.H., Ferrey, M.L., Schoenfuss, H.L., Furlong, E.T., Foreman, W.T., Gray, J.L., ReVello, R.C., Martinovic, D., Woodruff, O.P., Keefe, S.H., Brown, G.K., Taylor, H.E., Ferrer, I., and Thurman, E.M., 2011, Endocrine active chemicals, pharmaceuticals, and other chemicals of concern in surface water, wastewater-treatment plant effluent, and bed sediment, and biological characteristics in selected streams, MinnesotaDesign, methods, and data, 2009: U.S. Geological Survey Data Series 575, 48 p. plus Appendixes. (Also available at http://pubs.usgs.gov/ds/575/.)

Minnesota Department of Natural Resources, 2010, LakeFinder: St. Paul, Minn., Minnesota Department of Natural Resources, accessed March 15, 2010, at http://www.dnr.state.mn.us/lakefind.index.html.

Palace, V.P., Evans, R.E., Wautier, Kerry, Baron, Christopher, Vandenbyllardt, Lenore, Vandersteen, Wendy, and Kidd, Karen, 2002, Induction of vitellogenin and histological effects in wild fathead minnows from a lake experimentally treated with the synthetic estrogen, ethynylestradiol: Water Quality Research Journal of Canada, v. 37, p. 637-650.

Palace, V.P., Evans, R.E., Wautier, K.G., Mills, K.H., Blanchfield, P.J., Park, B.J., Baron, C.L., and Kidd, K.A, 2009, Interspecies differences in biochemical, histopathological, and population responses in four wild fish species exposed to ethynylestradiol added to a whole lake: Canadian Journal of Fish and Aquatic Science, v. 66, p. 1920-1935. 
Poganski, B.H., 2011, Assessment of microhabitat at differences on biological effects of Bluegill Sunfish in lake environments: St. Cloud., Minn., Saint Cloud State University, MS Thesis, $96 \mathrm{p}$.

Rosen, M.R., Goodbred, S.L., Patiño, R., Leiker, T.J., and Orsak, E., 2006, Investigations of the effects of synthetic chemicals on the endocrine system of common carp in Lake Mead, Nevada and Arizona: U.S. Geological Survey Fact Sheet 2006-3131, 4 p. (Also available at http://pubs.usgs.gov/fs/2006/3131/.)

Rosenberry, D.O. and LaBaugh, J.W., 2008, Field techniques for estimating water fluxes between surface water and ground water: U.S. Geological Survey, Techniques and Methods 4-D2, 128 p. (Also available at http://pubs.usgs.gov/tm/04d02/.)

Schoenfuss, H.L. and Poganski, B., 2011, Assessing the contribution of microhabitat differences on the biological effects of endocrine active compounds in bluegill sunfish in Sullivan Lake, MN: Minnesota Pollution Control Agency, Legislative Report tdr-g1-08c, St. Paul, Minn., $16 \mathrm{p}$.

Schoenfuss, H.L., Bartell, S.E., Bistodeau, T.B., Cediel, R.A., Grove, K.J., Zintek, L., Lee, K.E., and Barber, L.B., 2008, Impairment of the reproductive potential of male fathead minnows by environmentally relevant exposures to 4-nonylphenol: Aquatic Toxicology, v. 86, p. 91-98.

Skelly, D.K., Bolden, S.R., and Dion, K.B., 2010, Intersex frogs concentrated in suburban and urban landscapes: EcoHealth, DOI: 10.1007/s10393-010-0348-4, 6 p.

Sumpter, J.P., Johnson, A.C., Williams, R.J., Kortenkamp, A., and Scholze, M., 2006, Modeling effects of mixtures of endocrine disrupting chemicals at the river catchment scale:

Environmental Science and Technology, v. 40, p. 5478-5489.

Swartz, C.H., Reddy, S., Benotti, M.J., Yin, H., Barber, L.B., Brownawell, B.J., and Rudel, R., 2006, Steroid estrogens, nonylphenol ethoxylate metabolites, and other wastewater contaminants in groundwater affected by a residential septic system on Cape Cod, MA: Environmental Science and Technology, v. 40, p. 4894-4902.

Taylor, H.E., 2001, Inductively coupled plasma-mass spectrometry-Practices and techniques: San Diego, Calif., Academic Press, 104 p.

Tyler, C.R., Jobling, S., and Sumpter, J.P., 1998, Endocrine disruption in wildlife-A critical review of the evidence: Critical Reviews in Toxicology, v. 28, p. 319-361.

U.S. Geological Survey, variously dated, National field manual for the collection of waterquality data: U.S. Geological Survey Techniques of Water Resources Investigations, Book 9, Chapter A1-A9, available online at http://pubs.water.usgs.gov/twri9A.

U.S. Geological Survey, 2010, The USGS Land Cover Institute, accessed 2010 at http://landcover.usgs.gov/.

Vajda, A.M., Barber, L.B., Gray, J.L., Lopez, E.M., Woodling, J.D., and Norris, D.O., 2008, Reproductive disruption in fish downstream from an estrogenic wastewater effluent: Environmental Science and Technology, v. 42, p. 3407-3414.

Van den Belt, K., Berckmans, P., Vangenechten, C., Verheyen, R., and Witters, H., 2004, Comparative study on the in vitro/in vivo estrogenic potencies of $17 \beta$-estradiol, estrone, 17 $\alpha$-ethinyl estradiol, and nonylphenol: Aquatic Toxicology, v. 66, p. 183-195.

Weirshaar, J. L., Aiken, G. R., Bergamaschi, B.A., Fram, M.S., Fujii, R., and Mopper, K., 2003, Evaluation of specific ultraviolet absorbance as an indicator of the chemical composition and reactivity of dissolved organic carbo: Environmental Science and Technology, v. 37, p. 4702-4708. 
Winter, T.C., 1998, Relation of streams, lakes, and wetlands to groundwater flow systems: Hydrogeology Journal, v. 7, p. 28-45.

Writer, J.H., Barber, L.B., Brown, G.K., Taylor, H.E., Kiesling, R.L., Ferrey, M.L., Jahns, N.D., Bartell, S. E., and Schoenfuss, H. L., 2010, Anthropogenic tracers, endocrine disrupting chemicals, and endocrine disruption in Minnesota lakes. Science of the Total Environment, v. 409 , p. $100-111$. 


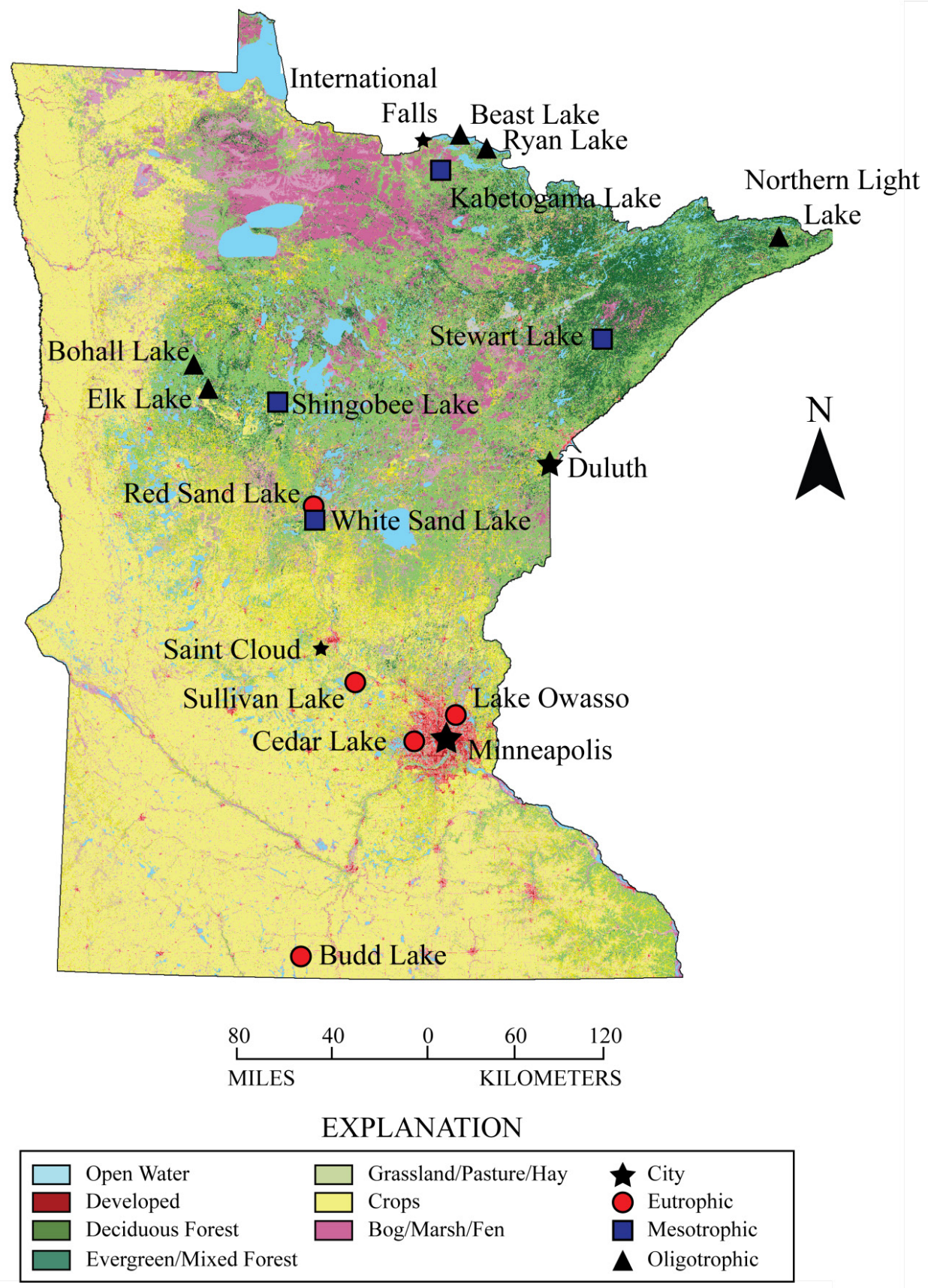

Base from Minnesota Department of Natural Resources, 1:24,000 Universal Transverse Mercator projection, Zone 15

Figure 1. Site locations for the 14 Minnesota lakes sampled during 2008 and 2010 (after Writer and others, 2010). 


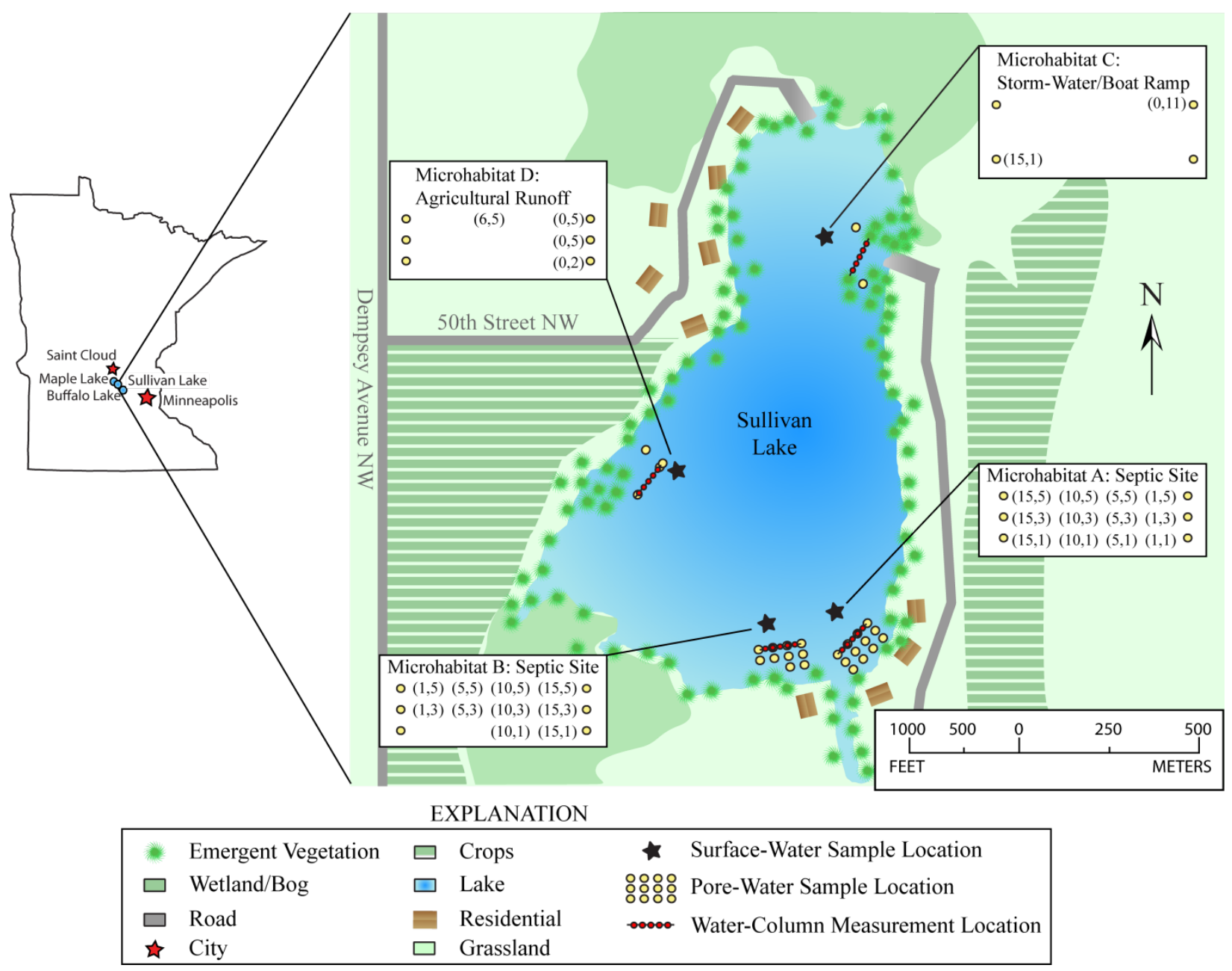

Figure 2. Site locations for the four littoral-zone microhabitats investigated at Sullivan Lake, Minn. Numbers in parentheses (indicate microhabitat groundwater/surface water sampling locations in meters parallel $(\mathrm{x})$ and perpendicular $(\mathrm{y})$ to the shoreline. 

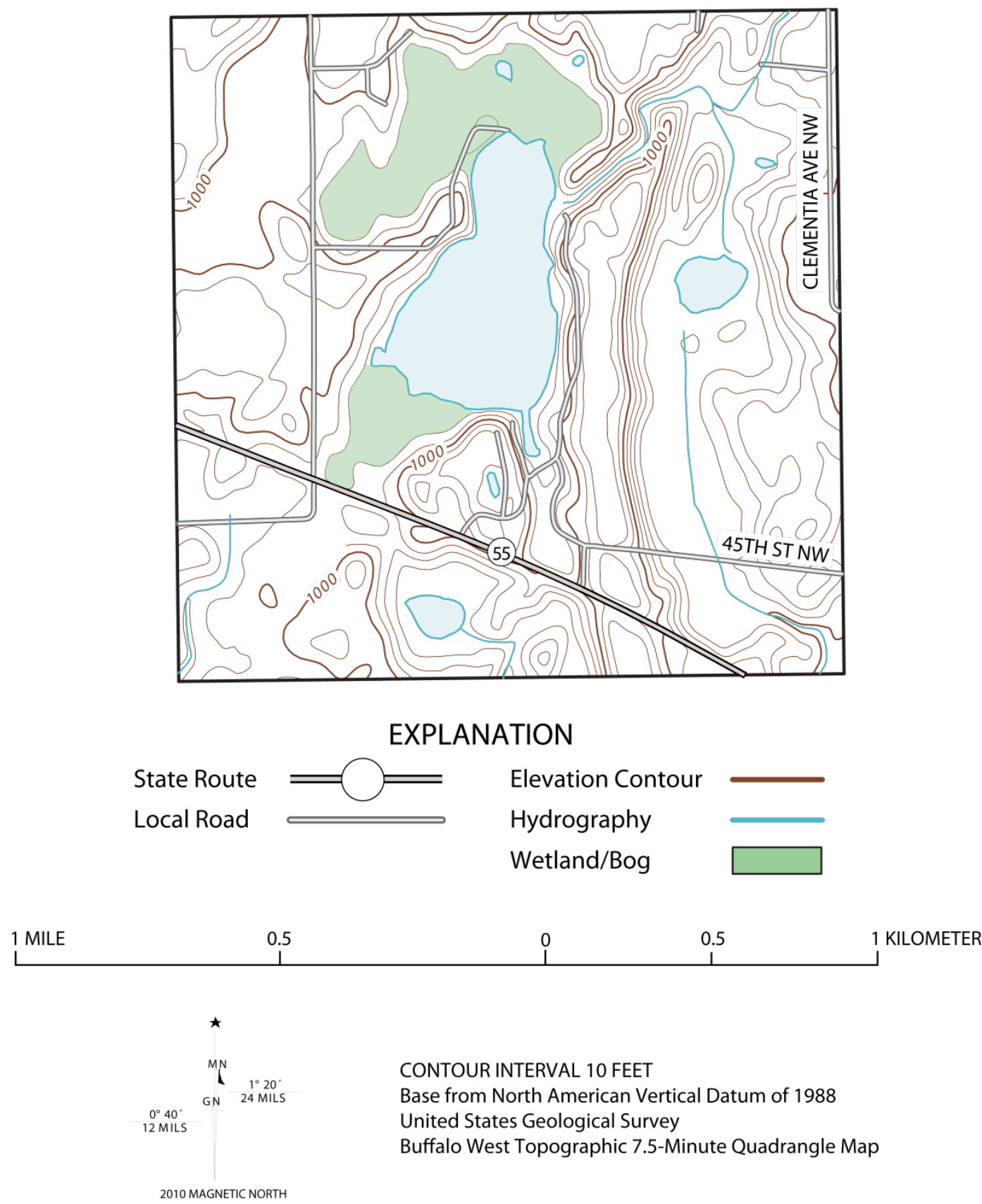

Figure 3. Site topography and preliminary hydraulic analysis of potential zones of groundwater discharge in Sullivan Lake, Minn. 
Table 1. Site characteristics for the 14 Minnesota lakes that were sampled in this investigation.

[DOW, Division of Wildlife; km2, square kilometer; m, meter; m3, cubic meter; $\mu \mathrm{g} / \mathrm{L}$, microgram per liter; km, kilometer; n/a, not applicable; Eu; eutrophic; Meso, mesotrophic; Oligo, oligotrophic; POCIS, polar organic compound integrated samplers; --, not available; Date format: M/DD/YY ]

\begin{tabular}{|c|c|c|c|c|c|c|c|c|c|c|c|c|c|c|c|}
\hline & Unit & Owasso & Cedar & Budd & Red Sand & Sullivan & $\begin{array}{l}\text { White } \\
\text { Sand }\end{array}$ & Kabetogama & Shingobee & Stewart & $\begin{array}{l}\text { Northern } \\
\text { Light }\end{array}$ & Elk & Beast & Ryan & Bohall \\
\hline Latitude & & 45.0313 & 44.9562 & 43.6397 & 46.3810 & 45.2200 & 46.3599 & 48.4442 & 46.9994 & 47.1825 & 47.9081 & 47.1965 & 48.5077 & 48.51881 & 47.2087 \\
\hline Longitude & & -93.1300 & -93.3254 & -94.4744 & -94.2861 & -93.9423 & -94.2874 & -93.0284 & -94.6874 & -91.7547 & -90.2521 & -95.2216 & -92.7538 & -92.7064 & -95.2484 \\
\hline $\begin{array}{l}\text { Lake DOW } \\
\text { number }\end{array}$ & & 62005600 & 27003900 & 46003000 & 18038600 & 86011900 & 18037900 & 69084500 & 29004300 & 38074400 & 16008900 & 15001000 & 69083700 & 69083500 & 15000900 \\
\hline County & & Ramsey & Hennepin & Martin & Crow Wing & Wright & Crow Wing & St. Louis & Hubbard & Lake & Cook & Clearwater & St. Louis & St. Louis & Clearwater \\
\hline $\begin{array}{l}\text { Hydrologic } \\
\text { class }\end{array}$ & & Headwater & Drainage & Drainage & Drainage & Headwater & Headwater & Riverine & Drainage & Drainage & Riverine & Drainage & $\mathrm{n} / \mathrm{a}$ & $\mathrm{n} / \mathrm{a}$ & $\mathrm{n} / \mathrm{a}$ \\
\hline $\begin{array}{l}\text { Watershed } \\
\text { area }\end{array}$ & $\mathrm{km}^{2}$ & 12 & 10 & 107 & 18 & 3 & 5 & 717 & 42 & 5 & 478 & 35 & $\mathrm{n} / \mathrm{a}$ & $\mathrm{n} / \mathrm{a}$ & $\mathrm{n} / \mathrm{a}$ \\
\hline $\begin{array}{l}\text { Lake surface } \\
\text { area }\end{array}$ & $\mathrm{km}^{2}$ & 1.64 & .72 & .94 & 2.17 & .31 & 1.81 & 104 & .75 & 1.03 & 1.97 & 1.33 & 0.33 & 0.14 & 0.15 \\
\hline $\begin{array}{l}\text { Maximum } \\
\text { depth }\end{array}$ & $\mathrm{m}$ & 11.3 & 15.5 & 5.18 & 7 & 16.5 & 7.9 & 23.8 & 11 & 6.1 & 2.3 & 28.4 & 20.1 & 3.7 & NA \\
\hline Lake volume & $\mathrm{m}^{3}$ & $5.0 \mathrm{E}+06$ & $4.0 \mathrm{E}+06$ & $3.1 \mathrm{E}+06$ & $2.3 \mathrm{E}+06$ & $1.3 \mathrm{E}+06$ & $5.0 \mathrm{E}+06$ & $7.3 \mathrm{E}+08$ & $4.0 \mathrm{E}+06$ & $1.9 \mathrm{E}+06$ & $2.3 \mathrm{E}+06$ & $1.3 \mathrm{E}+07$ & $\mathrm{n} / \mathrm{a}$ & $\mathrm{n} / \mathrm{a}$ & $\mathrm{n} / \mathrm{a}$ \\
\hline $\begin{array}{l}\text { Volume/ } \\
\text { watershed } \\
\text { area }\end{array}$ & & .410 & .395 & .029 & .125 & .420 & .983 & 1.023 & .096 & .388 & .005 & .364 & $\mathrm{n} / \mathrm{a}$ & $\mathrm{n} / \mathrm{a}$ & $\mathrm{n} / \mathrm{a}$ \\
\hline Shoreline & $\mathrm{m}$ & $9.1 \mathrm{E}+03$ & $4.9 \mathrm{E}+03$ & $3.6 \mathrm{E}+03$ & $6.1 \mathrm{E}+03$ & $2.7 \mathrm{E}+03$ & $5.4 \mathrm{E}+03$ & $1.8 \mathrm{E}+05$ & $4.9 \mathrm{E}+03$ & $5.1 \mathrm{E}+03$ & -- & -- & $\mathrm{n} / \mathrm{a}$ & $\mathrm{n} / \mathrm{a}$ & $\mathrm{n} / \mathrm{a}$ \\
\hline Trophic index & & $\mathrm{Eu}$ & $\mathrm{Eu}$ & $\mathrm{Eu}$ & $\mathrm{Eu}$ & $\mathrm{Eu}$ & Meso & Meso & Meso & Meso & Oligo & Oligo & Oligo & Oligo & Oligo \\
\hline $\begin{array}{l}\text { Total } \\
\text { phosphorous }\end{array}$ & $\mu \mathrm{g} / \mathrm{L}$ & 39 & 24 & 89 & 26 & 48 & 21 & 15 & 31 & 14 & 13 & 11 & 23 & 12 & NA \\
\hline $\begin{array}{l}\text { Septic } \\
\text { systems }\end{array}$ & number & 0 & 0 & 0 & 68 & 42 & 138 & 100 & 3 & 3 & 0 & 0 & 0 & 0 & 0 \\
\hline $\begin{array}{c}\text { Docks and } \\
\text { marinas }\end{array}$ & number & 164 & 5 & 80 & 32 & 6 & 85 & 242 & 1 & 13 & 0 & 1 & 0 & 0 & 0 \\
\hline $\begin{array}{l}\text { Shoreline } \\
\text { septic } \\
\text { density }\end{array}$ & $\mathrm{per} / \mathrm{km}$ & .0 & .0 & .0 & 11.2 & 15.4 & 25.6 & .6 & .6 & .6 & .0 & .0 & 0 & 0 & 0 \\
\hline $\begin{array}{l}\text { Shoreline } \\
\text { dock density }\end{array}$ & $\mathrm{per} / \mathrm{km}$ & 18.1 & 1.0 & 22.3 & 5.2 & 2.2 & 15.8 & 1.4 & .2 & 2.6 & .0 & .2 & 0 & 0 & 0 \\
\hline $\begin{array}{l}\text { Total length } \\
\text { of streams }\end{array}$ & $\mathrm{km}$ & $2,508.9$ & 112.2 & $48,797.0$ & $1,953.3$ & 15.3 & .0 & $391,614.1$ & $6,907.3$ & 24.2 & $227,398.2$ & $5,010.6$ & $\mathrm{n} / \mathrm{a}$ & $\mathrm{n} / \mathrm{a}$ & $\mathrm{n} / \mathrm{a}$ \\
\hline $\begin{array}{l}\text { Total length } \\
\text { of roads }\end{array}$ & $\mathrm{km}$ & $94,722.2$ & $95,536.9$ & $163,712.4$ & $54,841.7$ & $6,841.4$ & $23,179.0$ & $97,550.2$ & $48,231.9$ & 400.4 & $92,969.5$ & $6,283.6$ & $\mathrm{n} / \mathrm{a}$ & $\mathrm{n} / \mathrm{a}$ & $\mathrm{n} / \mathrm{a}$ \\
\hline $\begin{array}{l}\text { Total } \\
\text { population }\end{array}$ & persons & $13,227.0$ & $13,329.7$ & $5,805.3$ & $2,162.2$ & 168.9 & $1,345.3$ & 264.3 & 391.4 & 1.5 & 51.5 & 2.0 & $\mathrm{n} / \mathrm{a}$ & $\mathrm{n} / \mathrm{a}$ & $\mathrm{n} / \mathrm{a}$ \\
\hline $\begin{array}{l}\text { Population } \\
\text { density }\end{array}$ & person $/ \mathrm{km}^{2}$ & $1,078.2$ & $1,327.9$ & 54.3 & 117.4 & 55.1 & 264.6 & .4 & 9.3 & .3 & .1 & .1 & $\mathrm{n} / \mathrm{a}$ & $\mathrm{n} / \mathrm{a}$ & $\mathrm{n} / \mathrm{a}$ \\
\hline Road density & $\mathrm{km} / \mathrm{km}^{2}$ & 7.7 & 9.5 & 1.5 & 3.0 & 2.2 & 4.6 & .1 & 1.1 & .1 & .2 & .2 & 0 & 0 & $\mathrm{n} / \mathrm{a}$ \\
\hline $\begin{array}{l}\text { Stream } \\
\text { density }\end{array}$ & $\mathrm{km} / \mathrm{km}^{2}$ & .205 & .011 & .457 & .106 & .005 & .000 & .546 & .164 & .005 & .476 & .141 & $\mathrm{n} / \mathrm{a}$ & $\mathrm{n} / \mathrm{a}$ & $\mathrm{n} / \mathrm{a}$ \\
\hline $\begin{array}{l}\text { Developed } \\
\text { cover }\end{array}$ & percent & 73 & 80 & 10 & 10 & 8 & 19 & 0 & 4 & 0 & 0 & 1 & $\mathrm{n} / \mathrm{a}$ & $\mathrm{n} / \mathrm{a}$ & $\mathrm{n} / \mathrm{a}$ \\
\hline $\begin{array}{l}\text { Deciduous } \\
\text { cover }\end{array}$ & percent & 9 & 8 & 0 & 45 & 11 & 24 & 54 & 45 & 38 & 24 & 60 & $\mathrm{n} / \mathrm{a}$ & $\mathrm{n} / \mathrm{a}$ & $\mathrm{n} / \mathrm{a}$ \\
\hline $\begin{array}{l}\text { Evergreen/ } \\
\text { mixed cover }\end{array}$ & percent & 2 & 1 & $\mathrm{n} / \mathrm{a}$ & 1 & 1 & 0 & 16 & 21 & 38 & 57 & 26 & $\mathrm{n} / \mathrm{a}$ & $\mathrm{n} / \mathrm{a}$ & $\mathrm{n} / \mathrm{a}$ \\
\hline
\end{tabular}


Table 1. Site characteristics for the 14 Minnesota lakes that were sampled in this investigation._-Continued

[DOW, Division of Wildlife; km2, square kilometer; m, meter; m3, cubic meter; $\mu \mathrm{g} / \mathrm{L}$, microgram per liter; km, kilometer; n/a, not applicable; Eu; eutrophic; Meso, mesotrophic; Oligo, oligotrophic; POCIS, polar organic compound integrated samplers; --, not available; Date format: M/DD/YY ]

\begin{tabular}{|c|c|c|c|c|c|c|c|c|c|c|c|c|c|c|c|}
\hline & Unit & Owasso & Cedar & Budd & Red Sand & Sullivan & $\begin{array}{l}\text { White } \\
\text { Sand }\end{array}$ & Kabetogama & Shingobee & Stewart & $\begin{array}{l}\text { Northern } \\
\text { Light }\end{array}$ & Elk & Beast & Ryan & Bohall \\
\hline $\begin{array}{l}\text { Grassland } \\
\text { cover }\end{array}$ & percent & 0 & 0 & 4 & 4 & 11 & 2 & 2 & 18 & 0 & 0 & 0 & $\mathrm{n} / \mathrm{a}$ & $\mathrm{n} / \mathrm{a}$ & $\mathrm{n} / \mathrm{a}$ \\
\hline $\begin{array}{l}\text { Cropland } \\
\text { cover }\end{array}$ & percent & $\mathrm{n} / \mathrm{a}$ & $\mathrm{n} / \mathrm{a}$ & 76 & 2 & 49 & 2 & 0 & 2 & $\mathrm{n} / \mathrm{a}$ & $\mathrm{n} / \mathrm{a}$ & 0 & $\mathrm{n} / \mathrm{a}$ & $\mathrm{n} / \mathrm{a}$ & $\mathrm{n} / \mathrm{a}$ \\
\hline Wetland cover & percent & 3 & 2 & 2 & 14 & 7 & 18 & 10 & 2 & 1 & 4 & 1 & $\mathrm{n} / \mathrm{a}$ & $\mathrm{n} / \mathrm{a}$ & $\mathrm{n} / \mathrm{a}$ \\
\hline $\begin{array}{l}\text { Open water } \\
\text { cover }\end{array}$ & percent & 14 & 7 & 6 & 22 & 8 & 34 & 14 & 6 & 21 & 12 & 12 & $\mathrm{n} / \mathrm{a}$ & $\mathrm{n} / \mathrm{a}$ & $\mathrm{n} / \mathrm{a}$ \\
\hline Barren cover & percent & $\mathrm{n} / \mathrm{a}$ & $\mathrm{n} / \mathrm{a}$ & 0 & $\mathrm{n} / \mathrm{a}$ & $\mathrm{n} / \mathrm{a}$ & $\mathrm{n} / \mathrm{a}$ & 0 & $\mathrm{n} / \mathrm{a}$ & $\mathrm{n} / \mathrm{a}$ & 0 & $\mathrm{n} / \mathrm{a}$ & $\mathrm{n} / \mathrm{a}$ & $\mathrm{n} / \mathrm{a}$ & $\mathrm{n} / \mathrm{a}$ \\
\hline $\begin{array}{l}\text { Shrubland } \\
\text { cover }\end{array}$ & percent & $\mathrm{n} / \mathrm{a}$ & 0 & $\mathrm{n} / \mathrm{a}$ & 1 & 5 & $\mathrm{n} / \mathrm{a}$ & 3 & 2 & 1 & 3 & 0 & $\mathrm{n} / \mathrm{a}$ & $\mathrm{n} / \mathrm{a}$ & $\mathrm{n} / \mathrm{a}$ \\
\hline $\begin{array}{l}\text { POCIS } \\
\text { deployment }\end{array}$ & date & $5 / 23 / 08$ & $5 / 23 / 08$ & $5 / 27 / 08$ & $6 / 18 / 08$ & $6 / 19 / 08$ & $6 / 18 / 08$ & $8 / 21 / 08$ & $7 / 15 / 08$ & $7 / 14 / 08$ & $8 / 4 / 08$ & $6 / 18 / 08$ & $\mathrm{n} / \mathrm{a}$ & $\mathrm{n} / \mathrm{a}$ & $\mathrm{n} / \mathrm{a}$ \\
\hline $\begin{array}{l}\text { Water } \\
\text { sampling }\end{array}$ & date & $6 / 2 / 08$ & $6 / 2 / 08$ & $6 / 3 / 08$ & $6 / 27 / 08$ & $6 / 29 / 08$ & $6 / 27 / 08$ & $7 / 3 / 08$ & $7 / 15 / 08$ & $7 / 14 / 08$ & $8 / 4 / 08$ & $6 / 27 / 08$ & $10 / 19 / 10$ & $10 / 19 / 10$ & $10 / 21 / 10$ \\
\hline
\end{tabular}


Table 2. Trace elements and major elements measured by inductively coupled plasma/mass spectrometry (Garbarino and Taylor, 1996; Taylor, 2001) and inductively coupled plasma/atomic-emission spectrometry (Garbarino and Taylor, 1979) at the U.S. Geological Survey National Research Program Laboratory, and the trace and major elements' approximate method detection limits (MDL).

[atomic symbols shown in parenthesis; $\mu \mathrm{g} / \mathrm{L}$, microgram per liter; $\mathrm{mg} / \mathrm{L}$, milligram per liter]

\begin{tabular}{|c|c|c|c|}
\hline Element & MDL ( $\mu \mathrm{g} / \mathrm{L})$ & Element & MDL $(\mu \mathrm{g} / \mathrm{L})$ \\
\hline Aluminum (Al) & 0.05 & Manganese (Mn) & 0.02 \\
\hline Antimony (Sb) & .02 & Molybdenum (Mo) & .03 \\
\hline Arsenic (As) & .04 & Neodymium (Nd) & .003 \\
\hline Barium (Ba) & .01 & Nickel (Ni) & .02 \\
\hline Beryllium (Be) & .02 & Potassium (K) & $.02 \mathrm{mg} / \mathrm{L}$ \\
\hline Bismuth (Bi) & .01 & Praseodymium (Pr) & .005 \\
\hline Boron (B) & 4 & Rhenium (Re) & .001 \\
\hline Cadmium (Cd) & .006 & Rubidium (Rb) & .002 \\
\hline Calcium (Ca) & $.02 \mathrm{mg} / \mathrm{L}$ & Samarium (Sm) & .003 \\
\hline Cerium $(\mathrm{Ce})$ & .001 & Selenium (Se) & .2 \\
\hline Cesium (Cs) & .06 & Silica (SiO2) & $.1 \mathrm{mg} / \mathrm{L}$ \\
\hline Chromium (Cr) & .2 & Sodium $(\mathrm{Na})$ & $.02 \mathrm{mg} / \mathrm{L}$ \\
\hline Cobalt (Co) & .01 & Strontium (Sr) & .02 \\
\hline Copper $(\mathrm{Cu})$ & .02 & Sulfur (S) & $.002 \mathrm{mg} / \mathrm{L}$ \\
\hline Dysprosium (Dy) & .002 & Tellurium (Te) & .01 \\
\hline Erbium (Er) & .002 & Terbium (Tb) & .0003 \\
\hline Europium (Eu) & .001 & Thallium (Tl) & .005 \\
\hline Gadolinium (Gd) & .003 & Thorium (Th) & .004 \\
\hline Holmium (Ho) & .0005 & Thulium (Tm) & .0005 \\
\hline Iron $(\mathrm{Fe})$ & .7 & Tungsten (W) & .004 \\
\hline Lanthanum (La) & .0005 & Uranium (U) & .002 \\
\hline Lead $(\mathrm{Pb})$ & .006 & Vanadium (V) & .05 \\
\hline Lithium (Li) & .1 & Ytterbium (Yb) & .0014 \\
\hline Lutetium (Lu) & .0005 & Yttrium (Y) & .0004 \\
\hline \multirow[t]{2}{*}{ Magnesium (Mg) } & $.002 \mathrm{mg} / \mathrm{L}$ & Zinc (Zn) & .08 \\
\hline & & Zirconium (Zr) & .01 \\
\hline
\end{tabular}


Table 3. Acidic organic compounds measured by evaporation with derivatization and gas chromatography/mass spectrometry (Barber and others, 2000) at the U.S. Geological Survey National Research Program Laboratory.

[This report contains CAS Registry Numbers ${ }^{\circledR}$ (CASRN), which is a Registered Trademark of the American Chemical Society. CAS recommends the verification of the CASRNs through CAS Client ServicesSM; MDL, method detection limit; $\mu \mathrm{g} / \mathrm{L}$, microgram per liter; NA, not available; *, potential endocrine-disrupting compound]

\begin{tabular}{lcc}
\hline \multicolumn{1}{c}{ Compound } & CASRN & MDL $(\mathbf{\mu g} / \mathbf{L})$ \\
\hline Ethylenediaminetetraacetic acid & $60-00-4$ & 0.05 \\
Nitrilotriacetic acid & $139-13-9$ & .05 \\
4-Nonylphenolmonoethoxycarboxylic acid* & $\mathrm{NA}$ & .1 \\
4-Nonylphenoldiethoxycarboxylic acid* & $106807-78-7$ & .1 \\
4-Nonylphenoltriethoxycarboxylic acid* & $\mathrm{NA}$ & .1 \\
4-Nonylphenoltetraethoxycarboxylic acid* & $\mathrm{NA}$ & .1 \\
Surrogate Standard & & \\
4-normal-Nonylphenoldiethoxycarboxylate & $\mathrm{NA}$ & $\mathrm{NA}$ \\
\hline
\end{tabular}


Table 4. Neutral organic compounds measured by continuous liquid-liquid extraction using methylene chloride with gas chromatography/mass spectrometry (Barber and others, 2000) at the U.S. Geological Survey National Research Program Laboratory.

[This report contains CAS Registry Numbers ${ }^{\circledR}$ (CASRN), which is a Registered Trademark of the American Chemical Society. CAS recommends the verification of the CASRNs through CAS Client ServicesSM; MDL, method detection limit; $\mu \mathrm{g} / \mathrm{L}$, microgram per liter; NA, not available; *, potential endocrine-disrupting compound]

\begin{tabular}{lcc}
\hline \multicolumn{1}{c}{ Compound } & CASRN & MDL ( $\boldsymbol{\mu g} / \mathbf{L}$ ) \\
\hline Bisphenol A* & $80-05-7$ & 0.01 \\
2[3]-tert-Butyl-4-methylphenol & $25013-16-5$ & .01 \\
4-tert-Butylphenol & $98-54-4$ & .01 \\
Caffeine & $58-08-2$ & .01 \\
2,6-Di-tert-butyl-1,4-benzoquinone & $719-22-2$ & .01 \\
2,6-Di-tert-butyl-4-methylphenol & $128-37-0$ & .01 \\
2,6-Di-tert-butylphenol & $128-39-2$ & .01 \\
1,2-Dichlorobenzene & $95-50-1$ & .01 \\
1,3-Dichlorobenzene & $541-73-1$ & .01 \\
1,4-Dichlorobenzene* & $106-46-7$ & .01 \\
N,N-Diethyl-meta-toluamide & $134-62-3$ & .01 \\
4-Ethylphenol & $123-07-9$ & .01 \\
5-Methyl-1H-benzotriazole & $136-85-6$ & .01 \\
4-Methylphenol & $106-44-5$ & .01 \\
4-Nonylphenol * & $25154-52-3$ & .05 \\
4-Nonylphenolmonoethoxylate* & $9016-45-9$ & .05 \\
4-Nonylphenoldiethoxylate* & $\mathrm{NA}$ & .05 \\
4-Nonylphenoltriethoxylate* & $\mathrm{NA}$ & .05 \\
4-Nonylphenoltetraethoxylate* & $\mathrm{NA}$ & .05 \\
4-normal-Octylphenol* & $1806-26-4$ & .01 \\
4-tert-Octylphenol* & $140-66-9$ & .01 \\
4-tert-Octylphenolmonoethoxylate* & $9036-19-5$ & $\mathrm{NA}$ \\
4-tert-Octylphenoldiethoxylate* & $\mathrm{NA}$ & $\mathrm{NA}$ \\
4-tert-Octylphenoltriethoxylate* & $\mathrm{NA}$ & .01 \\
4-tert-Octylphenoltetraethoxylate* & $\mathrm{NA}$ & .01 \\
4-tert-Octylphenolpentaethoxylate* & $\mathrm{NA}$ & .01 \\
4-tert-Pentylphenol* & $80-46-6$ & .01 \\
4-Propylphenol & $645-56-7$ & .01 \\
Triclosan & $3380-34-5$ & .01 \\
Surrogate Standards & & .01 \\
d6 Bisphenol A & $86588-58-1$ & .01 \\
d21 2,6-Di-tert-butyl-4-methylphenol & $64502-99-4$ & $\mathrm{NA}$ \\
4-normal-Nonylphenol & $104-40-5$ & $\mathrm{NA}$ \\
4-normal-Nonylphenolmonoethoxylate & & \\
4-normal-Nonylphenoldiethoxylate & NA & \\
\hline
\end{tabular}


Figure 5. Steroid and steroidal hormone compounds measured by octadecylsilica solid-phase extraction with derivatization and gas chromatography/tandem mass spectrometry (Barber and others, 2003; Foreman and others, 2012) at the U.S. Geological Survey National Research Program Laboratory.

[This report contains CAS Registry Numbers ${ }^{\circledR}(\mathrm{CASRN})$, which is a Registered Trademark of the American Chemical Society. CAS recommends the verification of the CASRNs through CAS Client ServicesSM; MDL, method detection limit; $\mu \mathrm{g} / \mathrm{L}$, microgram per liter; *, potential endocrine-disrupting compound; NA, not available; --, not applicable]

\begin{tabular}{|c|c|c|}
\hline Compound & CASRN & MDL ( $\mu \mathrm{g} / \mathrm{L})$ \\
\hline 4-Androstene-3,17-dione & $63-05-8$ & 0.0001 \\
\hline cis-Androsterone & $53-41-8$ & .0001 \\
\hline Cholesterol & $57-88-5$ & .005 \\
\hline Coprostanol & $360-68-9$ & .005 \\
\hline Diethylstilbesterol* & $56-53-1$ & .0001 \\
\hline Equilenin* & 517-09-9 & .0001 \\
\hline Equilin* & $474-86-2$ & .0001 \\
\hline 17 $\alpha$-Estradiol* & $57-91-0$ & .0001 \\
\hline 17ß-Estradiol* & $50-28-2$ & .0001 \\
\hline Estriol* & $50-27-1$ & .0001 \\
\hline Estrone* & $53-16-7$ & .0001 \\
\hline 17 $\alpha$-Ethinylestradiol* & $57-63-6$ & .0001 \\
\hline Mestranol* & $72-33-3$ & .0001 \\
\hline 19-Norethisterone* & $68-22-4$ & .0001 \\
\hline Progesterone & $57-83-0$ & .0001 \\
\hline Testosterone & $58-22-0$ & .0001 \\
\hline dihydro-Testosterone & $521-18-6$ & .0001 \\
\hline epi-Testosterone & 481-30-1 & .0001 \\
\hline 11-keto-Testosterone & $53187-98-7$ & .0001 \\
\hline \multicolumn{3}{|l|}{ Surrogate Standards } \\
\hline d7-Androstenedione & $67034-85-9$ & -- \\
\hline d7-Cholesterol & $83199-47-7$ & -- \\
\hline d12-Chrysene & $1719-03-5$ & -- \\
\hline d4-Dihydrotestosterone & NA & -- \\
\hline d4-17 $\alpha$-Ethinylestradiol & NA & -- \\
\hline $\mathrm{d} 4-17 \beta$-Estradiol & $66789-03-5$ & -- \\
\hline d3-Estriol & $79037-36-8$ & -- \\
\hline d4-Estrone & $53866-34-5$ & -- \\
\hline d4-Mestranol & NA & -- \\
\hline d6-Norethindrone & NA & -- \\
\hline d9-Progesterone & $15775-74-3$ & -- \\
\hline d5-Testosterone & $21002-80-2$ & -- \\
\hline
\end{tabular}


Table 6. Summary of field measurements made at 11 Minnesota lakes during 2008.

[Date format: M/DD. $\mu \mathrm{S} / \mathrm{cm}$, microsiemen per centimeter, mg/L, milligram per liter; mg/L, std Unit, standard unit, mV, millivolts; NM, not measured]

\begin{tabular}{lccccccccc}
\hline \multicolumn{1}{c}{ Waterbody } & Date & $\begin{array}{c}\text { Water temp } \\
{ }^{\circ} \mathbf{C}\end{array}$ & $\begin{array}{c}\text { Specific } \\
\text { conductivity } \\
\mathbf{\mu S / c m}\end{array}$ & $\begin{array}{c}\text { Total } \\
\text { dissolved } \\
\text { solids } \\
\mathbf{m g} / \mathbf{L}\end{array}$ & $\begin{array}{c}\text { Salinity } \\
\text { per } \\
\text { thousands }\end{array}$ & $\begin{array}{c}\text { Dissolved } \\
\text { oxygen } \\
\text { percent }\end{array}$ & $\begin{array}{c}\text { Dissolved } \\
\text { oxygen } \\
\text { mg/L }\end{array}$ & $\begin{array}{c}\text { pH } \\
\text { std unit }\end{array}$ & $\begin{array}{c}\text { Oxidation } \\
\text { reduction } \\
\text { potential } \\
\text { mV }\end{array}$ \\
\hline Cedar & $5 / 23$ & 18.1 & 580 & 430 & 0.33 & 120 & 11 & 8.2 & 140 \\
Cedar & $6 / 2$ & 19.9 & 550 & 400 & .3 & 140 & 13 & 8.5 & 150 \\
Cedar & $6 / 13$ & 20.1 & 490 & 350 & .26 & 100 & 9.5 & 7.5 & 140 \\
Cedar & $7 / 10$ & 25.4 & 540 & 350 & .26 & 83 & 6.6 & 8.8 & 130 \\
Owasso Lake & $5 / 23$ & 16.6 & 330 & 260 & .19 & NM & 10 & 7.6 & 150 \\
Owasso Lake & $6 / 2$ & 20.0 & 330 & 280 & .18 & 140 & 12 & 8.2 & 150 \\
Owasso Lake & $6 / 13$ & 19.9 & 300 & 220 & .16 & 89 & 8.1 & 7.3 & 140 \\
Budd Lake & $5 / 22$ & 16.2 & 430 & 340 & .25 & 96 & 9.4 & 10 & 120 \\
Budd Lake & $6 / 3$ & 18.8 & 490 & 360 & .27 & NM & 9.0 & 8.3 & 160 \\
Budd Lake & $6 / 12$ & 20.5 & 420 & 300 & .22 & 94 & 8.4 & 9.3 & 140 \\
White Sand & $7 / 9$ & 26.2 & 140 & 86 & .06 & 95 & 7.6 & 7.9 & 110 \\
Red Sand Lake & $6 / 18$ & 24.1 & 53 & 37 & .03 & 120 & 10 & 2.5 & 240 \\
Red Sand Lake & $7 / 9$ & 26.9 & 170 & 100 & .07 & 100 & 8.3 & 8.1 & 120 \\
Sullivan Lake & $6 / 18$ & 21.8 & 89 & 60 & .05 & 92 & 7.9 & 2.2 & 260 \\
Elk Lake & $6 / 18$ & 18.2 & 220 & 160 & .12 & 120 & 11 & 7.8 & 120 \\
Elk Lake & $7 / 9$ & 21.8 & 250 & 180 & .13 & 93 & 8.1 & 7.7 & 130 \\
Stewart Lake & $7 / 14$ & 20.3 & 48 & 35 & .02 & 96 & 8.5 & 7.8 & 68 \\
Shingobee Lake & $7 / 14$ & 21.1 & 280 & 200 & .14 & 85 & 7.6 & 7.6 & 140 \\
Lake Kabetogama & $7 / 2$ & 18.6 & 67 & 50 & .03 & 97 & 9.1 & 1.2 & 280 \\
Northern Light Lake & $8 / 3$ & 21.8 & 33 & 25 & .02 & 91 & 8.0 & NM & 110 \\
\hline
\end{tabular}


Table 7. Summary of major- and trace-element data for water samples collected from 11 Minnesota lakes during 2008.

$[\mathrm{mg} / \mathrm{L}$, milligram per liter; $\mu \mathrm{g} / \mathrm{L}$, microgram per liter; $<$, less than]

\begin{tabular}{|c|c|c|c|c|c|c|c|c|c|c|c|c|}
\hline Constituent & Unit & Owasso & Cedar & Budd & Sullivan & Red Sand & Shingobee & $\begin{array}{l}\text { White } \\
\text { Sand }\end{array}$ & Stewart & Kabetogama & $\begin{array}{c}\text { Northern } \\
\text { Light }\end{array}$ & Elk \\
\hline Aluminum & $\mu \mathrm{g} / \mathrm{L}$ & 16 & 7.7 & 8.2 & 2.3 & 3.3 & 0.3 & 1.0 & 7.0 & 10 & 28 & 1.7 \\
\hline Antimony & $\mu \mathrm{g} / \mathrm{L}$ & .39 & .23 & .22 & .074 & .033 & .015 & .042 & .039 & .048 & .032 & .021 \\
\hline Arsenic & $\mu \mathrm{g} / \mathrm{L}$ & 11 & 1.9 & 1.2 & 1.3 & .93 & .71 & .63 & .45 & .38 & .39 & .91 \\
\hline Barium & $\mu \mathrm{g} / \mathrm{L}$ & 38 & 38 & 55 & 34 & 28 & 59 & 36 & 10 & 12 & 4.8 & 45 \\
\hline Beryllium & $\mu \mathrm{g} / \mathrm{L}$ & .008 & .006 & $<.005$ & $<.005$ & .006 & $<.005$ & $<.005$ & .005 & .008 & .016 & $<.005$ \\
\hline Bismuth & $\mu \mathrm{g} / \mathrm{L}$ & $<.006$ & $<.006$ & $<.006$ & $<.006$ & $<.006$ & $<.006$ & $<.006$ & $<.006$ & $<.006$ & $<.006$ & $<.006$ \\
\hline Boron & $\mu \mathrm{g} / \mathrm{L}$ & 22 & 20 & 33 & 26 & 16 & 26 & 16 & 14 & 7 & 8 & 27 \\
\hline Cadmium & $\mu \mathrm{g} / \mathrm{L}$ & .055 & .038 & .015 & $<.002$ & .007 & .009 & .004 & .011 & .010 & .015 & .020 \\
\hline Cesium & $\mu \mathrm{g} / \mathrm{L}$ & .05 & .04 & .03 & .03 & .03 & .02 & .04 & .01 & .04 & .02 & .03 \\
\hline Calcium & $\mathrm{mg} / \mathrm{L}$ & 25 & 36 & 57 & 38 & 15 & 46 & 25 & 14 & 11 & 4.5 & 34 \\
\hline Cerium & $\mu \mathrm{g} / \mathrm{L}$ & .021 & .011 & .015 & .0036 & .0091 & .0039 & .0098 & .018 & .055 & .18 & .0038 \\
\hline Chromium & $\mu \mathrm{g} / \mathrm{L}$ & $<1$ & $<1$ & $<1$ & $<1$ & $<1$ & $<1$ & $<1$ & $<1$ & $<1$ & $<1$ & $<1$ \\
\hline Cobalt & $\mu \mathrm{g} / \mathrm{L}$ & .036 & .051 & .050 & .032 & .029 & .0031 & .016 & .014 & .048 & .031 & .016 \\
\hline Copper & $\mu \mathrm{g} / \mathrm{L}$ & 2.4 & .81 & 2.5 & .25 & .37 & .50 & 1.5 & .93 & .90 & 2.5 & .19 \\
\hline Dysprosium & $\mu \mathrm{g} / \mathrm{L}$ & .0024 & .0017 & .0025 & .0007 & .0014 & .0003 & .0008 & .0037 & .0057 & .028 & .0003 \\
\hline Erbium & $\mu \mathrm{g} / \mathrm{L}$ & .0016 & .0006 & .0021 & .0003 & .0007 & $<.0001$ & .0007 & .0019 & .0037 & .017 & .0001 \\
\hline Europium & $\mu \mathrm{g} / \mathrm{L}$ & .0013 & .0009 & $<.0001$ & .0008 & .0008 & .0004 & .0011 & .0007 & .0022 & .0080 & .0017 \\
\hline Gadolinium & $\mu \mathrm{g} / \mathrm{L}$ & .0024 & .0011 & .0029 & .0012 & .0009 & .0006 & .0012 & .0034 & .0084 & .039 & .0005 \\
\hline Holmium & $\mu \mathrm{g} / \mathrm{L}$ & .0004 & .0003 & .0004 & .0002 & .0003 & .0001 & .0002 & .0006 & .0014 & .0059 & .0001 \\
\hline Iron & $\mu \mathrm{g} / \mathrm{L}$ & 30 & 7 & 11 & 9 & 204 & $<2$ & 112 & 33 & 23 & 376 & 137 \\
\hline Lanthanum & $\mu \mathrm{g} / \mathrm{L}$ & .018 & .0083 & .0097 & .0037 & .0071 & .0030 & .0069 & .014 & .056 & .20 & .0026 \\
\hline Lead & $\mu \mathrm{g} / \mathrm{L}$ & .60 & .33 & .20 & .17 & .12 & .05 & .07 & .06 & .12 & .16 & 90 \\
\hline Lithium & $\mu \mathrm{g} / \mathrm{L}$ & 2.3 & 12 & 18 & 13 & 1.1 & 4.0 & .85 & 1.0 & 1.1 & .77 & 4.2 \\
\hline Lutetium & $\mu \mathrm{g} / \mathrm{L}$ & .0002 & .0002 & .0004 & .0001 & .0004 & .0000 & .0003 & .0003 & .0004 & .0026 & .0001 \\
\hline Magnesium & $\mathrm{mg} / \mathrm{L}$ & 6.0 & 9.6 & 28 & 19 & 5.4 & 16 & 6.1 & 4.5 & 3.9 & 1.8 & 16 \\
\hline Manganese & $\mu \mathrm{g} / \mathrm{L}$ & 2.8 & 5.3 & 7.0 & 1.1 & 4.8 & .26 & 1.0 & 1.1 & 2.1 & 5.4 & 3.3 \\
\hline Molybdenum & $\mu \mathrm{g} / \mathrm{L}$ & 0.63 & 0.91 & 2.6 & 0.43 & 0.04 & 0.38 & 0.08 & 0.20 & 0.18 & 0.17 & 0.36 \\
\hline Neodymium & $\mu \mathrm{g} / \mathrm{L}$ & .018 & .0069 & .0097 & .0020 & .0051 & .0021 & .0050 & .014 & .045 & .25 & .0027 \\
\hline Nickel & $\mu \mathrm{g} / \mathrm{L}$ & 1.1 & 1.0 & 1.5 & 1.1 & .37 & .90 & .60 & .48 & .70 & .63 & .67 \\
\hline Potassium & $\mathrm{mg} / \mathrm{L}$ & 3.6 & 2.9 & 2.3 & 4.0 & .51 & 1.5 & .40 & .52 & .89 & .25 & 1.5 \\
\hline Praseodymium & $\mu \mathrm{g} / \mathrm{L}$ & .0039 & .0027 & .0021 & .0005 & .0012 & .0008 & .0014 & .0036 & .011 & .059 & .0004 \\
\hline
\end{tabular}


Table 7. Summary of major- and trace-element data for water samples collected from 11 Minnesota lakes during 2008.—Continued $[\mathrm{mg} / \mathrm{L}$, milligram per liter; $\mu \mathrm{g} / \mathrm{L}$, microgram per liter; <, less than $]$

\begin{tabular}{|c|c|c|c|c|c|c|c|c|c|c|c|c|}
\hline Constituent & Unit & Owasso & Cedar & Budd & Sullivan & Red Sand & Shingobee & $\begin{array}{l}\text { White } \\
\text { Sand }\end{array}$ & Stewart & Kabetogama & $\begin{array}{c}\text { Northern } \\
\text { Light }\end{array}$ & Elk \\
\hline Beryllium & $\mu \mathrm{g} / \mathrm{L}$ & .008 & .006 & $<.005$ & $<.005$ & .006 & $<.005$ & $<.005$ & .005 & .008 & .016 & $<.005$ \\
\hline Rhenium & $\mu \mathrm{g} / \mathrm{L}$ & .002 & .003 & .040 & .012 & .001 & .001 & .001 & .002 & .001 & .001 & $<.001$ \\
\hline Rubidium & $\mu \mathrm{g} / \mathrm{L}$ & 2.0 & 2.2 & .89 & 2.4 & .98 & .98 & .53 & .55 & 1.2 & .53 & 1.0 \\
\hline Samarium & $\mu \mathrm{g} / \mathrm{L}$ & .0039 & .0021 & .0025 & .0005 & .0014 & .0006 & .0012 & .0033 & .0085 & .046 & .0005 \\
\hline Selenium & $\mu \mathrm{g} / \mathrm{L}$ & .4 & .2 & 1.4 & .2 & .2 & $<.1$ & .1 & .1 & $<.1$ & .1 & .3 \\
\hline Silica dioxide & $\mathrm{mg} / \mathrm{L}$ & .10 & .22 & 6.2 & 4.4 & 2.9 & 13 & 1.8 & 3.9 & 4.1 & 4.9 & 9.3 \\
\hline Sodium & $\mathrm{mg} / \mathrm{L}$ & 30 & 64 & 6.5 & 7.9 & 8.0 & 4.9 & 7.5 & 1.8 & 1.6 & 1.0 & 6.0 \\
\hline Strontium & $\mu \mathrm{g} / \mathrm{L}$ & 42 & 59 & 202 & 106 & 37 & 82 & 46 & 35 & 24 & 18 & 74 \\
\hline Sulfur & $\mathrm{mg} / \mathrm{L}$ & 1.7 & 4.3 & 14 & 2.5 & .46 & 1.6 & .48 & 1.1 & 1.4 & 1.1 & .19 \\
\hline Tellerium & $\mu \mathrm{g} / \mathrm{L}$ & $<.007$ & $<.007$ & .009 & $<.007$ & .007 & $<.007$ & $<.007$ & $<.007$ & $<.007$ & $<.007$ & $<.007$ \\
\hline Terbium & $\mu \mathrm{g} / \mathrm{L}$ & .0005 & .0003 & .0003 & .0001 & .0002 & .0001 & .0001 & .0004 & .0011 & .0050 & .0000 \\
\hline Thallium & $\mu \mathrm{g} / \mathrm{L}$ & .0044 & .0038 & .0066 & .0031 & .0031 & .0010 & .0040 & .0023 & .012 & .0076 & .0027 \\
\hline Thorium & $\mu \mathrm{g} / \mathrm{L}$ & $<.02$ & $<.02$ & $<.02$ & $<.02$ & $<.02$ & $<.02$ & $<.02$ & $<.02$ & $<.02$ & $<.02$ & $<.02$ \\
\hline Thulium & $\mu \mathrm{g} / \mathrm{L}$ & .0002 & .0001 & .0003 & .0001 & .0001 & .0001 & .0001 & .0005 & .0004 & .0022 & .0001 \\
\hline Tin & $\mu \mathrm{g} / \mathrm{L}$ & .046 & .013 & .009 & $<.008$ & $<.008$ & $<.008$ & $<.008$ & $<.008$ & $<.008$ & .025 & .015 \\
\hline Tungsten & $\mu \mathrm{g} / \mathrm{L}$ & .0061 & .047 & .012 & .0023 & .0047 & .0021 & $<.001$ & .0018 & .0093 & $<.001$ & $<.001$ \\
\hline Uranium & $\mu \mathrm{g} / \mathrm{L}$ & .091 & .34 & 8.9 & .56 & .011 & .13 & .018 & .037 & .060 & .019 & .021 \\
\hline Vanadium & $\mu \mathrm{g} / \mathrm{L}$ & .47 & .4 & 1.6 & .16 & .13 & $<.1$ & $<.1$ & .19 & .30 & .40 & $<.1$ \\
\hline Ytterbium & $\mu \mathrm{g} / \mathrm{L}$ & .0011 & .0009 & .0018 & .0006 & .0008 & $<.0003$ & .0011 & .0018 & .0043 & .016 & $<.0003$ \\
\hline Yttrium & $\mu \mathrm{g} / \mathrm{L}$ & .015 & .0091 & .020 & .0048 & .0072 & .0031 & .0066 & .026 & .041 & .18 & .0035 \\
\hline Zinc & $\mu \mathrm{g} / \mathrm{L}$ & 5.9 & 11 & 3.4 & 1.7 & 1.7 & .92 & 1.3 & 7.9 & 11 & 28 & 14 \\
\hline Zirconium & $\mu \mathrm{g} / \mathrm{L}$ & .05 & .03 & .13 & .04 & .02 & .06 & .04 & .03 & .06 & .09 & .03 \\
\hline
\end{tabular}


Table 8. Summary of acidic organic compound data for water samples collected from 11 Minnesota lakes during 2008.

$[\mu \mathrm{g} / \mathrm{L}$, microgram per liter; $<$, less than; Dup, duplicate sample $]$

\begin{tabular}{|c|c|c|c|c|c|c|c|c|c|c|c|c|}
\hline Compound & $\begin{array}{c}\text { Owasso } \\
\mu \mathrm{g} / \mathrm{L}\end{array}$ & $\begin{array}{c}\text { Cedar } \\
\mu \mathrm{g} / \mathrm{L}\end{array}$ & $\begin{array}{l}\text { Budd } \\
\mu g / L\end{array}$ & $\begin{array}{l}\text { Budd } \\
\text { Dup } \\
\mu g / L\end{array}$ & $\begin{array}{c}\text { Sullivan } \\
\mu \mathrm{g} / \mathrm{L}\end{array}$ & $\begin{array}{l}\text { Red } \\
\text { Sand } \\
\mu g / L\end{array}$ & $\begin{array}{c}\text { Shingobee } \\
\mu \mathrm{g} / \mathrm{L}\end{array}$ & $\begin{array}{l}\text { White } \\
\text { Sand } \\
\mu \mathrm{g} / \mathrm{L}\end{array}$ & $\begin{array}{c}\text { Stewart } \\
\mu \mathrm{g} / \mathrm{L}\end{array}$ & $\begin{array}{c}\text { Kabetogama } \\
\mu \mathrm{g} / \mathrm{L}\end{array}$ & $\begin{array}{c}\text { Northern } \\
\text { Light } \\
\mu \mathrm{g} / \mathrm{L}\end{array}$ & $\begin{array}{l}\text { Elk } \\
\mu \mathrm{g} / \mathrm{L}\end{array}$ \\
\hline Ethylenediaminetetraacetic acid & 0.35 & 0.19 & 0.12 & 0.14 & 0.39 & 0.21 & 0.11 & 0.11 & 0.05 & 0.06 & 0.24 & 0.13 \\
\hline Nitrilotriacetic acid & $<.05$ & .07 & $<.05$ & $<.05$ & $<.05$ & $<.05$ & $<.05$ & $<.05$ & $<.05$ & $<.05$ & $<.05$ & $<.05$ \\
\hline 4-Nonylphenolmonoethoxycarboxylic acid & $<.1$ & .27 & $<.1$ & $<.1$ & $<.1$ & $<.1$ & .18 & $<.1$ & $<.1$ & $<.1$ & $<.1$ & $<.1$ \\
\hline 4-Nonylphenoldiethoxycarboxylic acid & $<.1$ & .14 & $<.1$ & $<.1$ & $<.1$ & $<.1$ & $<.1$ & $<.1$ & $<.1$ & $<.1$ & $<.1$ & $<.1$ \\
\hline 4-Nonylphenoltriethoxycarboxylic acid & $<.1$ & $<.1$ & $<.1$ & $<.1$ & $<.1$ & $<.1$ & $<.1$ & $<.1$ & $<.1$ & $<.1$ & $<.1$ & $<.1$ \\
\hline 4-Nonylphenoltetraethoxycarboxylic acid & $<.1$ & $<.1$ & $<.1$ & $<.1$ & $<.1$ & $<.1$ & $<.1$ & $<.1$ & $<.1$ & $<.1$ & $<.1$ & $<.1$ \\
\hline \multicolumn{13}{|l|}{ Surrogate Recovery (percent) } \\
\hline 4-normal-Nonylphenoldiethoxycarboxylic acid & 44 & 49 & 36 & 30 & 32 & 39 & 39 & 38 & 40 & 38 & 32 & 43 \\
\hline
\end{tabular}

Table 9. Summary of neutral organic compound data for water samples collected from 11 Minnesota lakes during 2008.

[avg, average; $\mu \mathrm{g} / \mathrm{L}$, microgram per liter; $<$, less than; Dup, duplicate sample; MS, matrix spike recovery in percent]

\begin{tabular}{|c|c|c|c|c|c|c|c|c|c|c|c|}
\hline Compound & $\begin{array}{c}\text { Owasso } \\
\mu \mathrm{g} / \mathrm{L}\end{array}$ & $\begin{array}{c}\text { Cedar } \\
\mu \mathrm{g} / \mathrm{L}\end{array}$ & $\begin{array}{l}\text { Budd } \\
\mu g / L\end{array}$ & $\begin{array}{c}\text { Sullivan } \\
\mu \mathrm{g} / \mathrm{L}\end{array}$ & $\begin{array}{c}\text { Red Sand } \\
\text { (avg) } \\
\mu g / L\end{array}$ & $\begin{array}{c}\text { Shingobee } \\
\mu \mathrm{g} / \mathrm{L}\end{array}$ & $\begin{array}{c}\text { White Sand } \\
\mu \mathrm{g} / \mathrm{L}\end{array}$ & $\begin{array}{c}\text { Stewart } \\
\mu \mathrm{g} / \mathrm{L}\end{array}$ & $\begin{array}{c}\text { Kabetogama } \\
\text { (avg) } \\
\mu \mathrm{g} / \mathrm{L}\end{array}$ & $\begin{array}{l}\text { Northern } \\
\text { Light } \\
\text { (avg) } \\
\mu \mathrm{g} / \mathrm{L}\end{array}$ & $\begin{array}{c}\text { Elk } \\
\text { (avg) } \\
\mu g / L\end{array}$ \\
\hline Bisphenol A & 0.03 & $<0.01$ & 0.04 & $<0.01$ & $<0.01$ & 0.02 & $<0.01$ & 0.02 & $<0.01$ & 0.01 & $<0.01$ \\
\hline 2[3]-tert-Butyl-4-methylphenol & $<.01$ & $<.01$ & $<.01$ & $<.01$ & $<.01$ & $<.01$ & $<.01$ & $<.01$ & $<.01$ & $<.01$ & $<.01$ \\
\hline 4-tert-Butylphenol & $<.01$ & $<.01$ & $<.01$ & $<.01$ & $<.01$ & $<.01$ & $<.01$ & $<.01$ & $<.01$ & $<.01$ & $<.01$ \\
\hline Caffeine & .13 & .09 & .02 & $<.01$ & $<.01$ & .01 & $<.01$ & .01 & $<.01$ & .02 & .01 \\
\hline 1,2-Dichlorobenzene & $<.01$ & $<.01$ & $<.01$ & $<.01$ & $<.01$ & $<.01$ & $<.01$ & $<.01$ & $<.01$ & $<.01$ & $<.01$ \\
\hline 1,3-Dichlorobenzene & $<.01$ & $<.01$ & $<.01$ & $<.01$ & $<.01$ & $<.01$ & $<.01$ & $<.01$ & $<.01$ & $<.01$ & $<.01$ \\
\hline 1,4-Dichlorobenzene & $<.01$ & $<.01$ & $<.01$ & $<.01$ & $<.01$ & $<.01$ & $<.01$ & $<.01$ & $<.01$ & $<.01$ & $<.01$ \\
\hline 2,6-Di-tert-butyl-1,4-benzoquinone & $<.01$ & .14 & $<.01$ & .08 & .08 & $<.01$ & .09 & $<.01$ & $<.01$ & $<.01$ & .15 \\
\hline 2,6-Di-tert-butyl-4-methylphenol & .07 & .07 & .07 & $<.01$ & .02 & $<.01$ & .02 & $<.01$ & $<.01$ & .02 & .02 \\
\hline 2,6-Di-tert-butylphenol & $<.01$ & $<.01$ & .18 & $<.01$ & $<.01$ & $<.01$ & $<.01$ & $<.01$ & $<.01$ & $<.01$ & $<.01$ \\
\hline$N, N$-Diethyl-meta-toluamide & .09 & .05 & .03 & .03 & .01 & .58 & .03 & .05 & .02 & .05 & .22 \\
\hline 4-Ethylphenol & $<.01$ & $<.01$ & $<.01$ & $<.01$ & $<.01$ & $<.01$ & $<.01$ & $<.01$ & $<.01$ & $<.01$ & $<.01$ \\
\hline 5-Methyl-1H-benzotriazole & $<.01$ & .04 & .01 & $<.01$ & $<.01$ & $<.01$ & $<.01$ & $<.01$ & $<.01$ & $<.01$ & $<.01$ \\
\hline 4-Methylphenol & .03 & .02 & $<.01$ & .02 & .02 & .01 & .02 & .02 & .02 & .04 & .01 \\
\hline
\end{tabular}


Table 9. Summary of neutral organic compound data for water samples collected from 11 Minnesota lakes during 2008.—Continued

[avg, average; $\mu \mathrm{g} / \mathrm{L}$, microgram per liter; <, less than; Dup, duplicate sample; MS, matrix spike recovery in percent]

\begin{tabular}{|c|c|c|c|c|c|c|c|c|c|c|c|}
\hline Compound & $\begin{array}{c}\text { Owasso } \\
\mu \mathrm{g} / \mathrm{L}\end{array}$ & $\begin{array}{l}\text { Cedar } \\
\mu \mathrm{g} / \mathrm{L}\end{array}$ & $\begin{array}{l}\text { Budd } \\
\mu \mathrm{g} / \mathrm{L}\end{array}$ & $\begin{array}{c}\text { Sullivan } \\
\mu \mathrm{g} / \mathrm{L}\end{array}$ & $\begin{array}{c}\text { Red Sand } \\
\text { (avg) } \\
\mu \mathrm{g} / \mathrm{L}\end{array}$ & $\begin{array}{c}\text { Shingobee } \\
\mu \mathrm{g} / \mathrm{L}\end{array}$ & $\begin{array}{l}\text { White Sand } \\
\mu \mathrm{g} / \mathrm{L}\end{array}$ & $\begin{array}{c}\text { Stewart } \\
\mu \mathrm{g} / \mathrm{L}\end{array}$ & $\begin{array}{c}\text { Kabetogama } \\
\text { (avg) } \\
\mu g / L\end{array}$ & $\begin{array}{c}\text { Northern } \\
\text { Light } \\
\text { (avg) } \\
\mu \mathrm{g} / \mathrm{L}\end{array}$ & $\begin{array}{c}\text { Elk } \\
\text { (avg) } \\
\mu \mathrm{g} / \mathrm{L}\end{array}$ \\
\hline 4-Nonylphenol & $<.05$ & $<.05$ & $<.05$ & $<.05$ & $<.05$ & .11 & $<.05$ & $<.05$ & $<.05$ & .21 & $<.05$ \\
\hline 4-Nonylphenolmonoethoxylate & $<.05$ & $<.05$ & .06 & $<.05$ & $<.05$ & .09 & $<.05$ & $<.05$ & $<.05$ & .17 & $<.05$ \\
\hline 4-Nonylphenoldiethoxylate & $<.05$ & $<.05$ & $<.05$ & $<.05$ & $<.05$ & .17 & $<.05$ & $<.05$ & $<.05$ & .13 & $<.05$ \\
\hline 4-Nonylphenoltriethoxylate & $<.05$ & $<.05$ & $<.05$ & $<.05$ & $<.05$ & .12 & $<.05$ & $<.05$ & $<.05$ & $<.05$ & $<.05$ \\
\hline 4-Nonylphenoltetraethoxylate & $<.05$ & $<.05$ & $<.05$ & $<.05$ & $<.05$ & .03 & $<.05$ & $<.05$ & $<.05$ & $<.05$ & $<.05$ \\
\hline 4-normal-Octylphenol & $<.01$ & $<.01$ & $<.01$ & $<.01$ & $<.01$ & $<.01$ & $<.01$ & $<.01$ & $<.01$ & $<.01$ & $<.01$ \\
\hline 4-tert-Octylphenol & $<.01$ & $<.01$ & $<.01$ & $<.01$ & $<.01$ & $<.01$ & $<.01$ & $<.01$ & $<.01$ & .01 & $<.01$ \\
\hline 4-tert-Octylphenolmonoethoxylate & $<.01$ & $<.01$ & $<.01$ & $<.01$ & $<.01$ & $<.01$ & $<.01$ & $<.01$ & $<.01$ & $<.01$ & $<.01$ \\
\hline 4-tert-Octylphenoldiethoxylate & $<.01$ & $<.01$ & .03 & $<.01$ & $<.01$ & .03 & $<.01$ & $<.01$ & $<.01$ & .04 & $<.01$ \\
\hline 4-tert-Octylphenoltriethoxylate & $<.01$ & $<.01$ & $<.01$ & $<.01$ & $<.01$ & .01 & $<.01$ & $<.01$ & $<.01$ & $<.01$ & $<.01$ \\
\hline 4-tert-Octylphenoltetraethoxylate & $<.01$ & $<.01$ & $<.01$ & $<.01$ & $<.01$ & $<.01$ & $<.01$ & $<.01$ & $<.01$ & $<.01$ & $<.01$ \\
\hline 4-tert-Octylphenolpentaethoxylate & $<.01$ & $<.01$ & $<.01$ & $<.01$ & $<.01$ & $<.01$ & $<.01$ & $<.01$ & $<.01$ & $<.01$ & $<.01$ \\
\hline 4-tert-Pentylphenol & $<.01$ & $<.01$ & $<.01$ & $<.01$ & $<.01$ & $<.01$ & $<.01$ & $<.01$ & $<.01$ & $<.01$ & $<.01$ \\
\hline 4-Propylphenol & $<.01$ & $<.01$ & $<.01$ & $<.01$ & $<.01$ & $<.01$ & $<.01$ & $<.01$ & $<.01$ & $<.01$ & $<.01$ \\
\hline Triclosan & $<.01$ & $<.01$ & $<.01$ & $<.01$ & $<.01$ & $<.01$ & $<.01$ & $<.01$ & $<.01$ & .01 & $<.01$ \\
\hline \multicolumn{12}{|l|}{ Surrogate Recovery (percent) } \\
\hline d6-Bisphenol A & 67 & 60 & 63 & 43 & 44 & 65 & 63 & 77 & 63 & 99 & 45 \\
\hline $\begin{array}{l}\text { d21-2,6-Di-tert-butyl-4- } \\
\text { methylphenol }\end{array}$ & 27 & 24 & 7 & 10 & 26 & 36 & 25 & 42 & 40 & 55 & 18 \\
\hline 4-normal-Nonylphenol & 43 & 30 & 38 & 32 & 34 & 42 & 32 & 49 & 50 & 71 & 37 \\
\hline \multicolumn{12}{|l|}{ 4-normal- } \\
\hline Nonylphenolmonoethoxylate & 22 & 17 & 24 & 28 & 21 & 36 & 20 & 31 & 34 & 46 & 27 \\
\hline 4-normal-Nonylphenoldiethoxylate & 17 & 13 & 22 & 21 & 17 & 24 & 14 & 14 & 22 & 22 & 20 \\
\hline
\end{tabular}


Duplicates, Blanks, and Matrix Spikes

Table 9. Summary of neutral organic compound data for water samples collected from 11 Minnesota lakes during 2008.-Continued

[avg, average; $\mu \mathrm{g} / \mathrm{L}$, microgram per liter; $<$, less than; Dup, duplicate sample; MS, matrix spike recovery in percent]

\begin{tabular}{|c|c|c|c|c|c|c|c|c|c|c|c|c|c|}
\hline Compound & $\begin{array}{l}\text { Red } \\
\text { Sand } \\
\mu \mathrm{g} / \mathrm{L}\end{array}$ & $\begin{array}{l}\text { Red } \\
\text { Sand } \\
\text { Dup } \\
\mu \mathrm{g} / \mathrm{L}\end{array}$ & $\begin{array}{c}\text { Elk } \\
\mu \mathrm{g} / \mathrm{L}\end{array}$ & $\begin{array}{c}\text { Elk Dup } \\
\mu \mathrm{g} / \mathrm{L}\end{array}$ & $\begin{array}{c}\text { Kabetogama } \\
\mu \mathrm{g} / \mathrm{L}\end{array}$ & $\begin{array}{c}\text { Kabetogama } \\
\text { Dup } \\
\mu \mathrm{g} / \mathrm{L}\end{array}$ & $\begin{array}{c}\text { Northern } \\
\text { Light } \\
\mu \mathrm{g} / \mathrm{L}\end{array}$ & $\begin{array}{c}\text { Northern } \\
\text { Light } \\
\text { Dup } \\
\mu \mathrm{g} / \mathrm{L}\end{array}$ & $\begin{array}{c}\text { Distilled } \\
\text { Water } \\
\text { Blank } \\
\mu \mathrm{g} / \mathrm{L}\end{array}$ & $\begin{array}{c}\text { Distilled } \\
\text { Water } \\
\text { Blank } \\
\mu \mathrm{g} / \mathrm{L}\end{array}$ & $\begin{array}{c}\text { Field } \\
\text { Blank } \\
\mu \mathrm{g} / \mathrm{L}\end{array}$ & $\begin{array}{c}\text { Budd } \\
\text { MS } \\
\text { percent }\end{array}$ & $\begin{array}{c}\text { White } \\
\text { Sand } \\
\text { MS } \\
\text { percent }\end{array}$ \\
\hline Bisphenol A & $<0.01$ & $<0.01$ & $<0.01$ & $<0.01$ & $<0.01$ & $<0.01$ & 0.01 & 0.01 & $<0.01$ & $<0.01$ & $<0.01$ & 34 & 72 \\
\hline 2[3]-tert-Butyl-4-methylphenol & $<.01$ & $<.01$ & $<.01$ & $<.01$ & $<.01$ & $<.01$ & $<.01$ & $<.01$ & $<.01$ & $<.01$ & $<.01$ & 0 & 27 \\
\hline 4-tert-Butylphenol & $<.01$ & $<.01$ & $<.01$ & $<.01$ & $<.01$ & $<.01$ & $<.01$ & $<.01$ & $<.01$ & $<.01$ & $<.01$ & 29 & 60 \\
\hline Caffeine & $<.01$ & $<.01$ & .01 & $<.01$ & $<.01$ & $<.01$ & .03 & .01 & $<.01$ & .02 & $<.01$ & 41 & 64 \\
\hline 1,2-Dichlorobenzene & $<.01$ & $<.01$ & $<.01$ & $<.01$ & $<.01$ & $<.01$ & $<.01$ & $<.01$ & $<.01$ & $<.01$ & $<.01$ & 27 & 59 \\
\hline 1,3-Dichlorobenzene & $<.01$ & $<.01$ & $<.01$ & $<.01$ & $<.01$ & $<.01$ & $<.01$ & $<.01$ & $<.01$ & $<.01$ & $<.01$ & 26 & 57 \\
\hline 1,4-Dichlorobenzene & $<.01$ & $<.01$ & $<.01$ & $<.01$ & $<.01$ & $<.01$ & $<.01$ & $<.01$ & $<.01$ & $<.01$ & $<.01$ & 26 & 57 \\
\hline 2,6-Di-tert-butyl-1,4-benzoquinone & .08 & $<.01$ & .15 & $<.01$ & $<.01$ & $<.01$ & $<.01$ & $<.01$ & .07 & $<.01$ & $<.01$ & 33 & 61 \\
\hline 2,6-Di-tert-butyl-4-methylphenol & .01 & .02 & .02 & .01 & $<.01$ & $<.01$ & .02 & $<.01$ & .01 & $<.01$ & $<.01$ & 8 & 36 \\
\hline 2,6-Di-tert-butylphenol & $<.01$ & $<.01$ & $<.01$ & $<.01$ & $<.01$ & $<.01$ & $<.01$ & $<.01$ & $<.01$ & $<.01$ & $<.01$ & 0 & 41 \\
\hline$N, N$-Diethyl-meta-toluamide & .01 & .01 & .27 & .17 & .02 & .02 & .07 & .03 & $<.01$ & .08 & $<.01$ & 49 & 73 \\
\hline 4-Ethylphenol & $<.01$ & $<.01$ & $<.01$ & $<.01$ & $<.01$ & $<.01$ & $<.01$ & $<.01$ & $<.01$ & $<.01$ & $<.01$ & 29 & 59 \\
\hline 5-Methyl-1H-benzotriazole & $<.01$ & $<.01$ & $<.01$ & $<.01$ & $<.01$ & $<.01$ & $<.01$ & $<.01$ & $<.01$ & $<.01$ & $<.01$ & 29 & 44 \\
\hline 4-Methylphenol & .01 & .02 & .01 & .02 & .01 & .03 & .05 & .03 & $<.01$ & $<.01$ & $<.01$ & 26 & 57 \\
\hline 4-Nonylphenol & $<.05$ & $<.05$ & $<.05$ & $<.05$ & $<.05$ & $<.05$ & .28 & .15 & $<.05$ & $<.05$ & $<.05$ & 15 & 59 \\
\hline 4-Nonylphenolmonoethoxylate & $<.05$ & $<.05$ & $<.05$ & $<.05$ & $<.05$ & $<.05$ & .17 & $<.05$ & $<.05$ & $<.05$ & $<.05$ & 17 & 56 \\
\hline 4-Nonylphenoldiethoxylate & $<.05$ & $<.05$ & $<.05$ & $<.05$ & $<.05$ & $<.05$ & $<.05$ & .13 & $<.05$ & $<.05$ & $<.05$ & 14 & 52 \\
\hline 4-Nonylphenoltriethoxylate & $<.05$ & $<.05$ & $<.05$ & $<.05$ & $<.05$ & $<.05$ & $<.05$ & $<.05$ & $<.05$ & $<.05$ & $<.05$ & 12 & 43 \\
\hline 4-Nonylphenoltetraethoxylate & $<.05$ & $<.05$ & $<.05$ & $<.05$ & $<.05$ & $<.05$ & $<.05$ & $<.05$ & $<.05$ & $<.05$ & $<.05$ & 10 & 35 \\
\hline 4-normal-Octylphenol & $<.01$ & $<.01$ & $<.01$ & $<.01$ & $<.01$ & $<.01$ & $<.01$ & $<.01$ & $<.01$ & $<.01$ & $<.01$ & 18 & 58 \\
\hline 4-tert-Octylphenol & $<.01$ & $<.01$ & $<.01$ & $<.01$ & $<.01$ & $<.01$ & .01 & $<.01$ & $<.01$ & $<.01$ & $<.01$ & 23 & 71 \\
\hline 4-tert-Octylphenolmonoethoxylate & $<.01$ & $<.01$ & $<.01$ & $<.01$ & $<.01$ & $<.01$ & $<.01$ & $<.01$ & $<.01$ & $<.01$ & $<.01$ & 25 & 67 \\
\hline 4-tert-Octylphenoldiethoxylate & $<.01$ & $<.01$ & $<.01$ & $<.01$ & $<.01$ & $<.01$ & .04 & .04 & $<.01$ & $<.01$ & $<.01$ & 17 & 56 \\
\hline 4-tert-Octylphenoltriethoxylate & $<.01$ & $<.01$ & $<.01$ & $<.01$ & $<.01$ & $<.01$ & $<.01$ & $<.01$ & $<.01$ & $<.01$ & $<.01$ & 14 & 44 \\
\hline 4-tert-Octylphenoltetraethoxylate & $<.01$ & $<.01$ & $<.01$ & $<.01$ & $<.01$ & $<.01$ & $<.01$ & $<.01$ & $<.01$ & $<.01$ & $<.01$ & 13 & 45 \\
\hline 4-tert-Octylphenolpentaethoxylate & $<.01$ & $<.01$ & $<.01$ & $<.01$ & $<.01$ & $<.01$ & $<.01$ & $<.01$ & $<.01$ & $<.01$ & $<.01$ & 9 & 28 \\
\hline 4-tert-Pentylphenol & $<.01$ & $<.01$ & $<.01$ & $<.01$ & $<.01$ & $<.01$ & $<.01$ & $<.01$ & $<.01$ & $<.01$ & $<.01$ & 30 & 64 \\
\hline
\end{tabular}


Table 9. Summary of neutral organic compound data for water samples collected from 11 Minnesota lakes during 2008.—Continued

[avg, average; $\mu \mathrm{g} / \mathrm{L}$, microgram per liter; $<$, less than; Dup, duplicate sample; MS, matrix spike recovery in percent]

\begin{tabular}{|c|c|c|c|c|c|c|c|c|c|c|c|c|c|}
\hline Compound & $\begin{array}{l}\text { Red } \\
\text { Sand } \\
\mu \mathrm{g} / \mathrm{L}\end{array}$ & $\begin{array}{l}\text { Red } \\
\text { Sand } \\
\text { Dup } \\
\mu \mathrm{g} / \mathrm{L}\end{array}$ & $\begin{array}{l}\text { Elk } \\
\mu \mathrm{g} / \mathrm{L}\end{array}$ & $\begin{array}{c}\text { Elk Dup } \\
\mu \mathrm{g} / \mathrm{L}\end{array}$ & $\begin{array}{c}\text { Kabetogama } \\
\mu \mathrm{g} / \mathrm{L}\end{array}$ & $\begin{array}{c}\text { Kabetogama } \\
\text { Dup } \\
\mu g / L\end{array}$ & $\begin{array}{c}\text { Northern } \\
\text { Light } \\
\mu \mathrm{g} / \mathrm{L}\end{array}$ & $\begin{array}{l}\text { Northern } \\
\text { Light } \\
\text { Dup } \\
\mu \mathrm{g} / \mathrm{L}\end{array}$ & $\begin{array}{c}\text { Distilled } \\
\text { Water } \\
\text { Blank } \\
\mu \mathrm{g} / \mathrm{L}\end{array}$ & $\begin{array}{c}\text { Distilled } \\
\text { Water } \\
\text { Blank } \\
\mu \mathrm{g} / \mathrm{L}\end{array}$ & $\begin{array}{c}\text { Field } \\
\text { Blank } \\
\mu \mathrm{g} / \mathrm{L}\end{array}$ & $\begin{array}{l}\text { Budd } \\
\text { MS } \\
\text { percent }\end{array}$ & $\begin{array}{l}\text { White } \\
\text { Sand } \\
\text { MS } \\
\text { percent }\end{array}$ \\
\hline 4-Propylphenol & $<.01$ & $<.01$ & $<.01$ & $<.01$ & $<.01$ & $<.01$ & $<.01$ & $<.01$ & $<.01$ & $<.01$ & $<.01$ & 29 & 60 \\
\hline Triclosan & $<.01$ & $<.01$ & $<.01$ & $<.01$ & $<.01$ & $<.01$ & $<.01$ & .01 & $<.01$ & $<.01$ & $<.01$ & 20 & 62 \\
\hline \multicolumn{14}{|l|}{ Surrogate Recovery } \\
\hline d6-Bisphenol A & 37 & 51 & 41 & 50 & 47 & 79 & 112 & 87 & 13 & 61 & 117 & 45 & 66 \\
\hline d21-2,6-Di-tert-butyl-4-methylphenol & 24 & 28 & 18 & 19 & 30 & 50 & 62 & 48 & 32 & 28 & 67 & 5 & 34 \\
\hline 4-normal-Nonylphenol & 31 & 36 & 34 & 40 & 38 & 61 & 79 & 63 & 48 & 91 & 120 & 17 & 52 \\
\hline $\begin{array}{l}\text { 4-normal-Nonylphenolmonoethox- } \\
\text { ylate }\end{array}$ & 20 & 23 & 24 & 30 & 27 & 41 & 57 & 34 & 33 & 102 & 96 & 14 & 39 \\
\hline 4-normal-Nonylphenoldiethoxylate & 16 & 17 & 17 & 23 & 18 & 26 & 28 & 17 & 14 & 57 & 46 & 10 & 26 \\
\hline
\end{tabular}


Table 10. Summary of steroid and steroidal hormone compound data for water samples collected from 11 Minnesota lakes during 2008.

[avg, average; $\mu \mathrm{g} / \mathrm{L}$, microgram per liter; <, less than; Dup, duplicate sample; MS, matrix spike recovery in percent]

\begin{tabular}{|c|c|c|c|c|c|c|c|c|c|c|c|}
\hline Compound & $\begin{array}{c}\text { Owasso } \\
\mu \mathrm{g} / \mathrm{L}\end{array}$ & $\begin{array}{c}\text { Cedar } \\
\mu \mathrm{g} / \mathrm{L}\end{array}$ & $\begin{array}{c}\text { Budd } \\
\mu \mathrm{g} / \mathrm{L}\end{array}$ & $\begin{array}{c}\text { Sullivan } \\
\mu \mathrm{g} / \mathrm{L}\end{array}$ & $\begin{array}{c}\text { Red Sand } \\
\text { (avg) } \\
\mu \mathrm{g} / \mathrm{L}\end{array}$ & $\begin{array}{c}\text { Shingobee } \\
\mu \mathrm{g} / \mathrm{L}\end{array}$ & $\begin{array}{c}\text { White Sand } \\
\text { (avg) } \\
\mu \mathrm{g} / \mathrm{L}\end{array}$ & $\begin{array}{c}\text { Stewart } \\
\mu \mathrm{g} / \mathrm{L}\end{array}$ & $\begin{array}{c}\text { Kabetogama } \\
\text { (avg) } \\
\mu \mathrm{g} / \mathrm{L}\end{array}$ & $\begin{array}{l}\text { Northern } \\
\text { Light } \\
\text { (avg) } \\
\mu \mathrm{g} / \mathrm{L}\end{array}$ & $\begin{array}{c}\text { Elk (avg) } \\
\mu g / L\end{array}$ \\
\hline 4-Androstene-3,17-dione & $<0.0001$ & $<0.0001$ & $<0.0001$ & 0.0007 & 0.0004 & 0.0005 & 0.0010 & 0.0003 & 0.0005 & $<0.0001$ & 0.0008 \\
\hline cis-Androsterone & $<.0001$ & $<.0001$ & $<.0001$ & $<.0001$ & $<.0001$ & $<.0001$ & $<.0001$ & $<.0001$ & $<.0001$ & $<.0001$ & $<.0001$ \\
\hline Cholesterol & 9.0 & 7.8 & 2.5 & 7.1 & 5.7 & $<.005$ & 7.5 & $<.005$ & 2.0 & $<.005$ & .008 \\
\hline Coprostanol & NM & $<.005$ & $<.005$ & $<.005$ & $<.005$ & $<.005$ & $<.005$ & $<.005$ & $<.005$ & $<.005$ & $<.005$ \\
\hline Diethylstilbesterol & $<.0001$ & $<.0001$ & $<.0001$ & $<.0001$ & $<.0001$ & $<.0001$ & $<.0001$ & $<.0001$ & $<.0001$ & $<.0001$ & $<.0001$ \\
\hline Equilenin & $<.0001$ & $<.0001$ & $<.0001$ & $<.0001$ & $<.0001$ & $<.0001$ & $<.0001$ & $<.0001$ & $<.0001$ & $<.0001$ & $<.0001$ \\
\hline Equilin & $<.0001$ & $<.0001$ & $<.0001$ & $<.0001$ & $<.0001$ & $<.0001$ & .0003 & $<.0001$ & $<.0001$ & $<.0001$ & $<.0001$ \\
\hline $17 \alpha$-Ethinylestradiol & $<.0001$ & $<.0001$ & $<.0001$ & $<.0001$ & $<.0001$ & $<.0001$ & $<.0001$ & $<.0001$ & $<.0001$ & $<.0001$ & $<.0001$ \\
\hline $17 \alpha$-Estradiol & $<.0001$ & $<.0001$ & $<.0001$ & $<.0001$ & $<.0001$ & $<.0001$ & $<.0001$ & $<.0001$ & $<.0001$ & $<.0001$ & $<.0001$ \\
\hline $17 \beta$-Estradiol & .0001 & .0004 & $<.0001$ & .0001 & $<.0001$ & $<.0001$ & .0001 & $<.0001$ & $<.0001$ & .0005 & .0004 \\
\hline Estriol & $<.0001$ & $<.0001$ & $<.0001$ & $<.0001$ & $<.0001$ & $<.0001$ & $<.0001$ & $<.0001$ & $<.0001$ & $<.0001$ & $<.0001$ \\
\hline Estrone & $<.0001$ & $<.0001$ & $<.0001$ & .0007 & .0006 & .0011 & .0006 & .0015 & $<.0001$ & $<.0001$ & .0011 \\
\hline Mestranol & .0002 & $<.0001$ & $<.0001$ & $<.0001$ & $<.0001$ & $<.0001$ & $<.0001$ & $<.0001$ & $<.0001$ & $<.0001$ & $<.0001$ \\
\hline Norethindrone & $<.0001$ & $<.0001$ & $<.0001$ & $<.0001$ & $<.0001$ & $<.0001$ & $<.0001$ & $<.0001$ & $<.0001$ & $<.0001$ & $<.0001$ \\
\hline Progesterone & $<.0001$ & $<.0001$ & $<.0001$ & $<.0001$ & $<.0001$ & $<.0001$ & $<.0001$ & $<.0001$ & $<.0001$ & $<.0001$ & $<.0001$ \\
\hline Testosterone & $<.0001$ & $<.0001$ & $<.0001$ & $<.0001$ & $<.0001$ & $<.0001$ & .0005 & $<.0001$ & $<.0001$ & $<.0001$ & $<.0001$ \\
\hline dihydro-Testosterone & $<.0001$ & $<.0001$ & $<.0001$ & $<.0001$ & $<.0001$ & $<.0001$ & $<.0001$ & $<.0001$ & $<.0001$ & $<.0001$ & $<.0001$ \\
\hline epi-Testosterone & $<.0001$ & $<.0001$ & $<.0001$ & $<.0001$ & $<.0001$ & $<.0001$ & $<.0001$ & $<.0001$ & $<.0001$ & $<.0001$ & $<.0001$ \\
\hline 11-keto-Testosterone & $<.0001$ & $<.0001$ & $<.0001$ & $<.0001$ & $<.0001$ & $<.0001$ & $<.0001$ & $<.0001$ & $<.0001$ & $<.0001$ & $<.0001$ \\
\hline \multicolumn{12}{|c|}{ Surrogate Recovery (percent) } \\
\hline d7-Androstenedione & 0 & 0 & 0 & 45 & 62 & 64 & 45 & 68 & 51 & 28 & 58 \\
\hline d7-Cholesterol & 14 & 33 & 23 & 56 & 51 & 27 & 52 & 30 & 62 & 59 & 29 \\
\hline d12-Chrysene & 79 & 92 & 82 & 88 & 84 & 76 & 86 & 84 & 98 & 89 & 83 \\
\hline d4-Dihydrotestosterone & 0 & 0 & 0 & 62 & 86 & 79 & 28 & 81 & 31 & 27 & 55 \\
\hline d4-Ethinyl estradiol & 49 & 60 & 57 & 82 & 81 & 53 & 84 & 74 & 91 & 86 & 41 \\
\hline d4-Estradiol & 50 & 65 & 55 & 80 & 74 & 52 & 61 & 81 & 71 & 80 & 43 \\
\hline d3-Estriol & 41 & 53 & 36 & 60 & 50 & 19 & 56 & 40 & 70 & 57 & 44 \\
\hline d4-Estrone & 0 & 0 & 0 & 85 & 75 & 59 & 98 & 88 & 103 & 102 & 43 \\
\hline d4-Mestranol & 56 & 63 & 59 & 89 & 93 & 64 & 89 & 79 & 90 & 85 & 40 \\
\hline
\end{tabular}


Table 10. Summary of steroid and steroidal hormone compound data for water samples collected from 11 Minnesota lakes during 2008.—Continued

[avg, average; $\mu \mathrm{g} / \mathrm{L}$, microgram per liter; <, less than; Dup, duplicate sample; MS, matrix spike recovery in percent]

\begin{tabular}{|c|c|c|c|c|c|c|c|c|c|c|c|}
\hline Compound & $\begin{array}{c}\text { Owasso } \\
\mu \mathrm{g} / \mathrm{L}\end{array}$ & $\begin{array}{c}\text { Cedar } \\
\mu \mathrm{g} / \mathrm{L}\end{array}$ & $\begin{array}{l}\text { Budd } \\
\mu \mathrm{g} / \mathrm{L}\end{array}$ & $\begin{array}{c}\text { Sullivan } \\
\mu \mathrm{g} / \mathrm{L}\end{array}$ & $\begin{array}{c}\text { Red Sand } \\
\text { (avg) } \\
\mu g / L\end{array}$ & $\begin{array}{c}\text { Shingobee } \\
\mu \mathrm{g} / \mathrm{L}\end{array}$ & $\begin{array}{c}\text { White Sand } \\
\text { (avg) } \\
\mu \mathrm{g} / \mathrm{L}\end{array}$ & $\begin{array}{c}\text { Stewart } \\
\mu \mathrm{g} / \mathrm{L}\end{array}$ & $\begin{array}{c}\text { Kabetogama } \\
\text { (avg) } \\
\mu \mathrm{g} / \mathrm{L}\end{array}$ & $\begin{array}{l}\text { Northern } \\
\text { Light } \\
\text { (avg) } \\
\mu \mathrm{g} / \mathrm{L}\end{array}$ & $\begin{array}{c}\text { Elk (avg) } \\
\mu \mathrm{g} / \mathrm{L}\end{array}$ \\
\hline d6-Norethindrone & 0 & 0 & 0 & 35 & 57 & 58 & 57 & 79 & 80 & 77 & 43 \\
\hline d9-Progesterone & 0 & 0 & 0 & 0 & 2 & 19 & 0 & 19 & 1 & 4 & 1 \\
\hline d5-Testosterone & 0 & 0 & 0 & 47 & 69 & 67 & 43 & 66 & 39 & 30 & 54 \\
\hline
\end{tabular}

Duplicates, Blanks, and Matrix Spikes

\begin{tabular}{|c|c|c|c|c|c|c|c|c|c|c|c|c|c|c|c|}
\hline Compound & $\begin{array}{l}\text { Red } \\
\text { Sand } \\
\mu \mathrm{g} / \mathrm{L}\end{array}$ & $\begin{array}{l}\text { Red } \\
\text { Sand } \\
\text { Dup } \\
\mu \mathrm{g} / \mathrm{L}\end{array}$ & $\begin{array}{l}\text { White } \\
\text { Sand } \\
\mu \mathrm{g} / \mathrm{L}\end{array}$ & $\begin{array}{l}\text { White } \\
\text { Sand } \\
\text { Dup } \\
\mu \mathrm{g} / \mathrm{L}\end{array}$ & $\begin{array}{c}\text { Kabetogama } \\
\mu \mathrm{g} / \mathrm{L}\end{array}$ & $\begin{array}{c}\text { Kabetogama } \\
\text { Dup } \\
\mu \mathrm{g} / \mathrm{L}\end{array}$ & $\begin{array}{c}\text { Northern } \\
\text { Light } \\
\mu \mathrm{g} / \mathrm{L}\end{array}$ & $\begin{array}{l}\text { Northern } \\
\text { Light } \\
\text { Dup } \\
\mu \mathrm{g} / \mathrm{L}\end{array}$ & $\begin{array}{l}\text { Elk } \\
\mu g / L\end{array}$ & $\begin{array}{c}\text { Elk Dup } \\
\mu \mathrm{g} / \mathrm{L}\end{array}$ & $\begin{array}{c}\text { Field } \\
\text { blank } \\
\mu \mathrm{g} / \mathrm{L}\end{array}$ & $\begin{array}{c}\text { Distilled } \\
\text { Water } \\
\text { Blank } \\
\mu \mathrm{g} / \mathrm{L}\end{array}$ & $\begin{array}{c}\text { Distilled } \\
\text { Water } \\
\text { Blank } \\
\mu \mathrm{g} / \mathrm{L}\end{array}$ & $\begin{array}{l}\text { Sullivan } \\
\text { MS } \\
\text { percent }\end{array}$ & $\begin{array}{c}\text { Distilled } \\
\text { Water } \\
\text { MS } \\
\text { percent }\end{array}$ \\
\hline 4-Androstene-3,17-dione & $<.0001$ & .0004 & .0010 & .0010 & .0006 & .0004 & $<.0001$ & $<.0001$ & .0008 & .0009 & $<.0001$ & $<.0001$ & $<.0001$ & 92 & 84 \\
\hline cis-Androsterone & $<.0001$ & $<.0001$ & $<.0001$ & $<.0001$ & $<.0001$ & $<.0001$ & $<.0001$ & $<.0001$ & $<.0001$ & $<.0001$ & $<.0001$ & $<.0001$ & $<.0001$ & 99 & 108 \\
\hline Cholesterol & 6.5 & 4.8 & 7.5 & 7.5 & 2.0 & 2.0 & $<.005$ & $<.005$ & 8.5 & 8.1 & $<.005$ & $<.005$ & $<.005$ & 71 & 145 \\
\hline Coprostanol & $<.005$ & $<.005$ & $<.005$ & $<.005$ & $<.005$ & $<.005$ & $<.005$ & $<.005$ & $<.005$ & $<.005$ & $<.005$ & $<.005$ & $<.005$ & 98 & 154 \\
\hline Diethylstilbesterol & $<.0001$ & $<.0001$ & $<.0001$ & $<.0001$ & $<.0001$ & $<.0001$ & $<.0001$ & $<.0001$ & $<.0001$ & $<.0001$ & $<.0001$ & $<.0001$ & $<.0001$ & 81 & 99 \\
\hline Equilenin & $<.0001$ & $<.0001$ & $<.0001$ & $<.0001$ & $<.0001$ & $<.0001$ & $<.0001$ & $<.0001$ & $<.0001$ & $<.0001$ & $<.0001$ & $<.0001$ & $<.0001$ & 34 & 23 \\
\hline Equilin & $<.0001$ & $<.0001$ & $<.0001$ & .0003 & $<.0001$ & $<.0001$ & $<.0001$ & $<.0001$ & $<.0001$ & $<.0001$ & $<.0001$ & $<.0001$ & $<.0001$ & 38 & 4 \\
\hline $17 \alpha$-Ethinylestradiol & $<.0001$ & $<.0001$ & $<.0001$ & $<.0001$ & $<.0001$ & $<.0001$ & $<.0001$ & $<.0001$ & $<.0001$ & $<.0001$ & $<.0001$ & $<.0001$ & $<.0001$ & 97 & 98 \\
\hline $17 \alpha$-Estradiol & $<.0001$ & $<.0001$ & $<.0001$ & $<.0001$ & $<.0001$ & $<.0001$ & $<.0001$ & $<.0001$ & $<.0001$ & $<.0001$ & $<.0001$ & $<.0001$ & $<.0001$ & 114 & 99 \\
\hline $17 \beta$-Estradiol & $<.0001$ & $<.0001$ & .0001 & .0001 & $<.0001$ & $<.0001$ & .0008 & .0001 & .0004 & .0004 & $<.0001$ & $<.0001$ & $<.0001$ & 102 & 97 \\
\hline Estriol & $<.0001$ & $<.0001$ & $<.0001$ & $<.0001$ & $<.0001$ & $<.0001$ & $<.0001$ & $<.0001$ & $<.0001$ & $<.0001$ & $<.0001$ & $<.0001$ & $<.0001$ & 98 & 97 \\
\hline Estrone & $<.0001$ & .0007 & .0006 & .0007 & $<.0001$ & $<.0001$ & $<.0001$ & $<.0001$ & .0011 & .0011 & $<.0001$ & $<.0001$ & $<.0001$ & 90 & 79 \\
\hline Mestranol & $<.0001$ & $<.0001$ & $<.0001$ & $<.0001$ & $<.0001$ & $<.0001$ & $<.0001$ & $<.0001$ & $<.0001$ & $<.0001$ & $<.0001$ & $<.0001$ & $<.0001$ & 101 & 101 \\
\hline Norethindrone & $<.0001$ & $<.0001$ & $<.0001$ & $<.0001$ & $<.0001$ & $<.0001$ & $<.0001$ & $<.0001$ & $<.0001$ & $<.0001$ & $<.0001$ & $<.0001$ & $<.0001$ & 97 & 94 \\
\hline
\end{tabular}


Table 10. Summary of steroid and steroidal hormone compound data for water samples collected from 11 Minnesota lakes during 2008.—Continued

$[\mathrm{avg}$, average; $\mu \mathrm{g} / \mathrm{L}$, microgram per liter; <, less than; Dup, duplicate sample; MS, matrix spike recovery in percent]

\begin{tabular}{|c|c|c|c|c|c|c|c|c|c|c|c|c|c|c|c|}
\hline Compound & $\begin{array}{l}\text { Red } \\
\text { Sand } \\
\mu \mathrm{g} / \mathrm{L}\end{array}$ & $\begin{array}{l}\text { Red } \\
\text { Sand } \\
\text { Dup } \\
\mu \mathrm{g} / \mathrm{L}\end{array}$ & $\begin{array}{l}\text { White } \\
\text { Sand } \\
\mu \mathrm{g} / \mathrm{L}\end{array}$ & $\begin{array}{l}\text { White } \\
\text { Sand } \\
\text { Dup } \\
\mu \mathrm{g} / \mathrm{L}\end{array}$ & $\begin{array}{c}\text { Kabetogama } \\
\mu \mathrm{g} / \mathrm{L}\end{array}$ & $\begin{array}{c}\text { Kabetogama } \\
\text { Dup } \\
\mu \mathrm{g} / \mathrm{L}\end{array}$ & $\begin{array}{c}\text { Northern } \\
\text { Light } \\
\mu \mathrm{g} / \mathrm{L}\end{array}$ & $\begin{array}{l}\text { Northern } \\
\text { Light } \\
\text { Dup } \\
\mu \mathrm{g} / \mathrm{L}\end{array}$ & $\begin{array}{l}\text { Elk } \\
\mu g / L\end{array}$ & $\begin{array}{c}\text { Elk Dup } \\
\mu \mathrm{g} / \mathrm{L}\end{array}$ & $\begin{array}{c}\text { Field } \\
\text { blank } \\
\mu \mathrm{g} / \mathrm{L}\end{array}$ & $\begin{array}{c}\text { Distilled } \\
\text { Water } \\
\text { Blank } \\
\mu \mathrm{g} / \mathrm{L}\end{array}$ & $\begin{array}{c}\text { Distilled } \\
\text { Water } \\
\text { Blank } \\
\mu \mathrm{g} / \mathrm{L}\end{array}$ & $\begin{array}{l}\text { Sullivan } \\
\text { MS } \\
\text { percent }\end{array}$ & $\begin{array}{c}\text { Distilled } \\
\text { Water } \\
\text { MS } \\
\text { percent }\end{array}$ \\
\hline Progesterone & $<.0001$ & $<.0001$ & $<.0001$ & $<.0001$ & $<.0001$ & $<.0001$ & $<.0001$ & $<.0001$ & $<.0001$ & $<.0001$ & $<.0001$ & $<.0001$ & $<.0001$ & 0 & 88 \\
\hline Testosterone & $<.0001$ & $<.0001$ & $<.0001$ & .0005 & $<.0001$ & $<.0001$ & $<.0001$ & $<.0001$ & $<.0001$ & $<.0001$ & $<.0001$ & $<.0001$ & $<.0001$ & 92 & 88 \\
\hline dihydro-Testosterone & $<.0001$ & $<.0001$ & $<.0001$ & $<.0001$ & $<.0001$ & $<.0001$ & $<.0001$ & $<.0001$ & $<.0001$ & $<.0001$ & $<.0001$ & $<.0001$ & $<.0001$ & & \\
\hline epi-Testosterone & $<.0001$ & $<.0001$ & $<.0001$ & $<.0001$ & $<.0001$ & $<.0001$ & $<.0001$ & $<.0001$ & $<.0001$ & $<.0001$ & $<.0001$ & $<.0001$ & $<.0001$ & 108 & 92 \\
\hline 11-keto-Testosterone & $<.0001$ & $<.0001$ & $<.0001$ & $<.0001$ & $<.0001$ & $<.0001$ & $<.0001$ & $<.0001$ & $<.0001$ & $<.0001$ & $<.0001$ & $<.0001$ & $<.0001$ & 52 & 89 \\
\hline \multicolumn{16}{|c|}{ Surrogate Recovery (percent) } \\
\hline d7-Androstenedione & 66 & 58 & 46 & 43 & 51 & 51 & 28 & 28 & 38 & 79 & 112 & 123 & 84 & 49 & 104 \\
\hline d7-Cholesterol & 56 & 46 & 62 & 43 & 58 & 67 & 96 & 22 & 13 & 45 & 12 & 19 & 11 & 31 & 8 \\
\hline d12-Chrysene & 88 & 79 & 92 & 80 & 99 & 97 & 94 & 85 & 74 & 92 & 95 & 99 & 118 & 76 & 87 \\
\hline d4-Dihydrotestosterone & 77 & 94 & 31 & 25 & 31 & 31 & 29 & 25 & 36 & 73 & 85 & 80 & 76 & 67 & 70 \\
\hline d4-Ethinyl estradiol & 85 & 77 & 91 & 77 & 91 & 91 & 104 & 68 & 29 & 54 & 82 & 74 & 73 & 63 & 51 \\
\hline d4-Estradiol & 76 & 72 & 65 & 57 & 71 & 72 & 94 & 66 & 32 & 55 & 88 & 65 & 66 & 62 & 48 \\
\hline d3-Estriol & 53 & 48 & 66 & 47 & 69 & 70 & 74 & 40 & 36 & 52 & 50 & 50 & 47 & 38 & 33 \\
\hline d4-Estrone & 83 & 68 & 104 & 93 & 103 & 102 & 117 & 87 & 33 & 52 & 98 & 81 & 79 & 77 & 64 \\
\hline d4-Mestranol & 99 & 87 & 93 & 84 & 90 & 91 & 102 & 69 & 29 & 51 & 89 & 88 & 75 & 80 & 66 \\
\hline d6-Norethindrone & 53 & 61 & 62 & 53 & 78 & 82 & 82 & 71 & 23 & 62 & 91 & 108 & 94 & 46 & 83 \\
\hline d9-Progesterone & 1 & 3 & 0 & 1 & 1 & 2 & 1 & 8 & 0 & 2 & 28 & 100 & 88 & 1 & 58 \\
\hline d5-Testosterone & 63 & 75 & 47 & 39 & 37 & 41 & 31 & 29 & 36 & 72 & 97 & 103 & 78 & 54 & 84 \\
\hline
\end{tabular}


Table 11. Summary of neutral organic compound data for sediment samples collected from 11 Minnesota lakes during 2008.

[avg, average; $\mu \mathrm{g} / \mathrm{kg}$, microgram per kilogram; <, less than; MS, matrix spike recovery in percent; Dup, duplicate sample; Trip, triplicate sample]

\begin{tabular}{|c|c|c|c|c|c|c|c|c|c|c|c|}
\hline Compound & $\begin{array}{c}\text { Owasso } \\
\mu \mathrm{g} / \mathrm{kg}\end{array}$ & $\begin{array}{l}\text { Cedar } \\
\text { (avg) } \\
\mu \mathrm{g} / \mathrm{kg}\end{array}$ & $\begin{array}{l}\text { Budd } \\
\mu \mathrm{g} / \mathrm{kg}\end{array}$ & $\begin{array}{c}\text { Sullivan } \\
\mu \mathrm{g} / \mathrm{kg}\end{array}$ & $\begin{array}{c}\text { Red Sand } \\
\text { (avg) } \\
\mu \mathrm{g} / \mathrm{kg}\end{array}$ & $\begin{array}{c}\text { Shingobee } \\
\text { (avg) } \\
\mu \mathrm{g} / \mathbf{k g}\end{array}$ & $\begin{array}{c}\text { White } \\
\text { Sand } \\
\mu \mathrm{g} / \mathrm{kg}\end{array}$ & $\begin{array}{c}\text { Stewart } \\
\mu \mathrm{g} / \mathrm{kg}\end{array}$ & $\begin{array}{c}\text { Kabe- } \\
\text { togama } \\
\mu \mathrm{g} / \mathrm{kg}\end{array}$ & $\begin{array}{c}\text { Northern } \\
\text { Light } \\
\mu \mathrm{g} / \mathrm{kg}\end{array}$ & $\begin{array}{c}\text { Elk } \\
\mu \mathrm{g} / \mathrm{kg}\end{array}$ \\
\hline Bisphenol A & 20 & 6.3 & 17 & 6.7 & 17 & 19 & 35 & $<1$ & 16 & 3.1 & 7.7 \\
\hline 2[3]-tert-Butyl-4-methylphenol & $<1$ & $<1$ & $<1$ & $<1$ & $<1$ & $<1$ & $<1$ & $<1$ & $<1$ & $<1$ & $<1$ \\
\hline 4-tert-Butylphenol & $<1$ & $<1$ & $<1$ & 6 & $<1$ & $<1$ & $<1$ & $<1$ & $<1$ & $<1$ & $<1$ \\
\hline Caffeine & $<1$ & 110 & $<1$ & $<1$ & $<1$ & $<1$ & $<1$ & $<1$ & $<1$ & $<1$ & $<1$ \\
\hline 1,2-Dichlorobenzene & $<1$ & $<1$ & $<1$ & $<1$ & $<1$ & $<1$ & $<1$ & $<1$ & $<1$ & $<1$ & $<1$ \\
\hline 1,3-Dichlorobenzene & $<1$ & $<1$ & $<1$ & $<1$ & $<1$ & $<1$ & $<1$ & $<1$ & $<1$ & $<1$ & $<1$ \\
\hline 1,4-Dichlorobenzene & $<1$ & $<1$ & $<1$ & $<1$ & 1.1 & 0 & $<1$ & $<1$ & $<1$ & 1 & $<1$ \\
\hline 2,6-Di-tert-butyl-1,4-benzoquinone & $<200$ & $<200$ & $<200$ & $<200$ & $<200$ & $<200$ & $<200$ & $<200$ & $<200$ & $<200$ & $<200$ \\
\hline 2,6-Di-tert-butyl-4-methylphenol & $<3$ & $<3$ & $<3$ & $<3$ & $<3$ & $<3$ & $<3$ & $<3$ & $<3$ & 4 & $<3$ \\
\hline 2,6-Di-tert-butylphenol & $<1$ & $<1$ & $<1$ & $<1$ & $<1$ & $<1$ & $<1$ & $<1$ & $<1$ & $<1$ & $<1$ \\
\hline$N, N$-Diethyl-meta-toluamide & $<1$ & $<1$ & $<1$ & $<1$ & $<1$ & $<1$ & $<1$ & $<1$ & $<1$ & $<1$ & $<1$ \\
\hline 4-Ethylphenol & 59 & 8.2 & $<3$ & 21 & $<3$ & 96 & 23 & $<3$ & 607 & 34 & 18 \\
\hline 5-methyl-1H-Benzotriazole & $<1$ & $<1$ & $<1$ & $<1$ & $<1$ & $<1$ & $<1$ & $<1$ & $<1$ & $<1$ & $<1$ \\
\hline 4-Methylphenol & 595 & 12 & $<3$ & 131 & 355 & 160 & 186 & $<3$ & 1,100 & 128 & 34 \\
\hline 4-Nonylphenol & $<100$ & $<100$ & $<100$ & $<100$ & 100 & 350 & $<100$ & $<100$ & $<100$ & $<100$ & $<100$ \\
\hline 4-Nonylphenolmonoethoxylate & $<20$ & $<20$ & $<20$ & $<20$ & $<20$ & 30 & $<20$ & $<20$ & $<20$ & $<20$ & $<20$ \\
\hline 4-Nonylphenoldiethoxylate & $<1$ & $<1$ & $<1$ & $<1$ & $<1$ & 59 & $<1$ & $<1$ & $<1$ & $<1$ & $<1$ \\
\hline 4-Nonylphenoltriethoxylate & $<1$ & $<1$ & $<1$ & 62 & $<1$ & $<1$ & $<1$ & $<1$ & $<1$ & $<1$ & $<1$ \\
\hline 4-Nonylphenoltetraethoxylate & $<1$ & $<1$ & $<1$ & $<1$ & $<1$ & $<1$ & $<1$ & $<1$ & $<1$ & $<1$ & $<1$ \\
\hline 4-normal-Octylphenol & $<1$ & $<1$ & $<1$ & $<1$ & $<1$ & $<1$ & $<1$ & $<1$ & $<1$ & $<1$ & $<1$ \\
\hline 4-tert-Octylphenol & 98 & 4.4 & $<3$ & 8 & $<3$ & 22 & 33 & $<3$ & $<3$ & $<3$ & 5 \\
\hline 4-tert-Octylphenolmonoethoxylate & $<1$ & $<1$ & $<1$ & $<1$ & 1.3 & 6 & $<1$ & $<1$ & $<1$ & $<1$ & $<1$ \\
\hline 4-tert-Octylphenoldiethoxylate & $<1$ & $<1$ & $<1$ & $<1$ & 21 & $<1$ & 74 & 3 & 45 & $<1$ & $<1$ \\
\hline 4-tert-Octylphenoltriethoxylate & $<1$ & $<1$ & $<1$ & $<1$ & $<1$ & $<1$ & $<1$ & $<1$ & $<1$ & $<1$ & $<1$ \\
\hline 4-tert-Octylphenoltetraethoxylate & $<1$ & $<1$ & $<1$ & $<1$ & $<1$ & $<1$ & $<1$ & $<1$ & $<1$ & $<1$ & $<1$ \\
\hline 4-tert-Octylphenolpentaethoxylate & $<1$ & $<1$ & $<1$ & $<1$ & $<1$ & $<1$ & $<1$ & $<1$ & $<1$ & $<1$ & $<1$ \\
\hline 4-tert-Pentylphenol & 88 & $<1$ & $<1$ & 23 & $<1$ & $<1$ & 74 & $<1$ & $<1$ & $<1$ & $<1$ \\
\hline 4-Propylphenol & $<1$ & $<1$ & $<1$ & $<1$ & $<1$ & $<1$ & $<1$ & $<1$ & $<1$ & $<1$ & $<1$ \\
\hline Triclosan & 33 & 44 & $<1$ & $<1$ & $<1$ & $<1$ & $<1$ & $<1$ & $<1$ & $<1$ & $<1$ \\
\hline
\end{tabular}


Table 11. Summary of neutral organic compound data for sediment samples collected from 11 Minnesota lakes during 2008.-Continued

[avg, average; $\mu \mathrm{g} / \mathrm{kg}$, microgram per kilogram; <, less than; MS, matrix spike recovery in percent; Dup, duplicate sample; Trip, triplicate sample]

\begin{tabular}{|c|c|c|c|c|c|c|c|c|c|c|c|}
\hline Compound & $\begin{array}{c}\text { Owasso } \\
\mu \mathrm{g} / \mathrm{kg}\end{array}$ & $\begin{array}{c}\text { Cedar } \\
\text { (avg) } \\
\mu \mathrm{g} / \mathrm{kg}\end{array}$ & $\begin{array}{l}\text { Budd } \\
\mu \mathrm{g} / \mathrm{kg}\end{array}$ & $\begin{array}{c}\text { Sullivan } \\
\mu \mathrm{g} / \mathrm{kg}\end{array}$ & $\begin{array}{c}\text { Red Sand } \\
\text { (avg) } \\
\mu \mathrm{g} / \mathrm{kg}\end{array}$ & $\begin{array}{c}\text { Shingobee } \\
\text { (avg) } \\
\mu \mathrm{g} / \mathrm{kg}\end{array}$ & $\begin{array}{l}\text { White } \\
\text { Sand } \\
\mu \mathrm{g} / \mathrm{kg}\end{array}$ & $\begin{array}{c}\text { Stewart } \\
\mu \mathrm{g} / \mathrm{kg}\end{array}$ & $\begin{array}{c}\text { Kabe- } \\
\text { togama } \\
\mu \mathrm{g} / \mathrm{kg}\end{array}$ & $\begin{array}{c}\text { Northern } \\
\text { Light } \\
\mu \mathrm{g} / \mathrm{kg}\end{array}$ & $\begin{array}{c}\text { Elk } \\
\mu \mathrm{g} / \mathrm{kg}\end{array}$ \\
\hline d6-Bisphenol A & 59 & 51 & 54 & 48 & 75 & 61 & 87 & 25 & 54 & 99 & 55 \\
\hline d21-2,6-Di-tert-butyl-4-methylphenol & 19 & 26 & 4 & 5 & 48 & 24 & 95 & 3 & 17 & 53 & 4 \\
\hline 4-normal-Nonylphenol & 114 & 108 & 140 & 97 & 133 & 105 & 144 & 45 & 92 & 148 & 117 \\
\hline 4-normal-Nonylphenolmonoethoxylate & 216 & 73 & 229 & 138 & 163 & 137 & 193 & 66 & 183 & 180 & 168 \\
\hline 4-normal-Nonylphenoldiethoxylate & 106 & 66 & 214 & 90 & 101 & 109 & 122 & 43 & 139 & 174 & 48 \\
\hline
\end{tabular}

Replicates, Blanks, and Matrix Spikes

\begin{tabular}{|c|c|c|c|c|c|c|c|c|c|c|c|c|}
\hline Compound & $\begin{array}{l}\text { Cedar } \\
\mu \mathrm{g} / \mathrm{kg}\end{array}$ & $\begin{array}{c}\text { Cedar } \\
\text { Dup } \\
\mu \mathrm{g} / \mathrm{kg}\end{array}$ & $\begin{array}{l}\text { Cedar } \\
\text { Trip } \\
\mu \mathrm{g} / \mathrm{kg}\end{array}$ & $\begin{array}{l}\text { Red } \\
\text { Sand } \\
\mu \mathrm{g} / \mathrm{kg}\end{array}$ & $\begin{array}{c}\text { Red Sand } \\
\text { Dup } \\
\mu \mathrm{g} / \mathrm{kg}\end{array}$ & $\begin{array}{l}\text { Shin- } \\
\text { gobee } \\
\mu \mathrm{g} / \mathrm{kg}\end{array}$ & $\begin{array}{c}\text { Shingobee } \\
\text { Dup } \\
\mu \mathrm{g} / \mathrm{kg}\end{array}$ & $\begin{array}{l}\text { Sand } \\
\text { Blank } \\
\mu \mathrm{g} / \mathrm{kg}\end{array}$ & $\begin{array}{l}\text { Sand } \\
\text { Blank } \\
\mu \mathrm{g} / \mathrm{kg}\end{array}$ & $\begin{array}{l}\text { Sand } \\
\text { Blank } \\
\mu \mathrm{g} / \mathrm{kg}\end{array}$ & $\begin{array}{c}\text { Red Sand } \\
\text { MS } \\
\text { percent }\end{array}$ & $\begin{array}{c}\text { Red Sand } \\
\text { MS Dup } \\
\text { percent }\end{array}$ \\
\hline Bisphenol A & $<1$ & $<1$ & 6 & 5 & 28 & 14 & 24 & $<1$ & $<1$ & $<1$ & 119 & 95 \\
\hline 2[3]-tert-Butyl-4-methylphenol & $<1$ & $<1$ & $<1$ & $<1$ & $<1$ & $<1$ & $<1$ & $<1$ & $<1$ & $<1$ & 77 & 91 \\
\hline 4-tert-Butylphenol & $<1$ & $<1$ & $<1$ & $<1$ & $<1$ & $<1$ & $<1$ & $<1$ & $<1$ & $<1$ & 178 & 150 \\
\hline Caffeine & $<1$ & 53 & 161 & $<1$ & $<1$ & $<1$ & $<1$ & $<1$ & $<1$ & $<1$ & 0 & 0 \\
\hline 1,2-Dichlorobenzene & $<1$ & $<1$ & $<1$ & $<1$ & $<1$ & $<1$ & $<1$ & $<1$ & $<1$ & $<1$ & 8 & 7 \\
\hline 1,3-Dichlorobenzene & $<1$ & $<1$ & $<1$ & $<1$ & $<1$ & $<1$ & $<1$ & $<1$ & $<1$ & $<1$ & 4 & 3 \\
\hline 1,4-Dichlorobenzene & $<1$ & $<1$ & $<1$ & 1 & $<1$ & 2 & $<1$ & 1 & $<1$ & $<1$ & 5 & 4 \\
\hline 2,6-Di-tert-butyl-1,4-benzoquinone & $<200$ & $<200$ & $<200$ & $<200$ & $<200$ & $<200$ & $<200$ & $<200$ & $<200$ & $<200$ & 28 & 25 \\
\hline 2,6-Di-tert-butyl-4-methylphenol & $<3$ & $<3$ & $<3$ & $<3$ & $<3$ & $<3$ & $<3$ & $<3$ & $<3$ & $<3$ & 107 & 90 \\
\hline 2,6-Di-tert-butylphenol & $<1$ & $<1$ & $<1$ & $<1$ & $<1$ & $<1$ & $<1$ & $<1$ & $<1$ & $<1$ & 89 & 79 \\
\hline$N, N$-Diethyl-meta-toluamide & $<1$ & $<1$ & $<1$ & $<1$ & $<1$ & $<1$ & $<1$ & $<1$ & $<1$ & $<1$ & 18 & 72 \\
\hline 4-Ethylphenol & 10 & $<3$ & 7 & $<3$ & $<3$ & 70 & 122 & $<3$ & $<3$ & $<3$ & 78 & 126 \\
\hline 5-methyl-1H-Benzotriazole & $<1$ & $<1$ & $<1$ & $<1$ & $<1$ & $<1$ & $<1$ & $<1$ & $<1$ & $<1$ & 4 & 10 \\
\hline
\end{tabular}


Table 11. Summary of neutral organic compound data for sediment samples collected from 11 Minnesota lakes during 2008.-Continued

[avg, average; $\mu \mathrm{g} / \mathrm{kg}$, microgram per kilogram; <, less than; MS, matrix spike recovery in percent; Dup, duplicate sample; Trip, triplicate sample]

\begin{tabular}{|c|c|c|c|c|c|c|c|c|c|c|c|c|}
\hline Compound & $\begin{array}{l}\text { Cedar } \\
\mu \mathrm{g} / \mathrm{kg}\end{array}$ & $\begin{array}{c}\text { Cedar } \\
\text { Dup } \\
\mu \mathrm{g} / \mathrm{kg}\end{array}$ & $\begin{array}{c}\text { Cedar } \\
\text { Trip } \\
\mu \mathrm{g} / \mathrm{kg}\end{array}$ & $\begin{array}{l}\text { Red } \\
\text { Sand } \\
\mu \mathrm{g} / \mathrm{kg}\end{array}$ & $\begin{array}{c}\text { Red Sand } \\
\text { Dup } \\
\mu \mathrm{g} / \mathrm{kg}\end{array}$ & $\begin{array}{l}\text { Shin- } \\
\text { gobee } \\
\mu \mathrm{g} / \mathrm{kg}\end{array}$ & $\begin{array}{c}\text { Shingobee } \\
\text { Dup } \\
\mu \mathrm{g} / \mathrm{kg}\end{array}$ & $\begin{array}{l}\text { Sand } \\
\text { Blank } \\
\mu \mathrm{g} / \mathrm{kg}\end{array}$ & $\begin{array}{l}\text { Sand } \\
\text { Blank } \\
\mu \mathrm{g} / \mathrm{kg}\end{array}$ & $\begin{array}{l}\text { Sand } \\
\text { Blank } \\
\mu \mathrm{g} / \mathrm{kg}\end{array}$ & $\begin{array}{c}\text { Red Sand } \\
\text { MS } \\
\text { percent }\end{array}$ & $\begin{array}{l}\text { Red Sand } \\
\text { MS Dup } \\
\text { percent }\end{array}$ \\
\hline 4-Methylphenol & $<3$ & 6 & 18 & 31 & 680 & 108 & 213 & $<3$ & $<3$ & $<3$ & 14 & 122 \\
\hline 4-Nonylphenol & $<100$ & $<100$ & $<100$ & 103 & $<100$ & $<100$ & 349 & $<100$ & $<100$ & $<100$ & 208 & 168 \\
\hline 4-Nonylphenolmonoethoxylate & $<20$ & $<20$ & $<20$ & $<20$ & $<20$ & 30 & $<20$ & $<20$ & $<20$ & $<20$ & 153 & 124 \\
\hline 4-Nonylphenoldiethoxylate & $<1$ & $<1$ & $<1$ & $<1$ & $<1$ & 59 & $<1$ & $<1$ & $<1$ & $<1$ & 119 & 114 \\
\hline 4-Nonylphenoltriethoxylate & $<1$ & $<1$ & $<1$ & $<1$ & $<1$ & $<1$ & $<1$ & $<1$ & $<1$ & $<1$ & 130 & 115 \\
\hline 4-Nonylphenoltetraethoxylate & $<1$ & $<1$ & $<1$ & $<1$ & $<1$ & $<1$ & $<1$ & $<1$ & $<1$ & $<1$ & 33 & 32 \\
\hline 4-normal-Octylphenol & $<1$ & $<1$ & $<1$ & $<1$ & $<1$ & $<1$ & $<1$ & $<1$ & $<1$ & $<1$ & 241 & 193 \\
\hline 4-tert-Octylphenol & $<3$ & $<3$ & 4 & $<3$ & $<3$ & 22 & $<3$ & $<3$ & $<3$ & $<3$ & 216 & 183 \\
\hline 4-tert-Octylphenolmonoethoxylate & $<1$ & $<1$ & $<1$ & $<1$ & 1 & $<1$ & 6 & $<1$ & $<1$ & $<1$ & 207 & 174 \\
\hline 4-tert-Octylphenoldiethoxylate & $<1$ & $<1$ & $<1$ & 21 & $<1$ & $<1$ & $<1$ & $<1$ & $<1$ & $<1$ & 105 & 95 \\
\hline 4-tert-Octylphenoltriethoxylate & $<1$ & $<1$ & $<1$ & $<1$ & $<1$ & $<1$ & $<1$ & $<1$ & $<1$ & $<1$ & 108 & 100 \\
\hline 4-tert-Octylphenoltetraethoxylate & $<1$ & $<1$ & $<1$ & $<1$ & $<1$ & $<1$ & $<1$ & $<1$ & $<1$ & $<1$ & 173 & 151 \\
\hline 4-tert-Octylphenolpentathoxylate & $<1$ & $<1$ & $<1$ & $<1$ & $<1$ & $<1$ & $<1$ & $<1$ & $<1$ & $<1$ & 0 & 0 \\
\hline 4-tert-Pentylphenol & $<1$ & $<1$ & $<1$ & $<1$ & $<1$ & $<1$ & $<1$ & $<1$ & $<1$ & $<1$ & 190 & 150 \\
\hline 4-Propylphenol & $<1$ & $<1$ & $<1$ & $<1$ & $<1$ & $<1$ & $<1$ & $<1$ & $<1$ & $<1$ & 176 & 152 \\
\hline Triclosan & $<1$ & $<1$ & 44 & $<1$ & $<1$ & $<1$ & $<1$ & $<1$ & $<1$ & $<1$ & 184 & 156 \\
\hline \multicolumn{13}{|l|}{ Surrogate Recovery (percent) } \\
\hline d6-Bisphenol A & 44 & 44 & 65 & 91 & 59 & 58 & 63 & 68 & 46 & 58 & 110 & 65 \\
\hline d21-2,6-Di-tert-butyl-4-methylphenol & 29 & 22 & 29 & 39 & 56 & 29 & 19 & 64 & 33 & 60 & 86 & 59 \\
\hline 4-normal-Nonylphenol & 85 & 121 & 117 & 136 & 129 & 99 & 110 & 105 & 117 & 142 & 199 & 119 \\
\hline 4-normal-Nonylphenolmonoethoxylate & 0 & 111 & 106 & 173 & 152 & 117 & 156 & 70 & 120 & 46 & 343 & 220 \\
\hline 4-normal-Nonylphenoldiethoxylate & 49 & 80 & 68 & 123 & 80 & 91 & 127 & 52 & 72 & 32 & 113 & 77 \\
\hline
\end{tabular}


Table 12. Summary of steroid and steroidal hormone compound data for sediment samples collected from 11 Minnesota lakes during 2008.

[avg, average; $\mu \mathrm{g} / \mathrm{kg}$, microgram per kilogram; <, less than; Dup, duplicate sample; Trip, triplicate sample; MS, matrix spike recovery in percent]

\begin{tabular}{|c|c|c|c|c|c|c|c|c|c|c|c|}
\hline Compound & $\begin{array}{c}\text { Owasso } \\
\mu \mathrm{g} / \mathrm{kg}\end{array}$ & $\begin{array}{c}\text { Cedar (avg) } \\
\mu \mathrm{\mu g} / \mathrm{kg}\end{array}$ & $\begin{array}{l}\text { Budd } \\
\mu g / \mathbf{k g}\end{array}$ & $\begin{array}{c}\text { Sullivan } \\
\mu \mathrm{g} / \mathbf{k g}\end{array}$ & $\begin{array}{c}\text { Red Sand } \\
\text { (avg) } \\
\mu \mathrm{g} / \mathrm{kg}\end{array}$ & $\begin{array}{c}\text { Shingobee } \\
\text { (avg) } \\
\mu \mathrm{g} / \mathbf{k g}\end{array}$ & $\begin{array}{c}\text { White Sand } \\
\mu \mathrm{g} / \mathrm{kg}\end{array}$ & $\begin{array}{c}\text { Stewart } \\
\mu \mathrm{g} / \mathrm{kg}\end{array}$ & $\begin{array}{c}\text { Kabetogama } \\
\mu \mathrm{g} / \mathrm{kg}\end{array}$ & $\begin{array}{c}\text { Northern } \\
\text { Light } \\
\mu \mathrm{g} / \mathrm{kg}\end{array}$ & $\begin{array}{c}\text { Elk } \\
\mu \mathrm{g} / \mathrm{kg}\end{array}$ \\
\hline 4-Androstene-3,17-dione & 2.3 & $<0.05$ & 0.22 & 0.39 & 0.55 & 0.94 & 0.59 & 0.33 & $<0.05$ & 0.61 & 0.47 \\
\hline cis-Androsterone & $<.05$ & $<.05$ & $<.05$ & $<.05$ & $<.05$ & $<.05$ & $<.05$ & $<.05$ & $<.05$ & $<.05$ & $<.05$ \\
\hline Cholesterol & 4,200 & 330 & 530 & 1,400 & 1,200 & 1,600 & 770 & 1,200 & 3,600 & 320 & 1,800 \\
\hline Coprostanol & 790 & 47 & 32 & 360 & 320 & 340 & 35 & 130 & 380 & 36 & 230 \\
\hline Diethylstilbesterol & $<.05$ & $<.05$ & $<.05$ & $<.05$ & $<.05$ & $<.05$ & $<.05$ & $<.05$ & 3.8 & .36 & $<.05$ \\
\hline Equilenin & $<.05$ & $<.05$ & $<.05$ & $<.05$ & $<.05$ & $<.05$ & $<.05$ & $<.05$ & $<.05$ & $<.05$ & $<.05$ \\
\hline Equilin & $<5$ & $<5$ & $<5$ & $<5$ & $<5$ & $<5$ & $<5$ & $<5$ & $<5$ & $<5$ & $<5$ \\
\hline $17 \alpha$-Estradiol & $<.05$ & $<.05$ & .08 & .06 & .10 & .17 & .46 & .08 & $<.05$ & $<.05$ & $<.05$ \\
\hline $17 \beta$-Estradiol & 1.3 & $<.05$ & .05 & .15 & .35 & 1.3 & .58 & .12 & .85 & .08 & .23 \\
\hline Estriol & $<.05$ & $<.05$ & $<.05$ & $<.05$ & $<.05$ & $<.05$ & $<.05$ & $<.05$ & $<.05$ & $<.05$ & $<.05$ \\
\hline Estrone & 2.9 & $<.05$ & .27 & .82 & .69 & 2.4 & .99 & .59 & 5.6 & .32 & 3.1 \\
\hline $17 \alpha$-Ethinylestradiol & $<.05$ & $<.05$ & $<.05$ & $<.05$ & $<.05$ & $<.05$ & $<.05$ & $<.05$ & $<.05$ & $<.05$ & $<.05$ \\
\hline Mestranol & $<.05$ & $<.05$ & $<.05$ & $<.05$ & $<.05$ & $<.05$ & $<.05$ & $<.05$ & $<.05$ & $<.05$ & $<.05$ \\
\hline Norethindrone & $<.05$ & $<.05$ & $<.05$ & $<.05$ & $<.05$ & $<.05$ & $<.05$ & $<.05$ & $<.05$ & $<.05$ & $<.05$ \\
\hline Progesterone & $<.05$ & $<.05$ & $<.05$ & $<.05$ & $<.05$ & $<.05$ & $<.05$ & $<.05$ & $<.05$ & $<.05$ & $<.05$ \\
\hline Testosterone & 1.4 & $<.05$ & $<.05$ & $<.05$ & .26 & $<.05$ & .23 & $<.05$ & 2.7 & $<.05$ & $<.05$ \\
\hline dihydro-Testosterone & $<.05$ & $<.05$ & $<.05$ & $<.05$ & $<.05$ & $<.05$ & $<.05$ & $<.05$ & $<.05$ & $<.05$ & $<.05$ \\
\hline epi-Testosterone & $<.05$ & $<.05$ & $<.05$ & $<.05$ & .14 & $<.05$ & $<.05$ & $<.05$ & .89 & $<.05$ & $<.05$ \\
\hline 11-keto-Testosterone & $<.05$ & $<.05$ & $<.05$ & $<.05$ & $<.05$ & $<.05$ & $<.05$ & $<.05$ & $<.05$ & $<.05$ & $<.05$ \\
\hline \multicolumn{12}{|c|}{ Surrogate Recovery (percent) } \\
\hline d7-Androstenedione & 19 & 0 & 33 & 13 & 15 & 28 & 26 & 10 & 51 & 37 & 11 \\
\hline d7-Cholesterol & 7 & 1 & 9 & 11 & 20 & 12 & 13 & 4 & 39 & 16 & 8 \\
\hline d8-Diethylstilbesterol & 25 & 0 & 25 & 64 & 54 & 43 & 50 & 22 & 24 & 26 & 64 \\
\hline d4-Dihydrotestosterone & 24 & 2 & 53 & 16 & 26 & 43 & 46 & 15 & 56 & 58 & 27 \\
\hline d4-Ethinylestradiol & 41 & 3 & 40 & 37 & 62 & 63 & 68 & 33 & 92 & 64 & 59 \\
\hline d4-Estradiol & 25 & 2 & 34 & 47 & 53 & 54 & 58 & 22 & 33 & 20 & 38 \\
\hline d4-Mestranol & 47 & 3 & 75 & 39 & 63 & 83 & 2 & 38 & 94 & 71 & 80 \\
\hline d6-Norethindrone & 11 & 0 & 21 & 8 & 17 & 16 & 18 & 5 & 27 & 23 & 8 \\
\hline d5-Testosterone & 23 & 1 & 33 & 15 & 21 & 32 & 32 & 10 & 51 & 38 & 14 \\
\hline
\end{tabular}


Replicates, Blanks, and Matrix Spikes

Table 12. Summary of steroid and steroidal hormone compound data for sediment samples collected from 11 Minnesota lakes during 2008.-Continued [avg, average; $\mu \mathrm{g} / \mathrm{kg}$, microgram per kilogram; <, less than; Dup, duplicate sample; Trip, triplicate sample; MS, matrix spike recovery in percent]

\begin{tabular}{|c|c|c|c|c|c|c|c|c|c|c|c|c|}
\hline Compound & $\begin{array}{l}\text { Cedar } \\
\mu \mathrm{g} / \mathrm{kg}\end{array}$ & $\begin{array}{c}\text { Cedar Dup } \\
\mu \mathrm{\mu g} / \mathrm{kg}\end{array}$ & $\begin{array}{c}\text { Cedar Trip } \\
\mu \mathrm{g} / \mathrm{kg}\end{array}$ & $\begin{array}{c}\text { Red Sand } \\
\mu \mathrm{g} / \mathrm{kg}\end{array}$ & $\begin{array}{c}\text { Red Sand } \\
\text { Dup } \\
\mu \mathrm{g} / \mathrm{kg}\end{array}$ & $\begin{array}{c}\text { Shingobee } \\
\mu \mathrm{g} / \mathrm{kg}\end{array}$ & $\begin{array}{c}\text { Shingobee } \\
\text { Dup } \\
\mu \mathrm{g} / \mathrm{kg}\end{array}$ & $\begin{array}{l}\text { Sand } \\
\text { Blank } \\
\mu \mathrm{g} / \mathrm{kg}\end{array}$ & $\begin{array}{l}\text { Sand } \\
\text { Blank } \\
\mu \mathrm{g} / \mathrm{kg}\end{array}$ & $\begin{array}{l}\text { Sand } \\
\text { Blank } \\
\mu \mathrm{g} / \mathrm{kg}\end{array}$ & $\begin{array}{c}\text { Red Sand } \\
\text { MS } \\
\mu \mathrm{g} / \mathrm{kg}\end{array}$ & $\begin{array}{c}\text { Red Sand } \\
\text { MS Dup } \\
\mu g / k g\end{array}$ \\
\hline 4-Androstene-3,17-dione & $<.05$ & $<.05$ & $<.05$ & $<.05$ & .55 & .97 & .92 & $<.05$ & .11 & .06 & 25 & 94 \\
\hline cis-Androsterone & $<.05$ & $<.05$ & $<.05$ & $<.05$ & $<.05$ & $<.05$ & $<.05$ & $<.05$ & $<.05$ & $<.05$ & 86 & 100 \\
\hline Cholesterol & 420 & 320 & 260 & 870 & 1400 & 910 & 2400 & 41 & 72 & 12 & 52 & 90 \\
\hline Coprostanol & 50 & 51 & 41 & 270 & 370 & 220 & 460 & $<5$ & $<5$ & $<5$ & 30 & 44 \\
\hline Diethylstilbesterol & $<.05$ & $<.05$ & $<.05$ & $<.05$ & $<.05$ & $<.05$ & $<.05$ & $<.05$ & $<.05$ & $<.05$ & 71 & 120 \\
\hline Equilenin & $<.05$ & $<.05$ & $<.05$ & $<.05$ & $<.05$ & $<.05$ & $<.05$ & $<.05$ & $<.05$ & $<.05$ & 87 & 92 \\
\hline Equilin & $<5$ & $<5$ & $<5$ & $<5$ & $<5$ & $<5$ & $<5$ & $<5$ & $<5$ & $<5$ & 17 & 19 \\
\hline $17 \alpha$-Estradiol & $<.05$ & $<.05$ & $<.05$ & .07 & .13 & .16 & .17 & $<.05$ & $<.05$ & $<.05$ & 57 & 68 \\
\hline $17 \beta$-Estradiol & $<.05$ & $<.05$ & $<.05$ & .20 & .49 & 1.00 & 1.58 & $<.05$ & $<.05$ & $<.05$ & 53 & 66 \\
\hline Estriol & $<.05$ & $<.05$ & $<.05$ & $<.05$ & $<.05$ & $<.05$ & $<.05$ & $<.05$ & $<.05$ & $<.05$ & 0 & 77 \\
\hline Estrone & $<.05$ & $<.05$ & $<.05$ & .30 & 1.08 & 1.98 & 2.78 & $<.05$ & $<.05$ & $<.05$ & 43 & 54 \\
\hline $17 \alpha$-Ethinylestradiol & $<.05$ & $<.05$ & $<.05$ & $<.05$ & $<.05$ & $<.05$ & $<.05$ & $<.05$ & $<.05$ & $<.05$ & 77 & 77 \\
\hline Mestranol & $<.05$ & $<.05$ & $<.05$ & $<.05$ & $<.05$ & $<.05$ & $<.05$ & $<.05$ & $<.05$ & $<.05$ & 60 & 78 \\
\hline Norethindrone & $<.05$ & $<.05$ & $<.05$ & $<.05$ & $<.05$ & $<.05$ & $<.05$ & $<.05$ & $<.05$ & $<.05$ & 0 & 139 \\
\hline Progesterone & $<.05$ & $<.05$ & $<.05$ & $<.05$ & $<.05$ & $<.05$ & $<.05$ & $<.05$ & $<.05$ & $<.05$ & 0 & 0 \\
\hline Testosterone & $<.05$ & $<.05$ & $<.05$ & $<.05$ & .26 & $<.05$ & $<.05$ & $<.05$ & $<.05$ & $<.05$ & 118 & 104 \\
\hline dihydro-Testosterone & $<.05$ & $<.05$ & $<.05$ & $<.05$ & $<.05$ & $<.05$ & $<.05$ & $<.05$ & $<.05$ & $<.05$ & & \\
\hline epi-Testosterone & $<.05$ & $<.05$ & $<.05$ & $<.05$ & .14 & $<.05$ & $<.05$ & $<.05$ & $<.05$ & $<.05$ & 126 & 106 \\
\hline 11-keto-Testosterone & $<.05$ & $<.05$ & $<.05$ & $<.05$ & $<.05$ & $<.05$ & $<.05$ & $<.05$ & $<.05$ & $<.05$ & 0 & 0 \\
\hline \multicolumn{13}{|c|}{ Surrogate Recovery (percent) } \\
\hline d7-Androstenedione & 1 & 0 & 0 & 6 & 25 & 32 & 24 & 19 & 12 & 18 & 5 & 20 \\
\hline d7-Cholesterol & 1 & 0 & 1 & 29 & 12 & 12 & 11 & 10 & 14 & 33 & 45 & 27 \\
\hline d8-Diethylstilbesterol & 0 & 0 & 0 & 56 & 52 & 39 & 46 & 32 & 0 & 65 & 77 & 62 \\
\hline d4-Dihydrotestosterone & 2 & 0 & 2 & 14 & 38 & 52 & 34 & 63 & 50 & 69 & 35 & 46 \\
\hline d4-Ethinyl estradiol & 3 & 1 & 4 & 65 & 60 & 64 & 62 & 54 & 20 & 67 & 95 & 83 \\
\hline d4-Estradiol & 3 & 1 & 3 & 55 & 52 & 49 & 60 & 60 & 29 & 126 & 84 & 53 \\
\hline d4-Mestranol & 4 & 1 & 5 & 57 & 69 & 95 & 72 & 64 & 51 & 72 & 88 & 74 \\
\hline d6-Norethindrone & 0 & 0 & 0 & 2 & 32 & 19 & 13 & 15 & 10 & 18 & 0 & 7 \\
\hline d5-Testosterone & 1 & 0 & 1 & 6 & 35 & 36 & 28 & 31 & 22 & 33 & 6 & 34 \\
\hline
\end{tabular}


Table 13. Summary of neutral organic compound data for polar organic compound integrative samplers (POCIS) deployed in 11 Minnesota lakes during 2008.

[ $\mu \mathrm{g} /$ POCIS, microgram per POCIS; $<$, less than]

\begin{tabular}{|c|c|c|c|c|c|c|c|c|c|c|c|c|c|c|}
\hline Compound & $\begin{array}{c}\text { Owasso } \\
\text { pg/ } \\
\text { POCIS }\end{array}$ & $\begin{array}{c}\text { Cedar } \\
\mu \mathrm{gg} / \\
\text { POCIS }\end{array}$ & $\begin{array}{c}\text { Budd } \\
\mu \mathrm{g} / \\
\text { POCIS }\end{array}$ & $\begin{array}{c}\text { Sullivan } \\
\mu \mathrm{\mu g} / \\
\text { POCIS }\end{array}$ & $\begin{array}{c}\text { Sullivan } \\
\text { Day } 7 \\
\mu g / \\
\text { POCIS }\end{array}$ & $\begin{array}{c}\text { Sullivan } \\
\text { Day } 14 \\
\mu \mathrm{pg} / \\
\text { POCIS }\end{array}$ & $\begin{array}{c}\text { Sullivan } \\
\text { Day 21 } \\
\mu \mathrm{g} / \\
\text { POCIS }\end{array}$ & $\begin{array}{l}\text { Red } \\
\text { Sand } \\
\mu \mathrm{g} / \\
\text { POCIS }\end{array}$ & $\begin{array}{c}\text { Shingobee } \\
\text { pg/ } \\
\text { POCIS }\end{array}$ & $\begin{array}{l}\text { White } \\
\text { Sand } \\
\mu g / \\
\text { POCIS }\end{array}$ & $\begin{array}{c}\text { Stewart } \\
\mu \mathrm{g} / \\
\text { POCIS }\end{array}$ & $\begin{array}{c}\text { Kabetogama } \\
\text { pg/ } \\
\text { POCIS }\end{array}$ & $\begin{array}{c}\text { Northern } \\
\text { Light } \\
\mu \mathrm{g} / \\
\text { POCIS }\end{array}$ & $\begin{array}{c}\text { Elk } \\
\text { pg/ } \\
\text { POCIS }\end{array}$ \\
\hline Bisphenol A & $<0.01$ & $<0.01$ & $<0.01$ & 0.01 & $<0.01$ & $<0.01$ & $<0.01$ & $<0.01$ & $<0.01$ & $<0.01$ & $<0.01$ & $<0.01$ & $<0.01$ & $<0.01$ \\
\hline 2[3]-tert-Butyl-4-methylphenol & $<.01$ & $<.01$ & $<.01$ & $<.01$ & $<.01$ & $<.01$ & $<.01$ & $<.01$ & $<.01$ & $<.01$ & $<.01$ & $<.01$ & $<.01$ & $<.01$ \\
\hline 4-tert-Butylphenol & $<.01$ & $<.01$ & $<.01$ & $<.01$ & $<.01$ & $<.01$ & $<.01$ & $<.01$ & $<.01$ & $<.01$ & $<.01$ & $<.01$ & $<.01$ & $<.01$ \\
\hline Caffeine & .02 & .11 & $<.01$ & $<.01$ & $<.01$ & $<.01$ & $<.01$ & $<.01$ & $<.01$ & $<.01$ & $<.01$ & $<.01$ & $<.01$ & $<.01$ \\
\hline 1,2-Dichlorobenzene & $<.01$ & $<.01$ & $<.01$ & $<.01$ & $<.01$ & $<.01$ & $<.01$ & $<.01$ & $<.01$ & $<.01$ & $<.01$ & $<.01$ & $<.01$ & $<.01$ \\
\hline 1,3-Dichlorobenzene & $<.01$ & $<.01$ & $<.01$ & $<.01$ & $<.01$ & $<.01$ & $<.01$ & $<.01$ & $<.01$ & $<.01$ & $<.01$ & $<.01$ & $<.01$ & $<.01$ \\
\hline 1,4-Dichlorobenzene & $<.01$ & $<.01$ & $<.01$ & $<.01$ & $<.01$ & $<.01$ & $<.01$ & $<.01$ & $<.01$ & $<.01$ & $<.01$ & $<.01$ & $<.01$ & $<.01$ \\
\hline 2,6-Di-tert-butyl-1,4-benzoquinone & $<.01$ & $<.01$ & $<.01$ & .05 & .07 & .08 & .05 & .04 & .29 & .02 & .21 & .18 & .08 & .06 \\
\hline 2,6-Di-tert-butyl-4-methylphenol & .35 & $<.01$ & $<.01$ & .22 & .22 & .24 & $<.01$ & .17 & .02 & .06 & .01 & .16 & .18 & .18 \\
\hline 2,6-Di-tert-butylphenol & $<.01$ & $<.01$ & $<.01$ & $<.01$ & $<.01$ & $<.01$ & $<.01$ & $<.01$ & $<.01$ & $<.01$ & $<.01$ & $<.01$ & $<.01$ & $<.01$ \\
\hline$N, N$-Diethyl-meta-toluamide & .12 & .09 & $<.01$ & .08 & .02 & .02 & .04 & .03 & .01 & .09 & .06 & .02 & .04 & .03 \\
\hline 4-Ethylphenol & .02 & .04 & $<.01$ & $<.01$ & $<.01$ & $<.01$ & .01 & $<.01$ & .01 & $<.01$ & .01 & .01 & $<.01$ & .03 \\
\hline 5-methyl-1H-Benzotriazole & $<.01$ & .06 & $<.01$ & $<.01$ & $<.01$ & $<.01$ & $<.01$ & $<.01$ & $<.01$ & $<.01$ & $<.01$ & $<.01$ & $<.01$ & $<.01$ \\
\hline 4-Methylphenol & .06 & .03 & .02 & .06 & .01 & .01 & .02 & .04 & .06 & .01 & .06 & .26 & .04 & .03 \\
\hline 4-Nonylphenol & .09 & .06 & .03 & .09 & .05 & .04 & .06 & .12 & .06 & .08 & .09 & .05 & .06 & .05 \\
\hline 4-Nonylphenolmonoethoxylate & $<.01$ & $<.01$ & $<.01$ & .04 & $<.01$ & $<.01$ & .03 & .03 & $<.01$ & .02 & $<.01$ & $<.01$ & $<.01$ & $<.01$ \\
\hline 4-Nonylphenoldiethoxylate & $<.01$ & $<.01$ & $<.01$ & $<.01$ & $<.01$ & $<.01$ & $<.01$ & .12 & $<.01$ & $<.01$ & $<.01$ & $<.01$ & $<.01$ & $<.01$ \\
\hline 4-Nonylphenoltriethoxylate & $<.01$ & $<.01$ & $<.01$ & .06 & $<.01$ & $<.01$ & $<.01$ & $<.01$ & $<.01$ & .09 & $<.01$ & $<.01$ & $<.01$ & $<.01$ \\
\hline 4-Nonylphenoltetraethoxylate & $<.01$ & $<.01$ & $<.01$ & $<.01$ & $<.01$ & $<.01$ & $<.01$ & $<.01$ & $<.01$ & $<.01$ & $<.01$ & $<.01$ & $<.01$ & $<.01$ \\
\hline 4-normal-Octylphenol & $<.01$ & $<.01$ & $<.01$ & $<.01$ & $<.01$ & $<.01$ & $<.01$ & $<.01$ & $<.01$ & $<.01$ & $<.01$ & $<.01$ & $<.01$ & $<.01$ \\
\hline 4-tert-Octylphenol & $<.01$ & $<.01$ & $<.01$ & $<.01$ & $<.01$ & $<.01$ & $<.01$ & $<.01$ & $<.01$ & $<.01$ & $<.01$ & $<.01$ & $<.01$ & $<.01$ \\
\hline 4-tert-Octylphenolmonoethoxylate & $<.01$ & $<.01$ & $<.01$ & $<.01$ & $<.01$ & $<.01$ & $<.01$ & $<.01$ & $<.01$ & $<.01$ & $<.01$ & $<.01$ & $<.01$ & $<.01$ \\
\hline 4-tert-Octylphenoldiethoxylate & $<.01$ & $<.01$ & $<.01$ & $<.01$ & .02 & $<.01$ & $<.01$ & $<.01$ & $<.01$ & $<.01$ & $<.01$ & $<.01$ & $<.01$ & $<.01$ \\
\hline 4-tert-Octylphenoltriethoxylate & $<.01$ & $<.01$ & $<.01$ & $<.01$ & $<.01$ & $<.01$ & $<.01$ & $<.01$ & $<.01$ & $<.01$ & $<.01$ & $<.01$ & $<.01$ & $<.01$ \\
\hline 4-tert-Octylphenoltetraethoxylate & $<.01$ & $<.01$ & $<.01$ & $<.01$ & $<.01$ & $<.01$ & $<.01$ & $<.01$ & $<.01$ & $<.01$ & $<.01$ & $<.01$ & $<.01$ & $<.01$ \\
\hline 4-tert-Octylphenolpentaethoxylate & $<.01$ & $<.01$ & $<.01$ & $<.01$ & $<.01$ & $<.01$ & $<.01$ & $<.01$ & $<.01$ & $<.01$ & $<.01$ & $<.01$ & $<.01$ & $<.01$ \\
\hline 4-tert-Pentylphenol & $<.01$ & $<.01$ & $<.01$ & $<.01$ & $<.01$ & $<.01$ & $<.01$ & $<.01$ & $<.01$ & $<.01$ & $<.01$ & $<.01$ & $<.01$ & $<.01$ \\
\hline 4-Propylphenol & $<.01$ & $<.01$ & $<.01$ & $<.01$ & $<.01$ & $<.01$ & $<.01$ & $<.01$ & $<.01$ & $<.01$ & $<.01$ & $<.01$ & $<.01$ & $<.01$ \\
\hline Triclosan & $<.01$ & $<.01$ & $<.01$ & $<.01$ & $<.01$ & $<.01$ & $<.01$ & $<.01$ & $<.01$ & $<.01$ & $<.01$ & $<.01$ & $<.01$ & $<.01$ \\
\hline \multicolumn{15}{|l|}{ Surrogate Recovery (percent) } \\
\hline d6-Bisphenol A & 22 & 20 & 9 & 147 & 167 & 92 & 107 & 123 & 161 & 131 & 144 & 99 & 125 & 123 \\
\hline d21-2,6-Di-tert-butyl-4-methylphenol & 13 & 3 & 6 & 23 & 36 & 36 & 8 & 8 & 73 & 3 & 40 & 48 & 17 & 70 \\
\hline 4-normal-Nonylphenol & 23 & 17 & 8 & 144 & 146 & 50 & 106 & 116 & 143 & 116 & 133 & 101 & 115 & 99 \\
\hline 4-normal-Nonylphenolmonoethoxylate & 18 & 13 & 6 & 197 & 187 & 53 & 130 & 155 & 189 & 158 & 170 & 131 & 144 & 128 \\
\hline 4-normal-Nonylphenoldiethoxylate & 96 & 0 & 24 & 685 & 1250 & 261 & 863 & 95 & 1276 & 74 & 92 & 857 & 136 & 90 \\
\hline
\end{tabular}




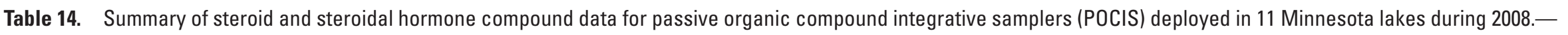
Continued

[avg, average; $\mu \mathrm{g} /$ POCIS, microgram per POCIS; <, less than; Dup, duplicate sample; Trip, triplicate sample; --, not quantified]

\begin{tabular}{|c|c|c|c|c|c|c|c|c|c|c|c|c|c|c|}
\hline Compound & $\begin{array}{c}\text { Owasso } \\
\text { (avg) } \\
\mu g / \\
\text { POCIS }\end{array}$ & $\begin{array}{c}\text { Cedar } \\
\text { (avg) } \\
\mu g / \\
\text { POCIS }\end{array}$ & $\begin{array}{c}\text { Budd } \\
\text { (avg) } \\
\mu g / \\
\text { POCIS }\end{array}$ & $\begin{array}{c}\text { Sullivan } \\
\text { pg/ } \\
\text { POCIS }\end{array}$ & $\begin{array}{c}\text { Sullivan } \\
\text { Day } 7 \\
\mu \mathrm{g} / \\
\text { POCIS }\end{array}$ & $\begin{array}{c}\text { Sullivan } \\
\text { Day } 14 \\
\mu \mathrm{g} / \\
\text { POCIS }\end{array}$ & $\begin{array}{c}\text { Sullivan } \\
\text { Day } 21 \\
\mu \mathrm{g} / \\
\text { POCIS }\end{array}$ & $\begin{array}{c}\text { Red Sand } \\
\mu \mathrm{g} / \\
\text { POCIS }\end{array}$ & $\begin{array}{c}\text { Shingobee } \\
\mu \mathrm{gg} / \\
\text { POCIS }\end{array}$ & $\begin{array}{l}\text { White } \\
\text { Sand } \\
\mu g / \\
\text { POCIS }\end{array}$ & $\begin{array}{c}\text { Stewart } \\
\mu g / \\
\text { POCIS }\end{array}$ & $\begin{array}{c}\text { Kabetogama } \\
\text { pg/ } \\
\text { POCIS }\end{array}$ & $\begin{array}{c}\text { Northern } \\
\text { Light } \\
\mu \mathrm{\mu g} / \\
\text { POCIS }\end{array}$ & $\begin{array}{c}\text { Elk } \\
\text { Hg/ } \\
\text { POCIS }\end{array}$ \\
\hline POCIS extracted & 1 & 1 & 1 & 3 & 3 & 3 & 3 & 3 & 3 & 3 & 3 & 3 & 3 & 3 \\
\hline 4-Androstene-3,17-dione & $<.0001$ & $<.0001$ & .0023 & .0005 & $<.0001$ & $<.0001$ & $<.0001$ & .0007 & $<.0001$ & .0009 & .0007 & $<.0001$ & $<.0001$ & .0008 \\
\hline cis-Androsterone & $<.0001$ & $<.0001$ & $<.0001$ & $<.0001$ & $<.0001$ & $<.0001$ & $<.0001$ & $<.0001$ & $<.0001$ & $<.0001$ & $<.0001$ & $<.0001$ & $<.0001$ & $<.0001$ \\
\hline Cholesterol & $<.5$ & $<.5$ & 4.6 & .5 & $<.5$ & $<.5$ & $<.5$ & .5 & $<.5$ & $<.5$ & $<.5$ & $<.5$ & $<.5$ & $<.5$ \\
\hline Coprostanol & $<.5$ & $<.5$ & $<.5$ & $<.5$ & $<.5$ & $<.5$ & $<.5$ & $<.5$ & $<.5$ & $<.5$ & $<.5$ & $<.5$ & $<.5$ & $<.5$ \\
\hline Diethylstilbesterol & $<.0001$ & $<.0001$ & $<.0001$ & $<.0001$ & $<.0001$ & $<.0001$ & $<.0001$ & $<.0001$ & $<.0001$ & $<.0001$ & $<.0001$ & $<.0001$ & $<.0001$ & $<.0001$ \\
\hline Equilenin & $<.0001$ & $<.0001$ & $<.0001$ & $<.0001$ & $<.0001$ & $<.0001$ & $<.0001$ & $<.0001$ & $<.0001$ & $<.0001$ & $<.0001$ & $<.0001$ & $<.0001$ & $<.0001$ \\
\hline Equilin & $<.5$ & $<.5$ & $<.5$ & $<.5$ & $<.5$ & $<.5$ & $<.5$ & $<.5$ & $<.5$ & $<.5$ & $<.5$ & $<.5$ & $<.5$ & $<.5$ \\
\hline $17 \alpha$-Estradiol & $<.0001$ & $<.0001$ & $<.0001$ & .0001 & $<.0001$ & $<.0001$ & $<.0001$ & $<.0001$ & $<.0001$ & $<.0001$ & $<.0001$ & $<.0001$ & $<.0001$ & .0002 \\
\hline 17ß-Estradiol & .0005 & .0006 & .0003 & .0003 & $<.0001$ & .0001 & $<.0001$ & $<.0001$ & .0001 & .0001 & .0002 & .0001 & $<.0001$ & .0003 \\
\hline Estriol & $<.0001$ & $<.0001$ & $<.0001$ & $<.0001$ & $<.0001$ & $<.0001$ & $<.0001$ & $<.0001$ & $<.0001$ & $<.0001$ & $<.0001$ & $<.0001$ & $<.0001$ & $<.0001$ \\
\hline Estrone & .0012 & .0012 & .0010 & .0013 & $<.0001$ & $<.0001$ & .0004 & .0012 & .0008 & .0011 & .0022 & $<.0001$ & $<.0001$ & .0010 \\
\hline 17 $\alpha$-Ethinylestradiol & $<.0001$ & $<.0001$ & $<.0001$ & $<.0001$ & $<.0001$ & $<.0001$ & $<.0001$ & $<.0001$ & $<.0001$ & $<.0001$ & $<.0001$ & $<.0001$ & $<.0001$ & $<.0001$ \\
\hline Mestranol & $<.0001$ & $<.0001$ & $<.0001$ & $<.0001$ & $<.0001$ & $<.0001$ & $<.0001$ & $<.0001$ & $<.0001$ & $<.0001$ & $<.0001$ & $<.0001$ & $<.0001$ & $<.0001$ \\
\hline Norethindrone & $<.0001$ & $<.0001$ & $<.0001$ & $<.0001$ & $<.0001$ & $<.0001$ & $<.0001$ & $<.0001$ & $<.0001$ & $<.0001$ & $<.0001$ & $<.0001$ & $<.0001$ & $<.0001$ \\
\hline Progesterone & $<.0001$ & $<.0001$ & $<.0001$ & $<.0001$ & $<.0001$ & $<.0001$ & $<.0001$ & $<.0001$ & $<.0001$ & $<.0001$ & $<.0001$ & $<.0001$ & $<.0001$ & $<.0001$ \\
\hline Testosterone & .0004 & $<.0001$ & .0004 & $<.0001$ & $<.0001$ & $<.0001$ & $<.0001$ & $<.0001$ & $<.0001$ & $<.0001$ & $<.0001$ & $<.0001$ & .0002 & $<.0001$ \\
\hline dihydro-Testosterone & $<.0001$ & $<.0001$ & $<.0001$ & $<.0001$ & $<.0001$ & $<.0001$ & $<.0001$ & $<.0001$ & $<.0001$ & $<.0001$ & $<.0001$ & $<.0001$ & $<.0001$ & $<.0001$ \\
\hline epi-Testosterone & $<.0001$ & $<.0001$ & $<.0001$ & $<.0001$ & $<.0001$ & $<.0001$ & $<.0001$ & $<.0001$ & $<.0001$ & $<.0001$ & $<.0001$ & $<.0001$ & $<.0001$ & .0002 \\
\hline 11-keto-Testosterone & $<.0001$ & $<.0001$ & .0017 & $<.0001$ & $<.0001$ & $<.0001$ & $<.0001$ & $<.0001$ & $<.0001$ & $<.0001$ & $<.0001$ & $<.0001$ & $<.0001$ & $<.0001$ \\
\hline \multicolumn{15}{|c|}{ Surrogate Recovery (percent) } \\
\hline d7-Androstenedione & 136 & 132 & 143 & 149 & 109 & 75 & 81 & 93 & 91 & 54 & 107 & 79 & 87 & 80 \\
\hline d7-Cholesterol & 59 & 63 & 83 & 45 & 81 & 23 & 35 & 68 & 28 & 23 & 65 & 30 & 43 & 20 \\
\hline d8-Diethylstilbestrol & 94 & 84 & 91 & 101 & 60 & 37 & 38 & 55 & 68 & 55 & 69 & 45 & 65 & 55 \\
\hline d4-Ethinyl estradiol & 109 & 106 & 124 & 143 & 100 & 65 & 65 & 88 & 68 & 59 & 98 & 57 & 74 & 56 \\
\hline d4-Estradiol & 95 & 88 & 104 & 1 & 139 & 88 & 74 & 111 & 93 & 74 & 108 & 78 & 76 & 80 \\
\hline d3-Estriol & -- & -- & -- & -- & -- & -- & -- & -- & -- & -- & -- & -- & -- & -- \\
\hline d4-Estrone & 124 & 118 & 138 & 140 & 106 & 69 & 76 & 89 & 82 & 54 & 95 & 67 & 82 & 65 \\
\hline d4-Mestranol & 89 & 90 & 100 & 118 & 90 & 41 & 59 & 70 & 64 & 49 & 78 & 55 & 65 & 50 \\
\hline d6-Norethindrone & 128 & 124 & 138 & 160 & 110 & 86 & 75 & 94 & 78 & 58 & 104 & 74 & 79 & 67 \\
\hline d9-Progesterone & 170 & 166 & 194 & 200 & 139 & 75 & 81 & 135 & 75 & 81 & 144 & 92 & 103 & 58 \\
\hline d5-Testosterone & 109 & 109 & 125 & 140 & 92 & 63 & 59 & 78 & 67 & 56 & 89 & 62 & 67 & 65 \\
\hline
\end{tabular}


Table 14. Summary of steroid and steroidal hormone compound data for passive organic compound integrative samplers (P0CIS) deployed in 11 Minnesota lakes during 2008.-Continued

[avg, average; $\mu \mathrm{g} / \mathrm{POCIS}$, microgram per POCIS; <, less than; Dup, duplicate sample; Trip, triplicate sample; --, not quantified]

\begin{tabular}{|c|c|c|c|c|c|c|c|c|c|}
\hline Compound & $\begin{array}{c}\text { Owasso } \\
\mu \mathrm{g} / \\
\text { POCIS }\end{array}$ & $\begin{array}{c}\text { Owasso Dup } \\
\mu g / \\
\text { POCIS }\end{array}$ & $\begin{array}{c}\text { Owasso Trip } \\
\mu g / \\
\text { POCIS }\end{array}$ & $\begin{array}{c}\text { Cedar } \\
\mu g / \\
\text { POCIS }\end{array}$ & $\begin{array}{c}\text { Cedar Dup } \\
\mu g / \\
\text { POCIS }\end{array}$ & $\begin{array}{c}\text { Cedar Trip } \\
\mu \mathrm{g} / \\
\text { POCIS }\end{array}$ & $\begin{array}{l}\text { Budd } \\
\mu g / \\
\text { POCIS }\end{array}$ & $\begin{array}{c}\text { Budd Dup } \\
\mu g / \\
\text { POCIS }\end{array}$ & $\begin{array}{c}\text { Budd Trip } \\
\mu \mathrm{g} / \\
\text { POCIS }\end{array}$ \\
\hline POCIS extracted & 1 & 1 & 1 & 1 & 1 & 1 & 1 & 1 & 1 \\
\hline 4-Androstene-3,17-dione & $<.0001$ & $<.0001$ & $<.0001$ & $<.0001$ & $<.0001$ & $<.0001$ & .0020 & .0022 & .0028 \\
\hline cis-Androsterone & $<.0001$ & $<.0001$ & $<.0001$ & $<.0001$ & $<.0001$ & $<.0001$ & $<.0001$ & $<.0001$ & $<.0001$ \\
\hline Cholesterol & $<.5$ & $<.5$ & $<.5$ & $<.5$ & $<.5$ & $<.5$ & 3.4 & 5.1 & 5.4 \\
\hline Coprostanol & $<.5$ & $<.5$ & $<.5$ & $<.5$ & $<.5$ & $<.5$ & $<.5$ & $<.5$ & $<.5$ \\
\hline Diethylstilbesterol & $<.0001$ & $<.0001$ & $<.0001$ & $<.0001$ & $<.0001$ & $<.0001$ & $<.0001$ & $<.0001$ & $<.0001$ \\
\hline Equilenin & $<.0001$ & $<.0001$ & $<.0001$ & $<.0001$ & $<.0001$ & $<.0001$ & $<.0001$ & $<.0001$ & $<.0001$ \\
\hline Equilin & $<.5$ & $<.5$ & $<.5$ & $<.5$ & $<.5$ & $<.5$ & $<.5$ & $<.5$ & $<.5$ \\
\hline $17 \alpha$-Estradiol & $<.0001$ & $<.0001$ & $<.0001$ & $<.0001$ & $<.0001$ & $<.0001$ & $<.0001$ & $<.0001$ & $<.0001$ \\
\hline $17 \beta$-Estradiol & .0004 & .0002 & .0007 & .0010 & .0004 & .0005 & .0002 & $<.0001$ & .0005 \\
\hline Estriol & $<.0001$ & $<.0001$ & $<.0001$ & $<.0001$ & $<.0001$ & $<.0001$ & $<.0001$ & $<.0001$ & $<.0001$ \\
\hline Estrone & .0014 & .0010 & .0014 & .0011 & .0014 & .0011 & .0010 & $<.0001$ & .0011 \\
\hline $17 \alpha$-Ethinylestradiol & $<.0001$ & $<.0001$ & $<.0001$ & $<.0001$ & $<.0001$ & $<.0001$ & $<.0001$ & $<.0001$ & $<.0001$ \\
\hline Mestranol & $<.0001$ & $<.0001$ & $<.0001$ & $<.0001$ & $<.0001$ & $<.0001$ & $<.0001$ & $<.0001$ & $<.0001$ \\
\hline Norethindrone & $<.0001$ & $<.0001$ & $<.0001$ & $<.0001$ & $<.0001$ & $<.0001$ & $<.0001$ & $<.0001$ & $<.0001$ \\
\hline Progesterone & $<.0001$ & $<.0001$ & $<.0001$ & $<.0001$ & $<.0001$ & $<.0001$ & $<.0001$ & $<.0001$ & $<.0001$ \\
\hline Testosterone & $<.0001$ & $<.0001$ & .0004 & $<.0001$ & $<.0001$ & $<.0001$ & $<.0001$ & .0003 & .0004 \\
\hline dihydro-Testosterone & $<.0001$ & $<.0001$ & $<.0001$ & $<.0001$ & $<.0001$ & $<.0001$ & $<.0001$ & $<.0001$ & $<.0001$ \\
\hline epi-Testosterone & $<.0001$ & $<.0001$ & $<.0001$ & $<.0001$ & $<.0001$ & $<.0001$ & $<.0001$ & $<.0001$ & $<.0001$ \\
\hline 11-keto-Testosterone & $<.0001$ & $<.0001$ & $<.0001$ & $<.0001$ & $<.0001$ & $<.0001$ & .0017 & $<.0001$ & $<.0001$ \\
\hline \multicolumn{10}{|c|}{ Surrogate Recovery (percent) } \\
\hline d7-Androstenedione & 87 & 160 & 160 & 85 & 139 & 173 & 107 & 153 & 168 \\
\hline d7-Cholesterol & 49 & 56 & 73 & 16 & 90 & 83 & 67 & 94 & 88 \\
\hline d8-Diethylstilbestrol & 46 & 119 & 118 & 62 & 82 & 108 & 62 & 100 & 113 \\
\hline d4-Ethinyl estradiol & 78 & 120 & 129 & 54 & 121 & 144 & 97 & 137 & 139 \\
\hline d4-Estradiol & 64 & 109 & 112 & 52 & 99 & 112 & 89 & 103 & 120 \\
\hline d3-Estriol & 0 & 0 & 0 & 0 & 0 & 0 & 0 & 0 & 0 \\
\hline d4-Estrone & 75 & 151 & 147 & 70 & 126 & 159 & 103 & 153 & 157 \\
\hline d4-Mestranol & 62 & 100 & 106 & 54 & 98 & 118 & 77 & 112 & 113 \\
\hline d6-Norethindrone & 88 & 143 & 153 & 69 & 137 & 166 & 107 & 151 & 155 \\
\hline d9-Progesterone & 114 & 189 & 206 & 66 & 205 & 228 & 139 & 226 & 219 \\
\hline d5-Testosterone & 72 & 122 & 133 & 66 & 115 & 145 & 93 & 134 & 147 \\
\hline
\end{tabular}


Table 15. Summary of field measurements for water samples collected from four Minnesota lakes during 2010.

$\left[{ }^{\circ} \mathrm{C}\right.$, degrees Celsius; $\mu \mathrm{S} / \mathrm{cm}$, microsiemens per centimeter; $\mathrm{mg} / \mathrm{L}$, milligrams per liter; std unit, standard unit]

\begin{tabular}{lcccc}
\hline & $\begin{array}{c}\text { Temperature } \\
{ }^{\circ} \mathbf{C}\end{array}$ & $\begin{array}{c}\text { Specific } \\
\text { conductance } \\
\boldsymbol{\mu S / c m}\end{array}$ & $\begin{array}{c}\text { Dissolved } \\
\mathbf{0 x y g e n} \\
\mathbf{m g} / \mathbf{L}\end{array}$ & $\begin{array}{c}\mathbf{p H} \\
\mathbf{s t d} \text { unit }\end{array}$ \\
\hline Elk & 11.8 & 294 & 8.6 & 8.4 \\
Beast & 10.7 & 23 & 9.5 & 6.9 \\
Bohall & 9.6 & 182 & 10.0 & 8.0 \\
Ryan & 10.7 & 23 & 9.8 & 6.8 \\
\hline
\end{tabular}

Table 16. Summary of acidic organic compound data for water samples collected from four Minnesota lakes during 2010.

$[\mu \mathrm{g} / \mathrm{L}$, microgram per liter; $<$, less than $]$

\begin{tabular}{lcccc}
\hline \multicolumn{1}{c}{ Compound } & $\begin{array}{c}\text { Elk } \\
\mathbf{\mu g} / \mathbf{L}\end{array}$ & $\begin{array}{c}\text { Beast } \\
\mathbf{\mu g} / \mathbf{L}\end{array}$ & $\begin{array}{c}\text { Bohall } \\
\mathbf{\mu g} / \mathbf{L}\end{array}$ & $\begin{array}{c}\text { Ryan } \\
\mathbf{\mu g} / \mathbf{L}\end{array}$ \\
\hline Ethylenediaminetetraacetic acid & 0.53 & $<0.05$ & 0.06 & 0.40 \\
Nitrilotriacetic acid & $<.05$ & $<.05$ & $<.05$ & $<.05$ \\
4-Nonylphenolmonoethoxycarboxylic acid & $<.1$ & $<.1$ & $<.1$ & $<.1$ \\
4-Nonylphenoldiethoxycarboxylic acid & $<.1$ & $<.1$ & $<.1$ & $<.1$ \\
4-Nonylphenoltriethoxycarboxylic acid & $<.1$ & $<.1$ & $<.1$ & $<.1$ \\
4-Nonylphenoltetraethoxycarboxylic acid & $<.1$ & $<.1$ & $<.1$ & $<.1$ \\
Surrogate Recovery (percent) & & & & \\
4-normal-Nonylphenoldiethoxycarboxylic acid & 61 & 59 & 67 & 54 \\
\hline
\end{tabular}


Table 17. Summary of neutral organic compound data for water samples collected from four Minnesota lakes during 2010.

[ $\mu \mathrm{g} / \mathrm{L}$, microgram per liter; $<$, less than; --, not quantifiable]

\begin{tabular}{|c|c|c|c|c|}
\hline Compound & $\begin{array}{c}\text { Elk } \\
\mu \mathrm{g} / \mathrm{L}\end{array}$ & $\begin{array}{c}\text { Beast } \\
\mu \mathrm{g} / \mathrm{L}\end{array}$ & $\begin{array}{c}\text { Bohall } \\
\mu \mathrm{g} / \mathrm{L}\end{array}$ & $\begin{array}{l}\text { Ryan } \\
\mu \mathrm{g} / \mathrm{L}\end{array}$ \\
\hline Acetylhexamethyltetrahydronaphthalene & $<0.01$ & $<0.01$ & $<0.01$ & $<0.01$ \\
\hline Bisphenol A & $<.01$ & $<.01$ & $<.01$ & $<.01$ \\
\hline 2[3]-tert-Butyl-4-methylphenol & $<.01$ & $<.01$ & $<.01$ & $<.01$ \\
\hline 4-tert-Butylphenol & $<.01$ & $<.01$ & $<.01$ & $<.01$ \\
\hline Caffeine & $<.01$ & $<.01$ & $<.01$ & $<.01$ \\
\hline 2,6-Di-tert-butyl-1,4-benzoquinone & .05 & .04 & .02 & $<.01$ \\
\hline 2,6-Di-tert-butyl-4-methylphenol & .01 & $<.01$ & $<.01$ & $<.01$ \\
\hline 2,6-Di-tert-butylphenol & $<.01$ & $<.01$ & $<.01$ & $<.01$ \\
\hline 1,2-Dichlorobenzene & $<.01$ & $<.01$ & $<.01$ & $<.01$ \\
\hline 1,3-Dichlorobenzene & $<.01$ & $<.01$ & $<.01$ & $<.01$ \\
\hline 1,4-Dichlorobenzene & $<.01$ & $<.01$ & $<.01$ & $<.01$ \\
\hline$N, N$-Diethyl-meta-toluamide & $<.01$ & $<.01$ & $<.01$ & $<.01$ \\
\hline 4-Ethylphenol & $<.01$ & $<.01$ & $<.01$ & $<.01$ \\
\hline Hexahydrohexamethylcyclopentabenzopyran & $<.01$ & $<.01$ & $<.01$ & $<.01$ \\
\hline 5-Methyl-1H-benzotriazole & $<.01$ & $<.01$ & $<.01$ & $<.01$ \\
\hline 4-Methylphenol & $<.01$ & .01 & $<.01$ & .01 \\
\hline 4-Nonylphenol & $<.05$ & $<.05$ & $<.05$ & $<.05$ \\
\hline 4-Nonylphenolmonoethoxylate & $<.05$ & $<.05$ & $<.05$ & $<.05$ \\
\hline 4-Nonylphenoldiethoxylate & $<.05$ & $<.05$ & $<.05$ & $<.05$ \\
\hline 4-normal-Octylphenol & $<.01$ & $<.01$ & $<.01$ & $<.01$ \\
\hline 4-tert-Octylphenol & $<.01$ & $<.01$ & $<.01$ & $<.01$ \\
\hline 4-tert-Octylphenolmonoethoxylate & $<.01$ & $<.01$ & $<.01$ & $<.01$ \\
\hline 4-tert-Octylphenoldiethoxylate & $<.01$ & $<.01$ & $<.01$ & $<.01$ \\
\hline 4-tert-Octylphenoltriethoxylate & $<.01$ & $<.01$ & $<.01$ & $<.01$ \\
\hline 4-tert-Octylphenoltetraethoxylate & $<.01$ & $<.01$ & $<.01$ & $<.01$ \\
\hline 4-tert-Octylphenolpentaethoxylate & $<.01$ & $<.01$ & $<.01$ & $<.01$ \\
\hline 4-tert-Pentylphenol & $<.01$ & $<.01$ & $<.01$ & $<.01$ \\
\hline 4-Propylphenol & $<.01$ & $<.01$ & $<.01$ & $<.01$ \\
\hline Triclosan & $<.01$ & $<.01$ & $<.01$ & $<.01$ \\
\hline \multicolumn{5}{|l|}{ Surrogate Recovery (percent) } \\
\hline d6-Bisphenol A & 27 & 49 & 52 & 32 \\
\hline $\mathrm{d} 21-2,6$-Di-tert-butyl-4-methylphenol & 53 & 85 & 21 & 63 \\
\hline 4-normal-Nonylphenol & 38 & 56 & 10 & 37 \\
\hline 4-normal-Nonylphenolmonoethoxylate & 38 & 51 & 47 & 37 \\
\hline 4-normal-Nonylphenoldiethoxylate & 46 & 63 & -- & 34 \\
\hline
\end{tabular}


Table 18. Summary of steroid and steroidal hormone compound data for water samples collected from four Minnesota lakes during 2010.

$[\mu \mathrm{g} / \mathrm{L}$, microgram per liter; $<$, less than]

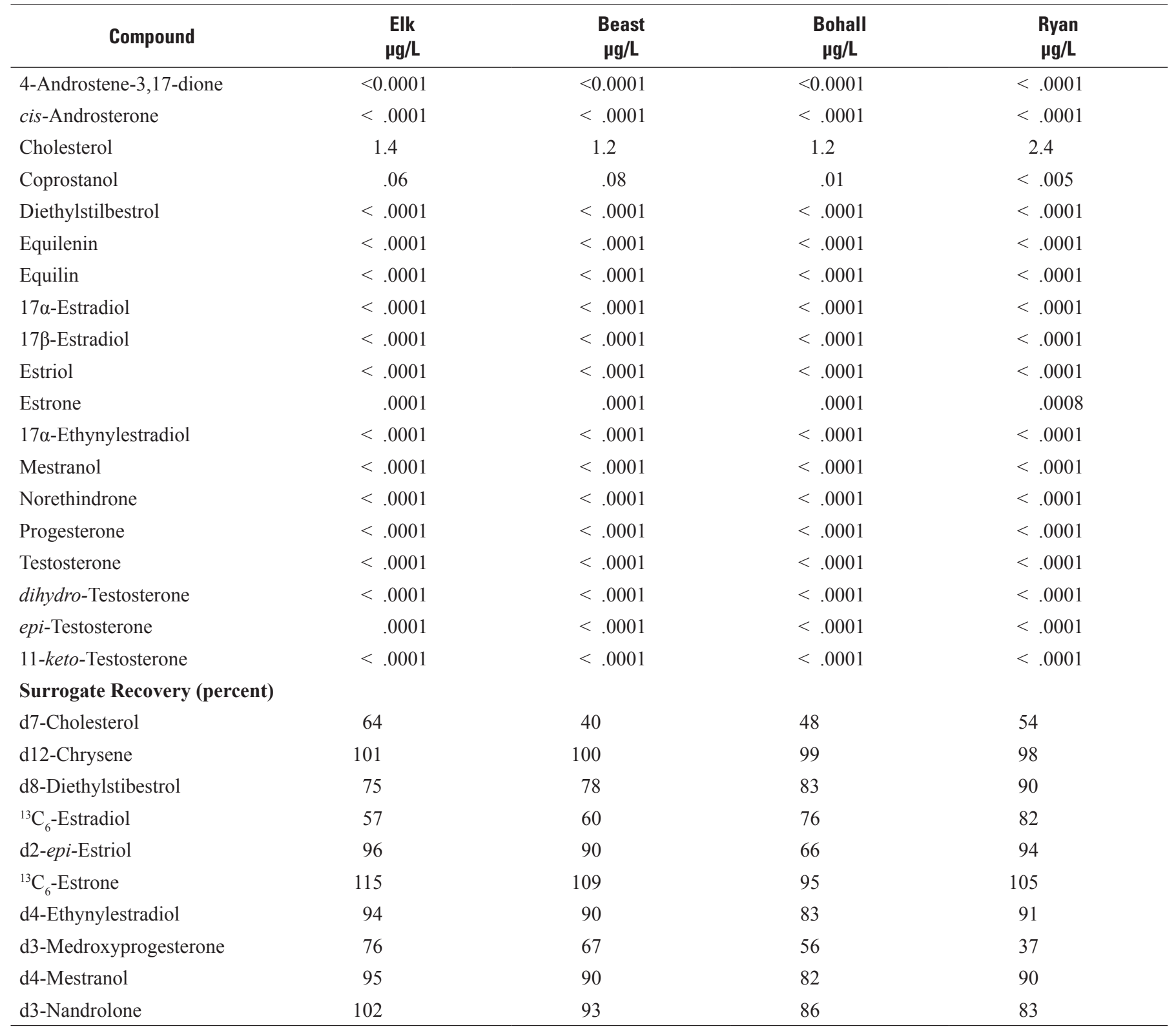


Table 19. Summary of field data for preliminary microhabitat selection at Sullivan Lake, Minn., June 1, 2010.

[Std unit, standard unit; $\mu \mathrm{S} / \mathrm{cm}$, microsiemens per centimeter; Temp, temperature; ${ }^{\circ} \mathrm{C}$, degrees Celsius; $\mathrm{mg} / \mathrm{L}$, milligrams per liter; NM, not measured; Temp residual $=$ Temp lake minus Temp pore; Temp residual less than $2^{\circ} \mathrm{C}$ indicates lake water loss downward to groundwater; Temp residual from $2^{\circ} \mathrm{C}$ to $5^{\circ} \mathrm{C}$ indicates mixing of groundwater and surface water; Temp residual greater than 5 indicates groundwater discharge to lake

\begin{tabular}{|c|c|c|c|c|c|c|c|c|c|}
\hline Location & Study site & Matrix & Lakebed & $\begin{array}{c}\mathrm{pH} \\
\text { std unit }\end{array}$ & $\begin{array}{c}\text { Specific } \\
\text { conductance } \\
\mu S / \mathrm{cm}\end{array}$ & Nitrate $\mathrm{mg} / \mathrm{L}$ & $\begin{array}{c}\text { Temp } \\
{ }^{\circ} \mathrm{C}\end{array}$ & $\begin{array}{c}\text { Temp } \\
\text { residual }\end{array}$ & $\begin{array}{c}\text { Assumed } \\
\text { water flow }\end{array}$ \\
\hline \multirow{5}{*}{$\begin{array}{l}\text { Surface water } \\
\text { and pore water } \\
\text { physio-chemical } \\
\text { characteristics of the } \\
\text { eastern shoreline } \\
\text { of Sullivan Lake } \\
\text { (from southwest } \\
\text { to northeast) }\end{array}$} & $\begin{array}{c}\text { Southwest } \\
\text { of site B }\end{array}$ & $\begin{array}{l}\text { Pore } \\
\text { Lake } \\
\text { Pore } \\
\text { Lake }\end{array}$ & $\begin{array}{l}\text { Silt/clay } \\
\text { Silt/clay } \\
\text { Silt/clay } \\
\text { Silt/clay }\end{array}$ & $\begin{array}{l}\mathrm{NM} \\
7.16 \\
\mathrm{NM} \\
7.89\end{array}$ & $\begin{array}{r}1,395 \\
416 \\
548 \\
379\end{array}$ & $\begin{array}{r}0.2 \\
.2 \\
.2 \\
.2\end{array}$ & $\begin{array}{l}18.6-19.1 \\
22.5 \\
16.0-16.9 \\
22.5\end{array}$ & $\begin{array}{l}3.4-3.9 \\
5.6-6.5\end{array}$ & $\begin{array}{l}\text { Mixing } \\
\text { Into Lake }\end{array}$ \\
\hline & Site B & $\begin{array}{l}\text { Pore } \\
\text { Lake } \\
\text { Pore } \\
\text { Lake } \\
\text { Pore } \\
\text { Lake } \\
\text { Pore } \\
\text { Pore } \\
\text { Pore } \\
\text { Lake } \\
\text { Pore }\end{array}$ & $\begin{array}{l}\text { Sandy } \\
\text { Sandy } \\
\text { Sandy } \\
\text { Sandy } \\
\text { Sandy } \\
\text { Sandy } \\
\text { Sandy } \\
\text { Sandy } \\
\text { Sandy } \\
\text { Sandy } \\
\text { Sandy }\end{array}$ & $\begin{array}{l}\text { NM } \\
7.87 \\
\text { NM } \\
8.19 \\
\text { NM } \\
7.85 \\
\text { NM } \\
\text { NM } \\
\text { NM } \\
7.75 \\
\text { NM }\end{array}$ & $\begin{array}{r}590 \\
403 \\
610 \\
402 \\
754 \\
424 \\
721 \\
871 \\
916 \\
400 \\
1,229\end{array}$ & $\begin{array}{c}\text { NM } \\
\text { NM } \\
.1 \\
\text { NM } \\
\text { NM } \\
\text { NM } \\
\text { NM } \\
\text { NM } \\
\text { NM } \\
.2 \\
\text { NM }\end{array}$ & $\begin{array}{c}16.6 \\
23.7 \\
21.4 \\
23.7 \\
\mathrm{NM} \\
23.7 \\
\mathrm{NM} \\
\mathrm{NM} \\
\mathrm{NM} \\
24.39 \\
\mathrm{NM}\end{array}$ & $\begin{array}{l}7.1 \\
2.3 \\
\text { NM } \\
\\
\mathrm{NM} \\
\mathrm{NM} \\
\mathrm{NM} \\
\mathrm{NM}\end{array}$ & $\begin{array}{l}\text { Into Lake } \\
\text { Mixing }\end{array}$ \\
\hline & Site A & $\begin{array}{l}\text { Pore } \\
\text { Pore } \\
\text { Pore } \\
\text { Pore } \\
\text { NM }\end{array}$ & $\begin{array}{l}\text { Sandy } \\
\text { Sandy } \\
\text { Sandy } \\
\text { Sandy } \\
\text { Silt/clay }\end{array}$ & $\begin{array}{l}\text { NM } \\
\text { NM } \\
\text { NM } \\
\text { NM } \\
\text { NM }\end{array}$ & $\begin{array}{r}876 \\
692 \\
678 \\
2,053 \\
\text { NM }\end{array}$ & $\begin{array}{c}.3 \\
.3 \\
.3 \\
.2 \\
\text { NM }\end{array}$ & $\begin{array}{l}\text { NM } \\
\text { NM } \\
\text { NM } \\
\text { NM } \\
\text { NM }\end{array}$ & $\begin{array}{l}\text { NM } \\
\text { NM } \\
\text { NM } \\
\text { NM } \\
\text { NM }\end{array}$ & \\
\hline & $\begin{array}{c}\text { Between sites } \\
\text { A and C }\end{array}$ & $\begin{array}{l}\text { Pore } \\
\text { Pore }\end{array}$ & $\begin{array}{l}\text { Silt/clay } \\
\text { Silt/clay }\end{array}$ & $\begin{array}{l}\mathrm{NM} \\
\mathrm{NM}\end{array}$ & $\begin{array}{l}1,868 \\
1,454\end{array}$ & $\begin{array}{l}4.6 \\
\mathrm{NM}\end{array}$ & $\begin{array}{l}\text { NM } \\
\text { NM }\end{array}$ & $\begin{array}{l}\mathrm{NM} \\
\mathrm{NM}\end{array}$ & \\
\hline & Site C & Lake & Silt/clay & 8.4 & 389 & NM & 24.3 & & \\
\hline
\end{tabular}


Table 20. Site characterization of Sullivan Lake microhabitat A (residential/septic influence) and water-quality values for pore-water samples collected July 8, 2010.

[x, distance parallel to shore; y, distance perpendicular to shore; see fig. 2 for specific sampling-grid orientation; $\mathrm{m}, \mathrm{meters;} \mathrm{mg} / \mathrm{L}, \mathrm{milligrams}$ per liter; $\mathrm{mm}$, millimeters; ${ }^{\circ} \mathrm{C}$, degrees Celsius; <, less than; NM, no field measurement; $\mathrm{cm}$, centimeters]

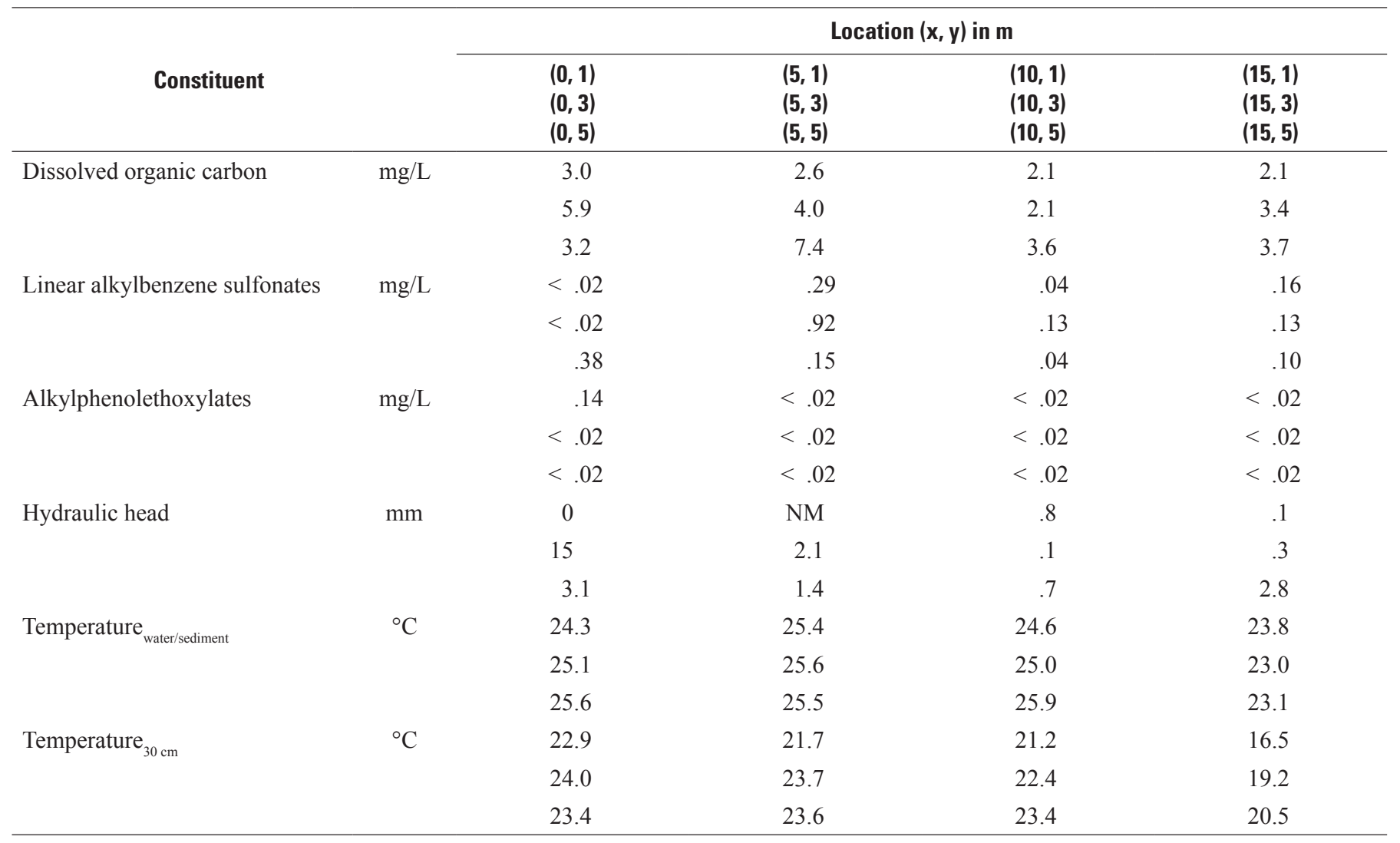

Table 21. Water column water-quality measurements for Sullivan Lake microhabitat A (residential/septic influence) collected from 1:30 to 3:00 PM on July 21, 2010.

[x, distance parallel to shore; y, distance perpendicular to shore; see fig. 2 for specific sampling-grid orientation; m, meters; total depth, distance from water surface to bed sediments; cm, centimeters; ${ }^{\circ} \mathrm{C}$, degrees Celsius; $\mu \mathrm{S} / \mathrm{cm}$, microsiemens per centimeter; mg/L, milligrams per liter; std unit, standard unit]

\begin{tabular}{|c|c|c|c|c|c|c|}
\hline Location $(x, y)$ in $m$ & $\begin{array}{l}\text { Total } \\
\text { depth } \\
\text { cm }\end{array}$ & $\begin{array}{c}\text { Depth from } \\
\text { water surface } \\
\text { cm }\end{array}$ & $\begin{array}{c}\text { Temperature } \\
{ }^{\circ} \mathrm{C}\end{array}$ & $\begin{array}{c}\text { Specific } \\
\text { conductance } \\
\mu \mathrm{S} / \mathrm{cm}\end{array}$ & $\begin{array}{c}\text { Dissolved } \\
\text { oxygen } \\
\text { mg/L }\end{array}$ & $\begin{array}{c}\mathrm{pH} \\
\text { std unit }\end{array}$ \\
\hline \multirow[t]{2}{*}{$(0,5)$} & 42 & 10 & 29.1 & 390 & 8.9 & 8.5 \\
\hline & & 32 & 28.9 & 392 & 7.7 & 8.3 \\
\hline \multirow[t]{2}{*}{$(3,5)$} & 39 & 10 & 29.1 & 388 & 7.9 & 8.6 \\
\hline & & 29 & 28.7 & 390 & 7.9 & 8.5 \\
\hline \multirow[t]{2}{*}{$(5,5)$} & 42 & 10 & 29.1 & 384 & 9.1 & 8.7 \\
\hline & & 32 & 30.0 & 385 & 8.8 & 8.6 \\
\hline \multirow[t]{2}{*}{$(10,5)$} & 44 & 10 & 29.1 & 386 & 7.8 & 8.6 \\
\hline & & 34 & 28.9 & 386 & 7.8 & 8.5 \\
\hline \multirow[t]{2}{*}{$(15,5)$} & 48 & 10 & 29.0 & 385 & 8.4 & 8.5 \\
\hline & & 38 & 27.4 & 414 & 5.8 & 7.9 \\
\hline
\end{tabular}


Table 22. Site characterization of Sullivan Lake microhabitat B (residential/septic influence) and water-quality values for pore-water samples collected July 8, 2010.

[x, distance parallel to shore; $\mathrm{y}$, distance perpendicular to shore; see figure 2 for specific sampling-grid orientation; $\mathrm{m}, \mathrm{meters} ; \mathrm{mg} / \mathrm{L}$, milligrams per liter; mm, millimeters; ${ }^{\circ} \mathrm{C}$, degrees Celsius; $<$, less than; NM, no field measurement; cm, centimeters]

\begin{tabular}{|c|c|c|c|c|c|}
\hline \multirow[b]{2}{*}{ Constituent } & & \multicolumn{4}{|c|}{ Location $(x, y)$ in $m$} \\
\hline & & $\begin{array}{l}\text { NM } \\
(0,3) \\
(0,5)\end{array}$ & $\begin{array}{l}\text { NM } \\
(5,3) \\
(5,5)\end{array}$ & $\begin{array}{l}(10,1) \\
(10,3) \\
(10,5)\end{array}$ & $\begin{array}{l}(15,1) \\
(15,3) \\
(15,5)\end{array}$ \\
\hline \multirow[t]{3}{*}{ Dissolved organic carbon } & $\mathrm{mg} / \mathrm{L}$ & NM & NM & 7.6 & 9.4 \\
\hline & & 7.8 & 7.6 & 8.7 & 10.4 \\
\hline & & 10.3 & 10.0 & 11.4 & 10.1 \\
\hline \multirow{2}{*}{ Linear alkylbenzene sulfonates } & & .02 & .04 & $<.02$ & $<.02$ \\
\hline & & .12 & .02 & $<.02$ & .03 \\
\hline \multirow[t]{3}{*}{ Alkylphenolethoxylates } & $\mathrm{mg} / \mathrm{L}$ & NM & NM & $<.02$ & $<.02$ \\
\hline & & $<.02$ & .10 & $<.02$ & .04 \\
\hline & & .11 & $<.02$ & $<.02$ & $<.02$ \\
\hline \multirow{3}{*}{ Temperature $_{\text {water/sediment }}$} & ${ }^{\circ} \mathrm{C}$ & NM & NM & NM & NM \\
\hline & & $\mathrm{NM}$ & $\mathrm{NM}$ & NM & NM \\
\hline & & NM & NM & NM & NM \\
\hline \multirow[t]{3}{*}{ Temperature $_{30 \mathrm{~cm}}$} & ${ }^{\circ} \mathrm{C}$ & NM & NM & NM & NM \\
\hline & & NM & $\mathrm{NM}$ & NM & NM \\
\hline & & $\mathrm{NM}$ & $\mathrm{NM}$ & $\mathrm{NM}$ & NM \\
\hline
\end{tabular}

Table 23. Water column water-quality measurements for Sullivan Lake microhabitat B (residential/septic influence) collected 10:30 AM to 2:00 PM on July 21, 2010.

[x, distance parallel to shore; y, distance perpendicular to shore; see fig. 2 for specific sampling-grid orientation; m, meters; total depth, distance from water surface to bed sediments; $\mathrm{cm}$, centimeters; ${ }^{\circ} \mathrm{C}$, degrees Celsius; $\mu \mathrm{S} / \mathrm{cm}$, microsiemens per centimeter; mg/L, milligrams per liter; std unit, standard unit]

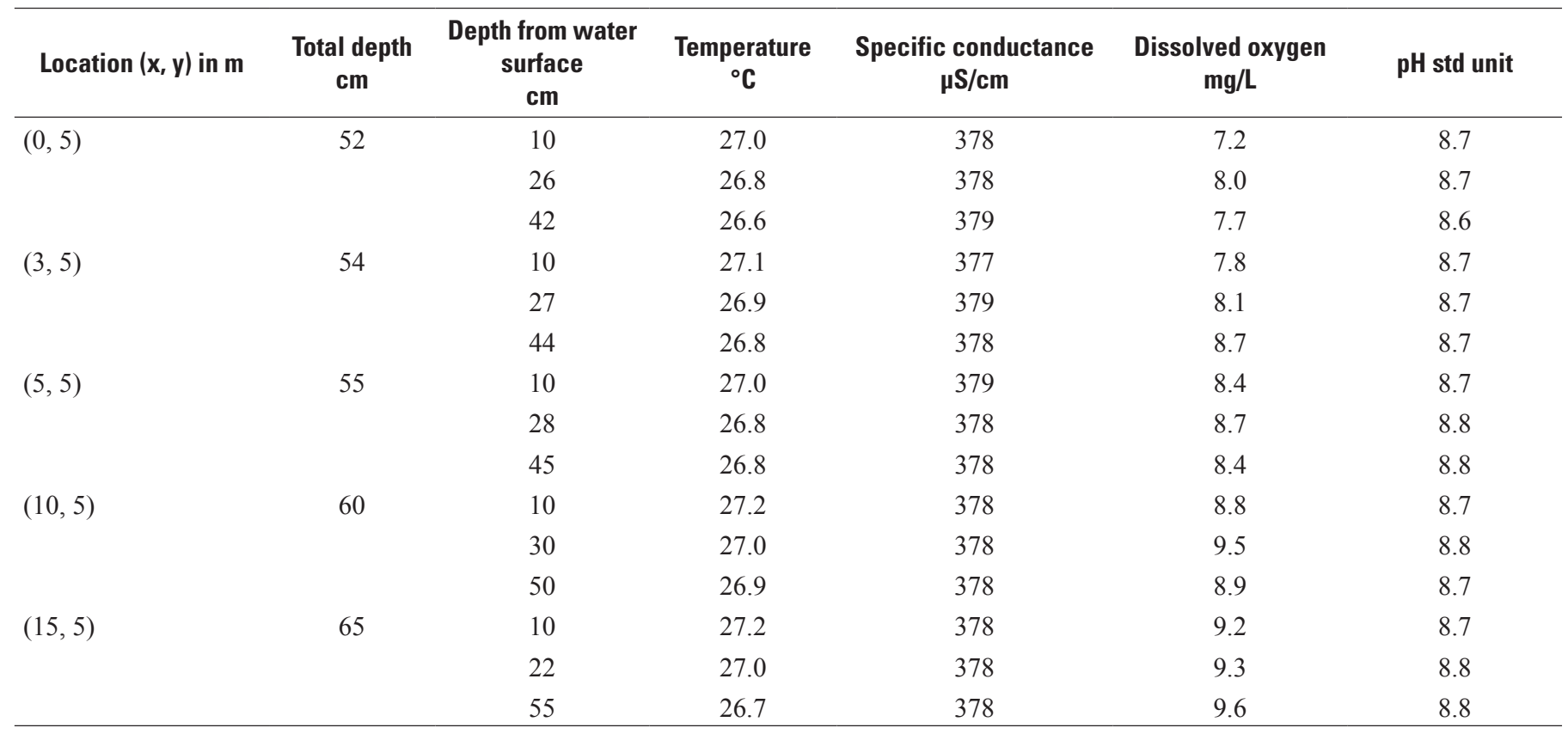


Table 24. Site characterization of Sullivan Lake microhabitat C (stormwater/boat-ramp influence) and water-quality values for pore-water samples collected July 8, 2010.

[x, distance parallel to shore; $\mathrm{y}$, distance perpendicular to shore; see fig. 2 for specific sampling-grid orientation; $\mathrm{m}$, meters; $\mathrm{mg} / \mathrm{L}$, milligrams per liter; ${ }^{\circ} \mathrm{C}$, degrees Celsius; <, less than; $\mathrm{NM}$, not measured; cm, centimeters]

\begin{tabular}{lccc}
\hline \multirow{2}{*}{ Constituent } & & \multicolumn{2}{c}{ Location $(\mathbf{x}, \mathbf{y})$ in $\mathbf{~ m}$} \\
\cline { 3 - 4 } & & $\mathbf{( 0 , 1 )}$ & $\mathbf{N M}$ \\
& & $\mathbf{N M}$ & $(\mathbf{1 5}, \mathbf{5})$ \\
\hline Dissolved organic carbon & $\mathrm{mg} / \mathrm{L}$ & 11.2 & $\mathrm{NM}$ \\
Linear alkylbenzene sulfonates & $\mathrm{mg} / \mathrm{L}$ & $\mathrm{NM}$ & 7.3 \\
& & $<.02$ & $\mathrm{NM}$ \\
Alkylphenolethoxylates & $\mathrm{mg} / \mathrm{L}$ & $\mathrm{NM}$ & .03 \\
& & .11 & $\mathrm{NM}$ \\
Temperature & & $\mathrm{NM}$ & $<.02$ \\
& & 23.7 & $\mathrm{NM}$ \\
Tempersediment $^{\circ} \mathrm{C}$ & $\mathrm{NM}$ & 28.6 \\
& & 19.4 & $\mathrm{NM}$ \\
\hline
\end{tabular}

Table 25. Water column water-quality measurements for Sullivan Lake microhabitat C (stormwater/boat ramp influence) collected 12:00 to 1:30 PM on July 21, 2010.

[x, distance parallel to shore; y, distance perpendicular to shore; see fig. 2 for specific sampling-grid orientation; m, meters; total depth, distance from water surface to bed sediments; $\mathrm{cm}$, centimeters; ${ }^{\circ} \mathrm{C}$, degrees Celsius; $\mu \mathrm{S} / \mathrm{cm}$, microsiemens per centimeter; $\mathrm{mg} / \mathrm{L}$, milligrams per liter; std unit, standard unit]

\begin{tabular}{|c|c|c|c|c|c|c|}
\hline Location $(x, y)$ in $m$ & $\begin{array}{l}\text { Total depth } \\
\text { cm }\end{array}$ & $\begin{array}{l}\text { Depth from water } \\
\text { surface } \mathrm{cm}\end{array}$ & Temperature ${ }^{\circ} \mathrm{C}$ & $\begin{array}{c}\text { Specific conductance } \\
\mu \mathrm{S} / \mathrm{cm}\end{array}$ & $\begin{array}{c}\text { Dissolved oxygen } \\
\mathrm{mg} / \mathrm{L}\end{array}$ & $\begin{array}{c}\mathrm{pH} \text { std } \\
\text { unit }\end{array}$ \\
\hline \multirow[t]{3}{*}{$(0,5)$} & 82 & 10 & 27.1 & 384 & 9.2 & 8.4 \\
\hline & & 41 & 26.3 & 387 & 7.8 & 8.1 \\
\hline & & 72 & 26.2 & 392 & 5.2 & 7.8 \\
\hline \multirow[t]{3}{*}{$(3,5)$} & 69 & 10 & 27.4 & 383 & 7.7 & 8.4 \\
\hline & & 35 & 26.8 & 385 & 7.8 & 8.3 \\
\hline & & 59 & 26.3 & 384 & 7.5 & 8.3 \\
\hline \multirow[t]{3}{*}{$(5,5)$} & 68 & 10 & 27.4 & 381 & 8.4 & 8.4 \\
\hline & & 34 & 26.6 & 384 & 7.8 & 8.2 \\
\hline & & 58 & 26.2 & 389 & 6.9 & 7.7 \\
\hline \multirow[t]{3}{*}{$(10,5)$} & 73 & 10 & 27.5 & 379 & 9.6 & 8.5 \\
\hline & & 37 & 26.5 & 384 & 9.1 & 8.1 \\
\hline & & 63 & 26.3 & 388 & 7.3 & 7.8 \\
\hline \multirow[t]{3}{*}{$(15,5)$} & 71 & 10 & 26.9 & 379 & 10.1 & 8.5 \\
\hline & & 36 & 26.5 & 384 & 8.0 & 8.1 \\
\hline & & 61 & 26.1 & 394 & 5.9 & 7.5 \\
\hline
\end{tabular}


Table 26. Site characterization of Sullivan Lake microhabitat D (agricultural influence) and water-quality values for pore-water samples collected July 9, 2010.

[x, distance parallel to shore; $\mathrm{y}$, distance perpendicular to shore; see fig. 2 for specific sampling-grid orientation; $\mathrm{m}$, meters; mg/L, milligrams per liter; mm, millimeters; ${ }^{\circ} \mathrm{C}$, degrees Celsius; $<$, less than; NM, no measurement; * positive bias due to high inorganic carbon content; cm, centimeters]

\begin{tabular}{|c|c|c|c|}
\hline \multirow{2}{*}{ Constituent } & & \multicolumn{2}{|c|}{ Location $(x, y)$ in $m$} \\
\hline & & $(0,1)$ & NM \\
\hline \multirow{4}{*}{ Dissolved organic carbon } & & & \\
\hline & $\mathrm{mg} / \mathrm{L}$ & $31.8^{*}$ & NM \\
\hline & & $12.6^{*}$ & $\mathrm{NM}$ \\
\hline & & $32.3^{*}$ & 5.0 \\
\hline \multirow[t]{3}{*}{ Linear alkylbenzene sulfonates } & $\mathrm{mg} / \mathrm{L}$ & .02 & NM \\
\hline & & .11 & NM \\
\hline & & .02 & .11 \\
\hline \multirow[t]{3}{*}{ Alkylphenolethoxylates } & $\mathrm{mg} / \mathrm{L}$ & $<.02$ & NM \\
\hline & & $<.02$ & NM \\
\hline & & $<.02$ & $<.02$ \\
\hline \multirow[t]{3}{*}{ Hydraulic head } & $\mathrm{mm}$ & 1.2 & $\mathrm{NM}$ \\
\hline & & NM & NM \\
\hline & & $\mathrm{NM}$ & NM \\
\hline \multirow[t]{3}{*}{ Temperature $_{\text {water sediment }}$} & ${ }^{\circ} \mathrm{C}$ & 22.9 & NM \\
\hline & & 23.8 & $\mathrm{NM}$ \\
\hline & & 21.7 & 19.9 \\
\hline \multirow[t]{3}{*}{ Temperature $_{30 \mathrm{~cm}}$} & ${ }^{\circ} \mathrm{C}$ & 18.5 & $\mathrm{NM}$ \\
\hline & & 18.8 & NM \\
\hline & & 17.4 & 16.7 \\
\hline
\end{tabular}

Table 27. Water column water-quality measurements for Sullivan Lake microhabitat D (agricultural influence) collected 9:00 to 10:30 AM on July 21, 2010.

[x, distance parallel to shore; $y$, distance perpendicular to shore; see fig. 2 for specific sampling-grid orientation; $\mathrm{m}$, meters; cm, centimeters; ${ }^{\circ} \mathrm{C}$, degrees Celsius; $\mathrm{mS} / \mathrm{cm}$, microsiemens per centimeter; $\mathrm{mg} / \mathrm{L}$, milligrams per liter; std unit, standard unit; NM, no measurement]

\begin{tabular}{|c|c|c|c|c|c|c|}
\hline Location $(x, y)$ in $m$ & $\begin{array}{c}\text { Total depth } \\
\text { cm }\end{array}$ & $\begin{array}{l}\text { Depth from water } \\
\text { surface } \mathbf{c m}\end{array}$ & Temperature ${ }^{\circ} \mathrm{C}$ & $\begin{array}{c}\text { Specific } \\
\text { conductance } \mu \mathrm{S} / \mathrm{cm}\end{array}$ & $\begin{array}{c}\text { Dissolved } \\
\text { oxygen mg/L }\end{array}$ & pH std unit \\
\hline \multirow[t]{3}{*}{$(0,5)$} & 70 & 10 & 25.1 & 388 & 4.1 & 7.4 \\
\hline & & 35 & 25.2 & 388 & 3.7 & 7.5 \\
\hline & & 50 & 25.1 & 389 & 3.6 & 7.5 \\
\hline \multirow[t]{3}{*}{$(3,5)$} & 72 & 10 & 25.5 & 880 & 4.3 & 7.6 \\
\hline & & 36 & 25.1 & 406 & 3.2 & 6.9 \\
\hline & & 52 & 25.0 & 443 & 2.9 & 6.7 \\
\hline \multirow[t]{3}{*}{$(5,5)$} & 76 & 10 & 25.4 & 389 & 4.4 & 7.5 \\
\hline & & 38 & 25.2 & 450 & 2.9 & 6.7 \\
\hline & & 56 & 25.0 & 540 & 1.9 & 6.7 \\
\hline \multirow[t]{3}{*}{$(10,5)$} & 71 & 10 & 25.8 & 385 & 5.1 & 7.9 \\
\hline & & 36 & 25.4 & 472 & 2.5 & 6.7 \\
\hline & & 51 & NM & 252 & 1.9 & 6.6 \\
\hline \multirow[t]{3}{*}{$(15,5)$} & 78 & 10 & 26.1 & 384 & 5.6 & 7.9 \\
\hline & & 39 & 25.6 & 387 & 4.8 & 7.7 \\
\hline & & 58 & 25.2 & 451 & 3.0 & 6.8 \\
\hline
\end{tabular}


Table 28. Summary of acidic organic compound data for water samples collected from four microhabitat sites in Sullivan Lake, Minn., on July 8, 2010.

[Microhabitats A and B, residential/septic influenced; microhabitat C, stormwater/boat-ramp influenced; microhabitat D, agriculture influenced; $\mu$ g/L, microgram per liter; <, less than; Dup, duplicate sample; MS, matrix spike recovery in percent; NM, no measurement]

\begin{tabular}{|c|c|c|c|c|c|c|c|c|c|c|c|c|c|}
\hline Compound & $\begin{array}{c}\text { Site } \\
\text { A } \\
\mu \mathrm{g} / \mathrm{L}\end{array}$ & $\begin{array}{c}\text { Site } \\
\text { A-Dup } \\
\mu g / L\end{array}$ & $\begin{array}{c}\text { Site } \\
\text { A-MS } \\
\text { Percent }\end{array}$ & $\begin{array}{c}\text { Site } \\
\text { B } \\
\mu \mathrm{g} / \mathrm{L}\end{array}$ & $\begin{array}{c}\text { Site } \\
\text { B-Dup } \\
\mu \mathrm{g} / \mathrm{L}\end{array}$ & $\begin{array}{c}\text { Site } \\
\text { C } \\
\mu \mathrm{g} / \mathrm{L}\end{array}$ & $\begin{array}{c}\text { Site } \\
\text { C-Dup } \\
\mu \mathrm{g} / \mathrm{L}\end{array}$ & $\begin{array}{c}\text { Site } \\
\text { C-MS } \\
\text { percent }\end{array}$ & $\begin{array}{c}\text { Site } \\
\text { D } \\
\mu \mathrm{g} / \mathrm{L}\end{array}$ & $\begin{array}{l}\text { Site } \\
\text { D-Dup } \\
\mu g / L\end{array}$ & $\begin{array}{c}\text { Blank } \\
\mu \mathrm{g} / \mathrm{L}\end{array}$ & $\begin{array}{c}\text { Blank } \\
\mu \mathrm{g} / \mathrm{L}\end{array}$ & $\begin{array}{c}\text { Blank } \\
\mu \mathrm{g} / \mathrm{L}\end{array}$ \\
\hline Ethylenediaminetetraacetic acid & 0.2 & 0.2 & 63 & 0.2 & 0.2 & 0.2 & 0.2 & 68.6 & 0.6 & 0.6 & $<0.05$ & $<0.05$ & $<0.05$ \\
\hline Nitrilotriacetic acid & .1 & .1 & 101 & .1 & .1 & .1 & .1 & 98.4 & .1 & .1 & $<.05$ & $<.05$ & $<.05$ \\
\hline 4-Nonylphenolmonoethoxycarboxylic acid & 6 & .4 & 90 & .4 & .4 & .4 & .5 & 90.9 & .4 & .4 & $<.1$ & $<.1$ & $<.1$ \\
\hline 4-Nonylphenoldiethoxycarboxylic acid & .5 & .1 & 95 & $<.1$ & $<.1$ & $<.1$ & $<.1$ & 90.0 & $<.1$ & $<.1$ & $<.1$ & $<.1$ & $<.1$ \\
\hline 4-Nonylphenoltriethoxycarboxylic acid & $<.1$ & $<.1$ & 80 & $<.1$ & $<.1$ & $<.1$ & $<.1$ & 78.1 & $<.1$ & $<.1$ & $<.1$ & $<.1$ & $<.1$ \\
\hline 4-Nonylphenoltetraethoxycarboxylic acid & $<.1$ & $<.1$ & 84 & $<.1$ & $<.1$ & $<.1$ & $<.1$ & 80.0 & $<.1$ & $<.1$ & $<.1$ & $<.1$ & $<.1$ \\
\hline \multicolumn{14}{|l|}{ Surrogate Recovery (percent) } \\
\hline 4-normal-Nonylphenoldiethoxycarboxylic acid & 69 & 66 & 95 & 68 & 72 & 68 & 87 & 85 & 79 & 75 & 98 & NM & 75 \\
\hline
\end{tabular}

Table 29. Summary of acidic organic compound data for water samples collected from four microhabitat sites in Sullivan Lake, Minn., on 0ctober 12, 2010.

[Microhabitats A and B, residential/septic influenced; microhabitat C, stormwater/boat-ramp influenced; microhabitat D, agriculture influenced; $\mu \mathrm{g} / \mathrm{L}$, microgram per liter; <, less than; Dup, duplicate sample]

\begin{tabular}{|c|c|c|c|c|c|}
\hline Compound & $\begin{array}{c}\text { Site } \\
\text { A } \\
\mu \mathrm{g} / \mathrm{L}\end{array}$ & $\begin{array}{c}\text { Site } \\
\text { A Dup } \\
\mu \mathrm{g} / \mathrm{L}\end{array}$ & $\begin{array}{c}\text { Site } \\
\text { B } \\
\mu \mathrm{g} / \mathrm{L}\end{array}$ & $\begin{array}{c}\text { Site } \\
\text { C } \\
\mu \mathrm{g} / \mathrm{L}\end{array}$ & $\begin{array}{c}\text { Site } \\
\text { D } \\
\mu \mathrm{g} / \mathrm{L}\end{array}$ \\
\hline Ethylenediaminetetraacetic acid & 0.3 & 0.3 & 0.2 & 0.2 & 0.3 \\
\hline Nitrilotriacetic acid & .1 & .1 & $<.05$ & $<.05$ & $<.05$ \\
\hline 4-Nonylphenolmonoethoxycarboxylic acid & $<.1$ & $<.1$ & $<.1$ & $<.1$ & $<.1$ \\
\hline 4-Nonylphenoldiethoxycarboxylic acid & .2 & .1 & $<.1$ & $<.1$ & $<.1$ \\
\hline 4-Nonylphenoltriethoxycarboxylic acid & $<.1$ & $<.1$ & $<.1$ & $<.1$ & $<.1$ \\
\hline 4-Nonylphenoltetraethoxycarboxylic acid & $<.1$ & $<.1$ & $<.1$ & $<.1$ & $<.1$ \\
\hline \multicolumn{6}{|l|}{ Surrogate Recovery (percent) } \\
\hline 4-normal-Nonylphenoldiethoxycarboxylic acid & 64 & 46 & 55 & 70 & 52 \\
\hline
\end{tabular}


Table 30. Summary of neutral organic compound data for water samples collected from four microhabitat sites in Sullivan Lake, Minn. on July 8, 2010.

[Microhabitats A and B, residential/septic influenced; microhabitat C, stormwater/boat-ramp influenced; microhabitat D, agriculture influenced; $\mu \mathrm{g} / \mathrm{L}$, microgram per liter; <, less than; Dup, duplicate sample; MS, matrix spike recovery in percent; NM, not measured]

\begin{tabular}{|c|c|c|c|c|c|c|c|c|c|c|c|c|}
\hline Compound & $\begin{array}{c}\text { Site } \\
\text { A } \\
\mu \mathrm{g} / \mathrm{L}\end{array}$ & $\begin{array}{l}\text { Site } \\
\text { A-Dup } \\
\mu \mathrm{g} / \mathrm{L}\end{array}$ & $\begin{array}{c}\text { Site } \\
\text { A-MS } \\
\text { percent }\end{array}$ & $\begin{array}{c}\text { Site } \\
\text { B } \\
\mu \mathrm{g} / \mathrm{L}\end{array}$ & $\begin{array}{c}\text { Site } \\
\text { B-Dup } \\
\mu \mathrm{g} / \mathrm{L}\end{array}$ & $\begin{array}{c}\text { Site } \\
\text { B-MS } \\
\text { percent }\end{array}$ & $\begin{array}{c}\text { Site } \\
\text { C } \\
\mu \mathrm{g} / \mathrm{L}\end{array}$ & $\begin{array}{l}\text { Site } \\
\text { C-Dup } \\
\mu \mathrm{g} / \mathrm{L}\end{array}$ & $\begin{array}{c}\text { Site } \\
\text { C-MS } \\
\text { percent }\end{array}$ & $\begin{array}{c}\text { Site } \\
D \\
\mu \mathrm{g} / \mathrm{L}\end{array}$ & $\begin{array}{l}\text { Site } \\
\text { D-Dup } \\
\mu \mathrm{g} / \mathrm{L}\end{array}$ & $\begin{array}{c}\text { Site } \\
\text { D-MS } \\
\text { percent }\end{array}$ \\
\hline Acetylhexamethyltetrahydronaphthalene & $<0.01$ & $<0.01$ & 111 & $<0.01$ & $<0.01$ & 114 & $<0.01$ & $<0.01$ & 131 & $<0.01$ & $<0.01$ & 124 \\
\hline Bisphenol A & .03 & .02 & 67 & .05 & .01 & 68 & $<.01$ & $<.01$ & 85 & $<.01$ & $<.01$ & 84 \\
\hline 2[3]-tert-Butyl-4-methylphenol & $<.01$ & $<.01$ & 118 & $<.01$ & $<.01$ & 92 & $<.01$ & $<.01$ & 131 & $<.01$ & $<.01$ & 123 \\
\hline 4-tert-butylphenol & $<.01$ & $<.01$ & 111 & $<.01$ & $<.01$ & 116 & $<.01$ & $<.01$ & 129 & $<.01$ & $<.01$ & 129 \\
\hline Caffeine & .01 & .02 & 31 & $<.01$ & $<.01$ & 31 & $<.01$ & $<.01$ & 30 & $<.01$ & $<.01$ & 41 \\
\hline 2,6-Di-tert-butyl-1,4-benzoquinone & .03 & .03 & NM & .04 & .03 & NM & .04 & .03 & NM & .03 & .03 & NM \\
\hline 2,6-Di-tert-butyl-4-methylphenol & .04 & .02 & NM & .02 & .01 & NM & .03 & .04 & NM & .02 & $<.01$ & NM \\
\hline 2,6-Di-tert-butylphenol & $<.01$ & $<.01$ & 136 & $<.01$ & $<.01$ & 98 & $<.01$ & $<.01$ & 146 & $<.01$ & $<.01$ & 143 \\
\hline 1,2-Dichlorobenzene & $<.01$ & $<.01$ & 99 & $<.01$ & $<.01$ & 98 & $<.01$ & $<.01$ & 88 & $<.01$ & $<.01$ & 111 \\
\hline 1,3-Dichlorobenzene & $<.01$ & $<.01$ & 98 & $<.01$ & $<.01$ & 96 & $<.01$ & $<.01$ & 88 & $<.01$ & $<.01$ & 110 \\
\hline 1,4-Dichlorobenzene & $<.01$ & $<.01$ & 98 & $<.01$ & $<.01$ & 96 & $<.01$ & $<.01$ & 87 & $<.01$ & $<.01$ & 110 \\
\hline$N, N$-Diethyl-meta-toluamide & .15 & .14 & 107 & .13 & .13 & 118 & .07 & .08 & 117 & .06 & .06 & 125 \\
\hline 4-Ethylphenol & $<.01$ & $<.01$ & 73 & $<.01$ & $<.01$ & 69 & .01 & $<.01$ & 68 & $<.01$ & $<.01$ & 84 \\
\hline Hexahydrohexamethylcyclopentabenzopyran & $<.01$ & $<.01$ & 107 & $<.01$ & $<.01$ & 107 & $<.01$ & $<.01$ & 124 & $<.01$ & $<.01$ & 118 \\
\hline 5-Methyl-1H-Benzotriazole & $<.01$ & $<.01$ & $<10$ & $<.01$ & $<.01$ & $<10$ & $<.01$ & $<.01$ & $<10$ & $<.01$ & $<.01$ & $<10$ \\
\hline 4-Methylphenol & .02 & .03 & 43 & .03 & .03 & 39 & .03 & .04 & 41 & .03 & .03 & 54 \\
\hline 4-Nonylphenol & .09 & .09 & 122 & .05 & .05 & 131 & $<.05$ & $<.05$ & 163 & $<.05$ & $<.05$ & 159 \\
\hline 4-Nonylphenolmonoethoxylate & .28 & .09 & 134 & .09 & .08 & 137 & $<.05$ & $<.05$ & 161 & $<.05$ & $<.05$ & 152 \\
\hline 4-Nonylphenoldiethoxylate & $<.05$ & $<.05$ & 110 & $<.05$ & $<.05$ & 117 & $<.05$ & $<.05$ & 137 & $<.05$ & $<.05$ & 129 \\
\hline 4-normal-Octylphenol & $<.01$ & $<.01$ & 12 & $<.01$ & $<.01$ & 12 & $<.01$ & $<.01$ & 15 & $<.01$ & $<.01$ & 14 \\
\hline 4-tert-Octylphenol & .04 & .02 & 137 & .02 & .01 & 138 & .02 & .01 & 162 & $<.01$ & $<.01$ & 157 \\
\hline 4-tert-Octylphenolmonoethoxylate & .02 & $<.01$ & 113 & $<.01$ & $<.01$ & 115 & $<.01$ & $<.01$ & 133 & $<.01$ & $<.01$ & 128 \\
\hline 4-tert-Octylphenoldiethoxylate & $<.01$ & $<.01$ & 109 & $<.01$ & $<.01$ & 108 & $<.01$ & $<.01$ & 122 & $<.01$ & $<.01$ & 119 \\
\hline 4-tert-Octylphenoltriethoxylate & $<.01$ & $<.01$ & 95 & $<.01$ & $<.01$ & 83 & $<.01$ & $<.01$ & 91 & $<.01$ & $<.01$ & 91 \\
\hline 4-tert-Octylphenoltetraethoxylate & $<.01$ & $<.01$ & 70 & $<.01$ & $<.01$ & 72 & $<.01$ & $<.01$ & 73 & $<.01$ & $<.01$ & 101 \\
\hline 4-tert-Octylphenolpentaethoxylate & $<.01$ & $<.01$ & 47 & $<.01$ & $<.01$ & 34 & $<.01$ & $<.01$ & 45 & $<.01$ & $<.01$ & 55 \\
\hline 4-tert-Pentylphenol & $<.01$ & $<.01$ & 122 & $<.01$ & $<.01$ & 128 & $<.01$ & $<.01$ & 147 & $<.01$ & $<.01$ & 142 \\
\hline 4-Propylphenol & $<.01$ & $<.01$ & 115 & $<.01$ & $<.01$ & 114 & $<.01$ & $<.01$ & 122 & $<.01$ & $<.01$ & 128 \\
\hline Triclosan & $<.01$ & $<.01$ & 100 & $<.01$ & $<.01$ & 87 & $<.01$ & $<.01$ & 100 & $<.01$ & $<.01$ & 102 \\
\hline \multicolumn{13}{|l|}{ Surrogate Recovery (percent) } \\
\hline d6-Bisphenol A & 72 & 78 & & 78 & 77 & & 96 & 99 & & 85 & 81 & \\
\hline d21-2,6-Di-tert-butyl-4-methylphenol & 49 & 71 & & 59 & 61 & & 60 & 89 & & 65 & 70 & \\
\hline 4-normal-Nonylphenol & 52 & 57 & & 53 & 52 & & 69 & 76 & & 55 & 53 & \\
\hline 4-normal-Nonylphenolmonoethoxylate & 47 & 52 & & 52 & 51 & & 74 & 77 & & 56 & 53 & \\
\hline 4-normal-Nonylphenoldiethoxylate & 29 & 39 & & 41 & 40 & & 59 & 63 & & 45 & 40 & \\
\hline
\end{tabular}


Table 31. Summary of neutral organic compound data for water samples collected from four microhabitat sites in Sullivan Lake, Minn., on 0ctober 12, 2010.

[Microhabitats A and B, residential/septic influenced; microhabitat C, stormwater/boat ramp influenced; microhabitat D, agriculture influenced; $\mu \mathrm{g} / \mathrm{L}$, microgram per liter; <, less than; Dup, duplicate sample]

\begin{tabular}{|c|c|c|c|c|c|c|c|}
\hline Compound & $\begin{array}{c}\text { Site A } \\
\mu \mathrm{g} / \mathrm{L}\end{array}$ & $\begin{array}{c}\text { Site A-Dup } \\
\mu \mathrm{g} / \mathrm{L}\end{array}$ & $\begin{array}{c}\text { Site B } \\
\mu \mathrm{g} / \mathrm{L}\end{array}$ & $\begin{array}{c}\text { Site B-Dup } \\
\mu \mathrm{g} / \mathrm{L}\end{array}$ & $\begin{array}{c}\text { Site C } \\
\mu \mathrm{g} / \mathrm{L}\end{array}$ & $\begin{array}{c}\text { Site D } \\
\mu \mathrm{g} / \mathrm{L}\end{array}$ & $\begin{array}{c}\text { Site D-Dup } \\
\mu \mathrm{g} / \mathrm{L}\end{array}$ \\
\hline Acetylhexamethyltetrahydronaphthalene & 0.02 & 0.01 & 0.01 & 0.02 & $<0.01$ & $<0.01$ & $<0.01$ \\
\hline Bisphenol A & .01 & .01 & .15 & .02 & $<.01$ & $<.01$ & $<.01$ \\
\hline 2[3]-tert-Butyl-4-methylphenol & $<.01$ & $<.01$ & $<.01$ & $<.01$ & $<.01$ & $<.01$ & $<.01$ \\
\hline 4-tert-Butylphenol & $<.01$ & $<.01$ & $<.01$ & $<.01$ & $<.01$ & $<.01$ & $<.01$ \\
\hline Caffeine & .02 & .05 & .04 & .01 & $<.01$ & $<.01$ & $<.01$ \\
\hline 2,6-Di-tert-butyl-1,4-benzoquinone & .06 & .09 & .04 & .06 & .04 & $<.01$ & .04 \\
\hline 2,6-Di-tert-butyl-4-methylphenol & .01 & .01 & $<.01$ & .01 & .01 & .02 & .02 \\
\hline 2,6-Di-tert-butylphenol & $<.01$ & $<.01$ & $<.01$ & $<.01$ & $<.01$ & $<.01$ & $<.01$ \\
\hline 1,2-Dichlorobenzene & $<.01$ & $<.01$ & $<.01$ & $<.01$ & $<.01$ & $<.01$ & $<.01$ \\
\hline 1,3-Dichlorobenzene & $<.01$ & $<.01$ & $<.01$ & $<.01$ & $<.01$ & $<.01$ & $<.01$ \\
\hline 1,4-Dichlorobenzene & $<.01$ & $<.01$ & $<.01$ & $<.01$ & $<.01$ & $<.01$ & $<.01$ \\
\hline$N, N$-Diethyl-meta-toluamide & .09 & .08 & .09 & .07 & $<.01$ & $<.01$ & .02 \\
\hline 4-Ethylphenol & $<.01$ & $<.01$ & $<.01$ & $<.01$ & $<.01$ & $<.01$ & $<.01$ \\
\hline Hexahydrohexamethylcyclopentabenzopyran & .01 & .02 & $<.01$ & .02 & $<.01$ & $<.01$ & $<.01$ \\
\hline 5-Methyl-1H-benzotriazole & $<.01$ & $<.01$ & $<.01$ & $<.01$ & $<.01$ & $<.01$ & $<.01$ \\
\hline 4-Methylphenol & $<.01$ & $<.01$ & .02 & .01 & $<.01$ & $<.01$ & $<.01$ \\
\hline 4-Nonylphenol & .06 & .05 & $<.05$ & $<.05$ & $<.05$ & $<.05$ & $<.05$ \\
\hline 4-Nonylphenolmonoethoxylate & .11 & .08 & $<.05$ & $<.05$ & $<.05$ & $<.05$ & $<.05$ \\
\hline 4-Nonylphenoldiethoxylate & $<.05$ & $<.05$ & $<.05$ & $<.05$ & $<.05$ & $<.05$ & $<.05$ \\
\hline 4-normal-Octylphenol & $<.01$ & $<.01$ & $<.01$ & $<.01$ & $<.01$ & $<.01$ & $<.01$ \\
\hline 4-tert-Octylphenol & .09 & .06 & .05 & .03 & $<.01$ & $<.01$ & $<.01$ \\
\hline 4-tert-Octylphenolmonoethoxylate & $<.01$ & $<.01$ & $<.01$ & $<.01$ & $<.01$ & $<.01$ & $<.01$ \\
\hline 4-tert-Octylphenoldiethoxylate & $<.01$ & $<.01$ & $<.01$ & $<.01$ & $<.01$ & $<.01$ & $<.01$ \\
\hline 4-tert-Octylphenoltriethoxylate & $<.01$ & $<.01$ & $<.01$ & $<.01$ & $<.01$ & $<.01$ & $<.01$ \\
\hline 4-tert-Octylphenoltetraethoxylate & $<.01$ & $<.01$ & $<.01$ & $<.01$ & $<.01$ & $<.01$ & $<.01$ \\
\hline 4-tert-Octylphenolpentaethoxylate & $<.01$ & $<.01$ & $<.01$ & $<.01$ & $<.01$ & $<.01$ & $<.01$ \\
\hline 4-tert-Pentylphenol & $<.01$ & $<.01$ & $<.01$ & $<.01$ & $<.01$ & $<.01$ & $<.01$ \\
\hline 4-Propylphenol & $<.01$ & $<.01$ & $<.01$ & $<.01$ & $<.01$ & $<.01$ & $<.01$ \\
\hline Triclosan & $<.01$ & $<.01$ & $<.01$ & $<.01$ & $<.01$ & $<.01$ & $<.01$ \\
\hline \multicolumn{8}{|l|}{ Surrogate Recovery (percent) } \\
\hline d6-Bisphenol A & 19 & 40 & 44 & 40 & 39 & 40 & 41 \\
\hline d21-2,6-Di-tert-butyl-4-methylphenol & 20 & 31 & 21 & 38 & 57 & 56 & 49 \\
\hline 4-normal-Nonylphenol & 26 & 47 & 37 & 45 & 36 & 43 & 43 \\
\hline 4-normal-Nonylphenolmonoethoxylate & 52 & 53 & 38 & 43 & 35 & 39 & 39 \\
\hline 4-normal-Nonylphenoldiethoxylate & 44 & 53 & 47 & 53 & 43 & 49 & 49 \\
\hline
\end{tabular}


Table 32. Summary of steroid and steroidal hormone compound data for water samples collected from four microhabitat sites in Sullivan Lake, Minn., on July 8, 2010.

[Microhabitats A and B, residential/septic influenced; microhabitat C, stormwater/boat-ramp influenced; microhabitat D, agriculture influenced; $\mu \mathrm{g} / \mathrm{L}$, microgram per liter; <, less than; Dup, duplicate sample; MS, matrix spike recovery in percent; NQ, not quantifiable]

\begin{tabular}{|c|c|c|c|c|c|c|c|c|c|c|c|c|}
\hline Compound & $\begin{array}{c}\text { Site } \\
\text { A } \\
\mu \mathrm{g} / \mathrm{L}\end{array}$ & $\begin{array}{l}\text { Site } \\
\text { A-Dup } \\
\mu g / L\end{array}$ & $\begin{array}{c}\text { Site } \\
\text { A-MS } \\
\text { percent }\end{array}$ & $\begin{array}{c}\text { Site } \\
\text { B } \\
\mu \mathrm{g} / \mathrm{L}\end{array}$ & $\begin{array}{l}\text { Site } \\
\text { B-Dup } \\
\mu \mathrm{g} / \mathrm{L}\end{array}$ & $\begin{array}{c}\text { Site } \\
\text { B-MS } \\
\text { percent }\end{array}$ & $\begin{array}{c}\text { Site } \\
\text { C } \\
\mu \mathrm{g} / \mathrm{L}\end{array}$ & $\begin{array}{l}\text { Site } \\
\text { C-Dup } \\
\mu g / L\end{array}$ & $\begin{array}{c}\text { Site } \\
\text { C-MS } \\
\text { percent }\end{array}$ & $\begin{array}{c}\text { Site } \\
\text { D } \\
\mu \mathrm{g} / \mathrm{L}\end{array}$ & $\begin{array}{l}\text { Site } \\
\text { D-Dup } \\
\mu \mathrm{g} / \mathrm{L}\end{array}$ & $\begin{array}{c}\text { Site } \\
\text { D-MS } \\
\text { percent }\end{array}$ \\
\hline 4-Androstene-3,17-dione & $<0.0001$ & $<0.0001$ & 138 & 0.0009 & 0.0005 & 118 & 0.0005 & 0.0005 & 134 & 0.0014 & 0.0012 & 133 \\
\hline cis-Androsterone & $<.0001$ & $<.0001$ & 150 & $<.0001$ & $<.0001$ & 139 & $<.0001$ & $<.0001$ & 130 & $<.0001$ & $<.0001$ & 150 \\
\hline Cholesterol & 5.7 & NQ & 348 & 7.6 & 4.4 & 266 & 30 & 11 & -664 & 23 & NQ & 327 \\
\hline Coprostanol & .029 & .065 & 104 & .042 & .025 & 103 & .053 & .090 & 86 & .20 & .97 & 89 \\
\hline Diethylstilbestrol & $<.0001$ & $<.0001$ & 97 & $<.0001$ & $<.0001$ & 66 & $<.0001$ & $<.0001$ & 97 & $<.0001$ & $<.0001$ & 97 \\
\hline Equilenin & $<.0001$ & $<.0001$ & 105 & $<.0001$ & $<.0001$ & 86 & $<.0001$ & $<.0001$ & 101 & $<.0001$ & $<.0001$ & 107 \\
\hline Equilin & $<.0001$ & $<.0001$ & 98 & $<.0001$ & $<.0001$ & 78 & $<.0001$ & $<.0001$ & 97 & $<.0001$ & $<.0001$ & 76 \\
\hline $17 \alpha$-Estradiol & $<.0001$ & $<.0001$ & 117 & $<.0001$ & $<.0001$ & 132 & $<.0001$ & $<.0001$ & 112 & $<.0001$ & $<.0001$ & 119 \\
\hline $17 \beta$-Estradiol & $<.0001$ & $<.0001$ & 107 & $<.0001$ & $<.0001$ & 106 & $<.0001$ & $<.0001$ & 108 & $<.0001$ & $<.0001$ & 111 \\
\hline Estriol & $<.0001$ & $<.0001$ & 115 & $<.0001$ & $<.0001$ & 73 & $<.0001$ & $<.0001$ & 62 & $<.0001$ & $<.0001$ & 235 \\
\hline Estrone & .0007 & .0008 & 120 & .0010 & .0009 & 111 & .0011 & .0009 & 110 & .0008 & .0010 & 107 \\
\hline $17 \alpha$-Ethynylestradiol & $<.0001$ & $<.0001$ & 110 & $<.0001$ & $<.0001$ & 100 & $<.0001$ & $<.0001$ & 103 & $<.0001$ & $<.0001$ & 104 \\
\hline Mestranol & $<.0001$ & $<.0001$ & 107 & $<.0001$ & $<.0001$ & 99 & $<.0001$ & $<.0001$ & 106 & $<.0001$ & $<.0001$ & 106 \\
\hline Norethindrone & $<.0001$ & $<.0001$ & 106 & $<.0001$ & $<.0001$ & 99 & $<.0001$ & $<.0001$ & 104 & $<.0001$ & $<.0001$ & 106 \\
\hline Progesterone & $<.0001$ & $<.0001$ & 15 & $<.0001$ & $<.0001$ & 26 & $<.0001$ & $<.0001$ & 27 & $<.0001$ & $<.0001$ & 22 \\
\hline Testosterone & $<.0001$ & $<.0001$ & 124 & $<.0001$ & $<.0001$ & 114 & $<.0001$ & $<.0001$ & 121 & $<.0001$ & $<.0001$ & 124 \\
\hline dihydro-Testosterone & $<.0001$ & $<.0001$ & 130 & $<.0001$ & $<.0001$ & 109 & $<.0001$ & $<.0001$ & 123 & $<.0001$ & $<.0001$ & 113 \\
\hline epi-Testosterone & $<.0001$ & $<.0001$ & 128 & $<.0001$ & $<.0001$ & 121 & $<.0001$ & $<.0001$ & 120 & $<.0001$ & $<.0001$ & 132 \\
\hline 11-keto-Testosterone & $<.0001$ & $<.0001$ & 81 & $<.0001$ & $<.0001$ & 69 & $<.0001$ & $<.0001$ & 95 & $<.0001$ & $<.0001$ & 91 \\
\hline \multicolumn{13}{|c|}{ Surrogate Recovery (percent) } \\
\hline d7-Cholesterol & 47 & $<10$ & & 37 & 41 & & 19 & 10 & & 40 & $<10$ & \\
\hline d12-Chrysene & 105 & 106 & & 183 & 115 & & 115 & 119 & & 105 & 104 & \\
\hline d8-Diethylstibestrol & 59 & $<10$ & & 47 & 18 & & 42 & 18 & & 34 & $<10$ & \\
\hline${ }^{13} \mathrm{C}_{6}$-Estradiol & 80 & 18 & & 56 & 33 & & 36 & 28 & & 28 & $<10$ & \\
\hline $\mathrm{d} 2$-epi-Estriol & 42 & 18 & & 46 & 49 & & 39 & 19 & & 16 & 18 & \\
\hline${ }^{13} \mathrm{C}_{6}$-Estrone & 96 & 30 & & 75 & 97 & & 71 & 65 & & 97 & 31 & \\
\hline d4-Ethynylestradiol & 82 & 26 & & 62 & 71 & & 47 & 49 & & 70 & 25 & \\
\hline d3-Medroxyprogesterone & 19 & 12 & & 45 & 33 & & 44 & 35 & & 28 & 21 & \\
\hline d4-Mestranol & 83 & 30 & & 63 & 70 & & 48 & 48 & & 71 & 28 & \\
\hline d3-Nandrolone & 63 & 24 & & 54 & 61 & & 54 & 45 & & 30 & 17 & \\
\hline
\end{tabular}


Table 33. Summary of steroid and steroidal hormone compound data for water samples collected from four microhabitat sites in Sullivan Lake, Minn., on October $12,2010$.

[Microhabitats A and B, residential/septic influenced; microhabitat C, stormwater/boat-ramp influenced; microhabitat D, agriculture influenced; $\mu \mathrm{g} / \mathrm{L}$, microgram per liter; <, less than]

\begin{tabular}{|c|c|c|c|c|}
\hline Compound & $\begin{array}{c}\text { Site } \\
\text { A } \\
\mu \mathrm{g} / \mathrm{L}\end{array}$ & $\begin{array}{c}\text { Site } \\
\text { B } \\
\mu g / L\end{array}$ & $\begin{array}{c}\text { Site } \\
\text { C } \\
\mu \mathrm{g} / \mathrm{L}\end{array}$ & $\begin{array}{c}\text { Site } \\
\text { D } \\
\mu \mathrm{g} / \mathrm{L}\end{array}$ \\
\hline 4-Androstene-3,17-dione & $<0.0001$ & $<0.0001$ & $<0.0001$ & $<0.0001$ \\
\hline cis-Androsterone & $<.0001$ & $<.0001$ & $<.0001$ & $<.0001$ \\
\hline Cholesterol & 3.3 & 3.4 & 2.8 & 3.2 \\
\hline Coprostanol & .042 & .017 & .017 & .030 \\
\hline Diethylstilbestrol & $<.0001$ & $<.0001$ & $<.0001$ & $<.0001$ \\
\hline Equilenin & $<.0001$ & $<.0001$ & $<.0001$ & $<.0001$ \\
\hline Equilin & $<.0001$ & $<.0001$ & $<.0001$ & $<.0001$ \\
\hline $17 \alpha$-Estradiol & $<.0001$ & $<.0001$ & $<.0001$ & $<.0001$ \\
\hline $17 \beta$-Estradiol & $<.0001$ & $<.0001$ & $<.0001$ & $<.0001$ \\
\hline Estriol & $<.0001$ & $<.0001$ & $<.0001$ & $<.0001$ \\
\hline Estrone & $<.0001$ & $<.0001$ & $<.0001$ & .0006 \\
\hline $17 \alpha$-Ethynylestradiol & $<.0001$ & $<.0001$ & $<.0001$ & $<.0001$ \\
\hline Mestranol & $<.0001$ & $<.0001$ & $<.0001$ & $<.0001$ \\
\hline Norethindrone & $<.0001$ & $<.0001$ & $<.0001$ & $<.0001$ \\
\hline Progesterone & $<.0001$ & $<.0001$ & $<.0001$ & $<.0001$ \\
\hline Testosterone & $<.0001$ & $<.0001$ & $<.0001$ & $<.0001$ \\
\hline dihydro-Testosterone & $<.0001$ & $<.0001$ & $<.0001$ & $<.0001$ \\
\hline epi-Testosterone & $<.0001$ & $<.0001$ & $<.0001$ & $<.0001$ \\
\hline 11-keto-Testosterone & $<.0001$ & $<.0001$ & $<.0001$ & $<.0001$ \\
\hline \multicolumn{5}{|c|}{ Surrogate Recovery (percent) } \\
\hline d7-Cholesterol & 39 & 46 & 53 & 15 \\
\hline d12-Chrysene & 82 & 94 & 94 & 104 \\
\hline d8-Diethylstibestrol & 80 & 69 & 83 & 52 \\
\hline${ }^{13} \mathrm{C}_{6}$-Estradiol & 63 & 65 & 58 & 50 \\
\hline d2-epi-Estriol & 85 & 98 & 97 & 72 \\
\hline${ }^{13} \mathrm{C}_{6}$-Estrone & 118 & 116 & 106 & 81 \\
\hline d4-Ethynylestradiol & 91 & 99 & 92 & 75 \\
\hline d3-Medroxyprogesterone & 55 & 43 & 47 & 42 \\
\hline d4-Mestranol & 103 & 101 & 94 & 74 \\
\hline d3-Nandrolone & 121 & 96 & 98 & 74 \\
\hline
\end{tabular}

